\title{
STRUCTURAL DAMAGE ASSESSMENT USING ARTIFICIAL NEURAL NETWORKS AND ARTIFICIAL IMMUNE SYSTEMS
}

\author{
A Thesis \\ presented to \\ the Faculty of California Polytechnic State University, \\ San Luis Obispo
}

\author{
In Partial Fulfillment \\ of the Requirements for the Degree \\ Master of Science in Electrical Engineering
}

by

Arthur Q.X. Shi

December 2015 
(C) 2015

Arthur Q.X. Shi

ALL RIGHTS RESERVED 
TITLE:

AUTHOR:

DATE SUBMITTED:

COMMITTE CHAIR: Xiao-Hua (Helen) Yu, Ph.D.

Professor of Electrical Engineering

COMMITTEE MEMBER: Xiaomin Jin, Ph.D.

Professor of Electrical Engineering

COMMITTEE MEMBER: David Janzen, Ph.D.

Professor of Computer Science 


\begin{abstract}
Structural Damage Assessment Using Artificial Neural Networks
\end{abstract}

and Artificial Immune Systems

Arthur Q.X. Shi.

Structural health monitoring (SHM) systems have been technologically advancing over the past few years. Improvements in fabrication and microelectronics allow the development of highly sophisticated sensor arrays, capable of detecting and transmitting an unprecedented amount of data. As the complexity of the hardware increases, research has been performed in developing the means to best utilize and effectively process the data. Algorithms from other computational fields are being introduced for the first time into SHM systems. Amongst them, the artificial neural network (ANN) and artificial immune systems (AIS) show great potential. In this thesis, features are extracted out of the acceleration data with the use of discrete wavelet transforms (DWT)s first. The DWT coefficients are used to calculate energy ratios, which are then classified using a neural network and an AIS algorithm known as negative selection (NS). The effectiveness of both methods are validated using simulated acceleration data of a four story structure exhibiting various damage states via computer simulation.

Keywords: Structural Health Monitoring, Wavelet Decomposition, Artificial Neural Network, Artificial Immune System, Negative Selection 


\section{ACKNOWLEDGMENTS}

The completion of this thesis would be impossible without the support of many family and friends.

I would like to thank my thesis advisor Dr. Xiao-Hua (Helen) Yu for her continuous guidance, patience, and support through the extended endeavor. Her confidence and technical expertise in the field of computational intelligence helped guide this thesis to the end.

To my thesis board member Xiaomin Jin, thank you for your flexibility and willingness to be on my defense board at such short notice. Thank you David Janzen, for all of your wisdom, guidance, encouragement, and firm words.

I would like to thank my parents for their steadfast support and love. Their patience and understanding stretches beyond what I deserve. I would not be here without them.

To my friend Mark, who has offered everything he has in order to help me progress this thesis, thank you. You are an amazing friend.

To my fiancée Kristina, thank you for your love and your steadfast expression of it through daily life. You have been so patient and supportive through all these years. I love you.

To the Lord and Maker of the universe, who strengthened me in ways no one else could, who authored the laws of physics and redeemed my life for eternity, may all the glory that's due to You be given. 


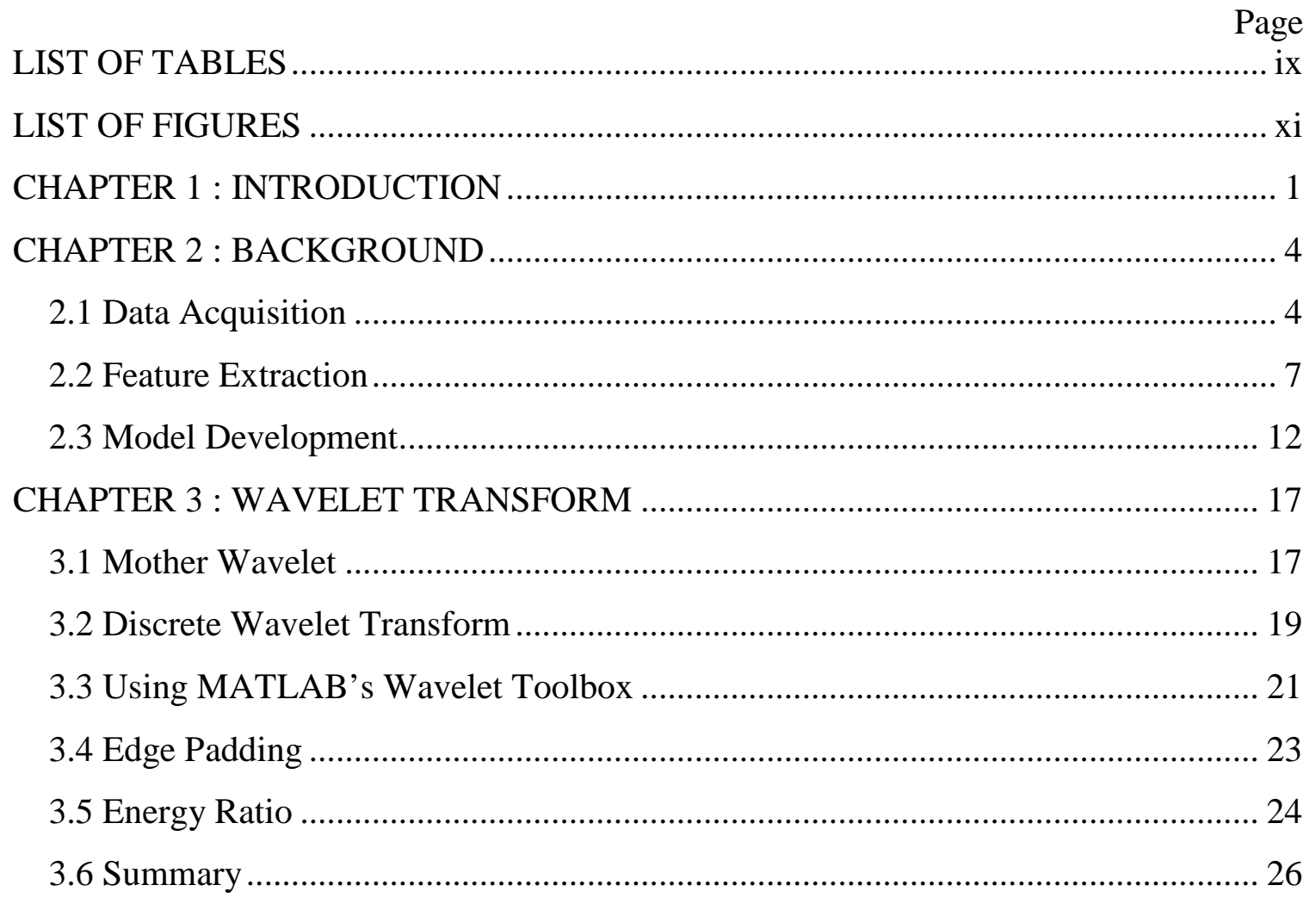

CHAPTER 4 : ARTIFICIAL NEURAL NETWORKS................................................... 27

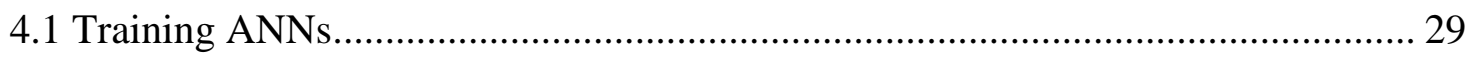

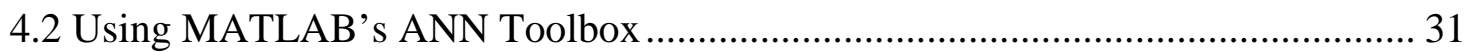

4.3 Number of Neurons and Hidden Layers .............................................................. 32

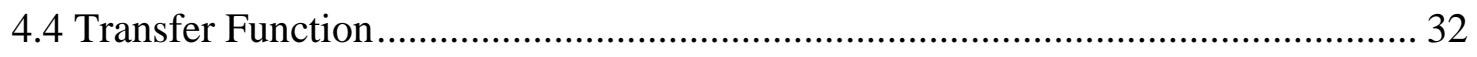

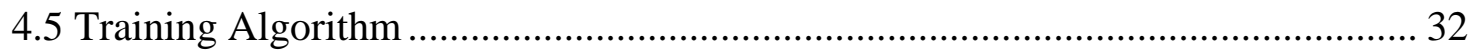

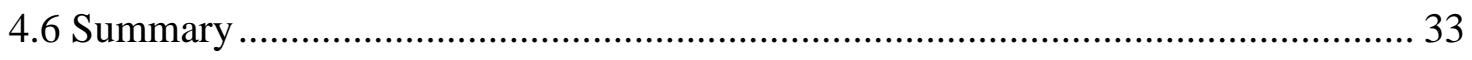

CHAPTER 5 : ARTIFICIAL IMMUNE SYSTEM...................................................... 35

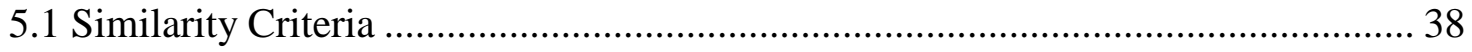

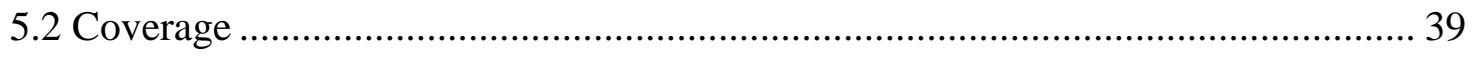

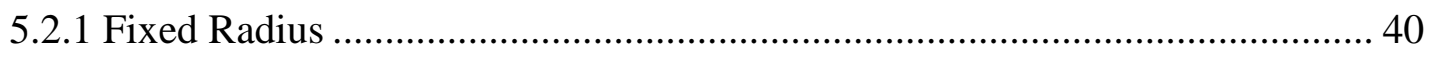

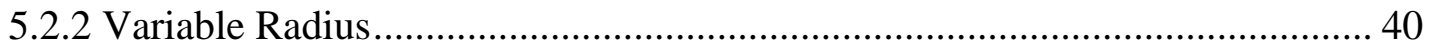

5.2.3 Overlapping Radii ........................................................................................ 42

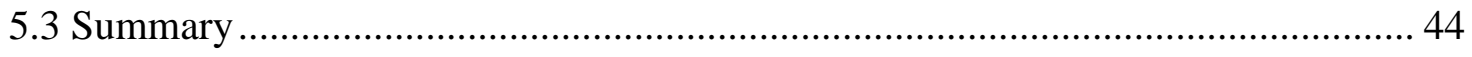

CHAPTER 6 : DATAGEN ..................................................................................... 45 


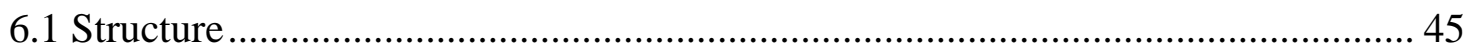

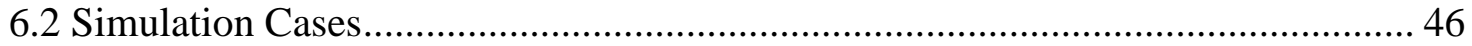

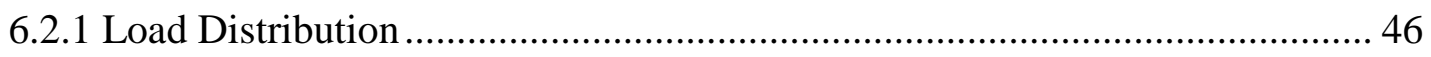

6.2.2 Load Symmetry............................................................................................. 47

6.2.3 External Excitation..................................................................................... 47

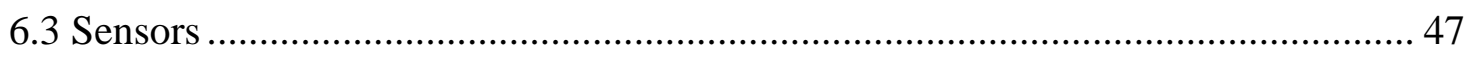

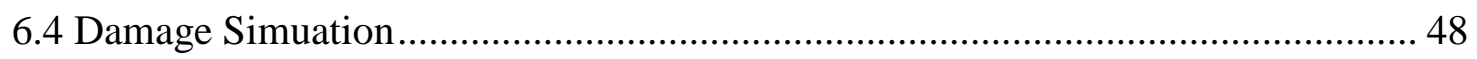

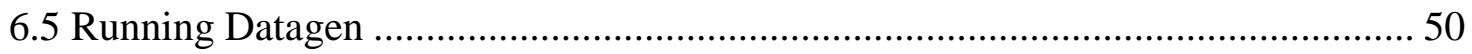

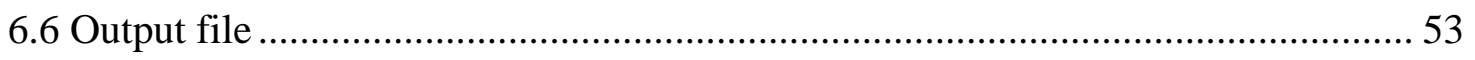

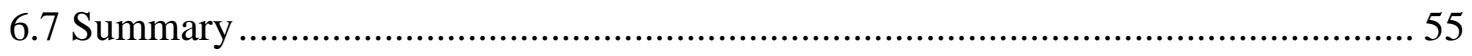

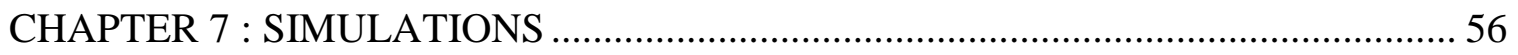

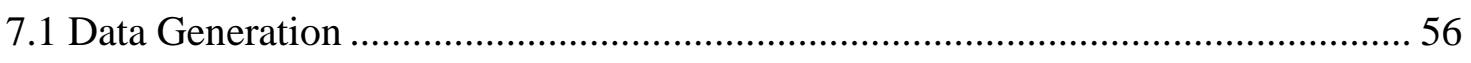

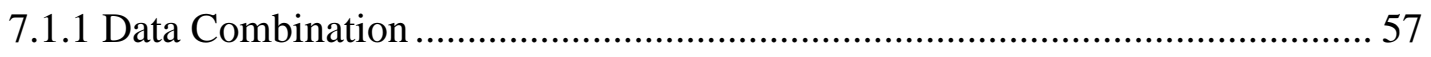

7.1.2 Damage Simulation...................................................................................... 58

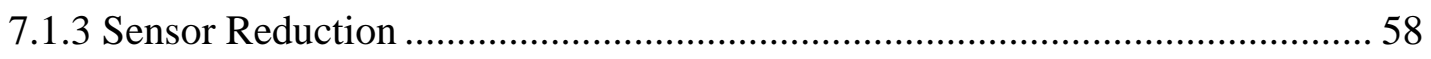

7.1.4 Damage Matrix Parsing ................................................................................. 59

7.1.5 Data Generation Output Summary .................................................................. 60

7.2 Feature Extraction Using Wavelet Transforms....................................................... 60

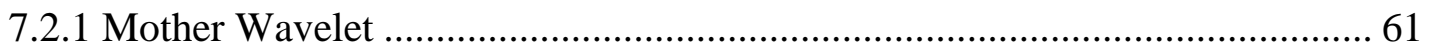

7.2.2 Decomposition Level...................................................................................... 61

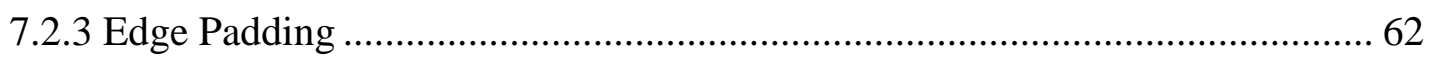

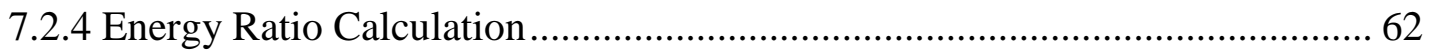

7.2.5 Feature Extraction Output Summary ………………………………………..... 63

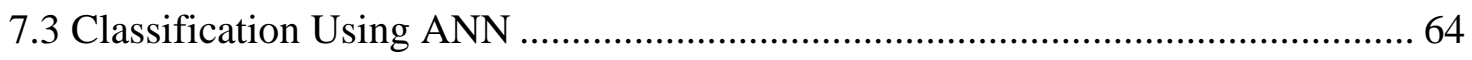

7.3.1 Approach One- Boolean System........................................................................ 65

7.3.2 Approach Two- Multi-damage System........................................................... 71

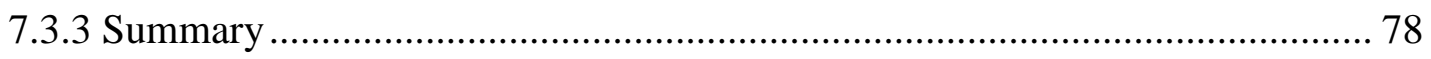

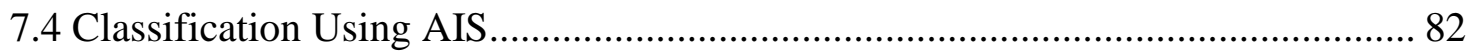

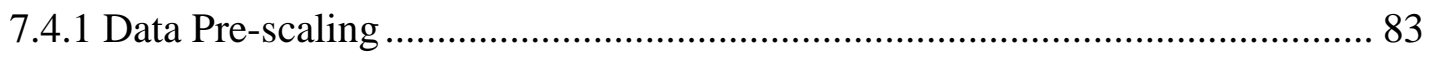

7.4.2 Preliminary Optimization................................................................................. 83

7.4.3 Approach One- Full Sensor Data ....................................................................... 84 


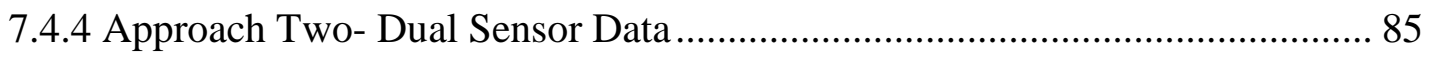

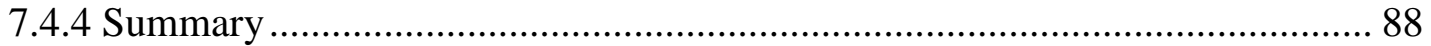

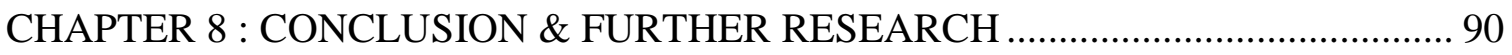

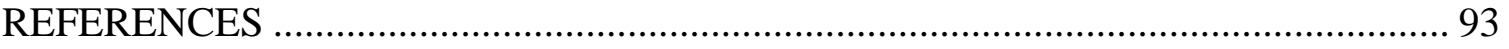

APPENDICES

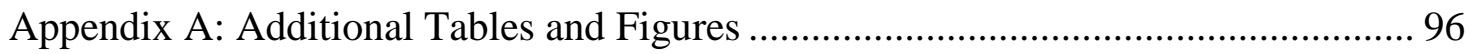

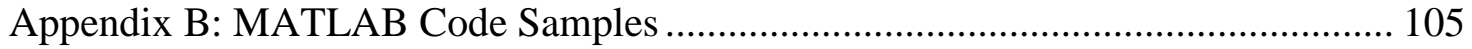




\section{LIST OF TABLES}

Table

Table 2.1: Biological-Artificial immune system equivalent examples [22] .................... 15

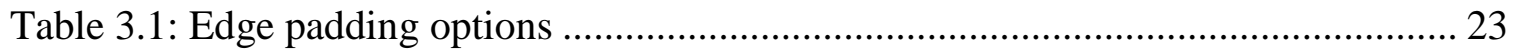

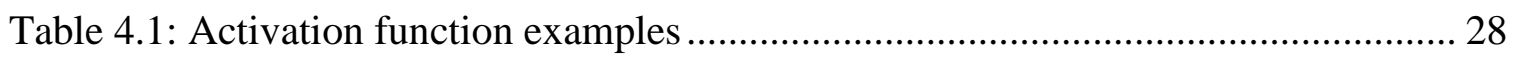

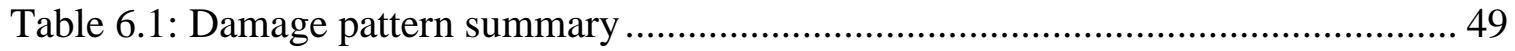

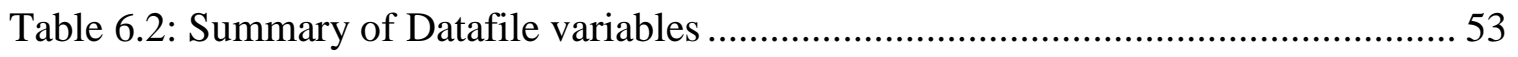

Table 7.1: Data generation setup parameters in summary .............................................. 57

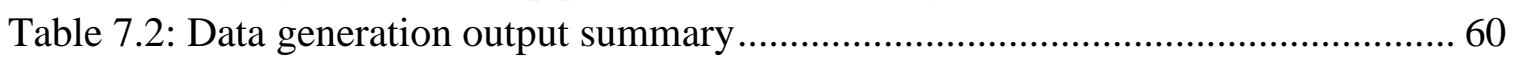

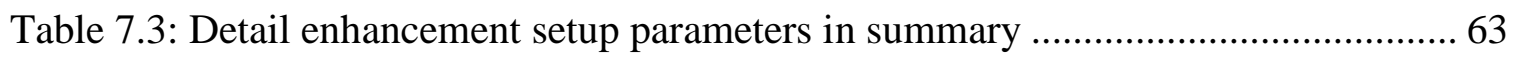

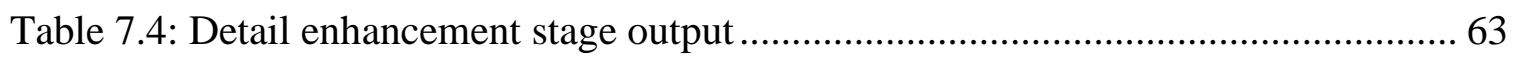

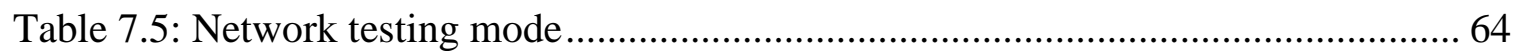

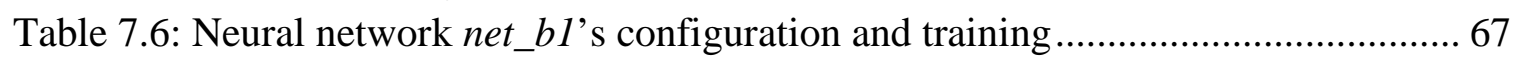

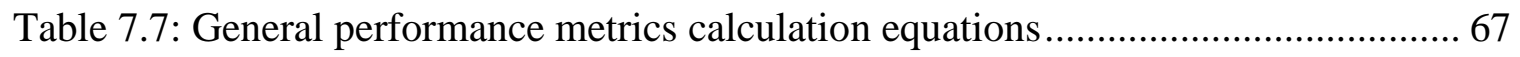

Table 7.8: Neural network net_b1 [4,60,30,1] transient results..................................... 68

Table 7.9: Neural network net_b1 [4,60,30,1] transient summary .................................. 68

Table 7.10: Neural network net_b1 [4,60,30,1] steady damage results........................... 68

Table 7.11: Neural network net_b1 [4,60,30,1] steady damage summary ....................... 68

Table 7.12: Neural network net_b2 [4,60,30,1] transient results.................................... 69

Table 7.13: Neural network net_b2 [4,60,30,1] transient summary ................................ 69

Table 7.14: Neural network net_b2 [4,60,30,1] steady damage results.......................... 69

Table 7.15: Neural network net_b2 [4,60,30,1] steady damage summary ...................... 69

Table 7.16: Neural network net_b1a's setup parameters............................................. 70

Table 7.17: Neural network net_b1a $[4,30,60,60,1]$ transient results............................... 71

Table 7.18: Neural network net_b1a [4,30,60,60,1] transient summary ......................... 71

Table 7.19: Neural network net_b1a [4,30,60,60,1] steady damage results.................... 71

Table 7.20: Neural network net_b1a [4,30,60,60,1] steady damage summary ............... 71

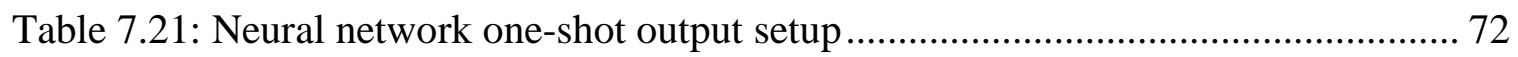

Table 7.22: Neural network net_1shot_1's setup parameters ........................................ 73

Table 7.23: Neural network net_1shot_1 [4,80,60,9] transient results............................ 74

Table 7.24: Neural network net_1shot_1 [4,80,60,9] transient summary ....................... 75

Table 7.25: Neural network net_1shot_1 $[4,80,60,9]$ steady damage results ................... 74

Table 7.26: Neural network net_1shot_1 [4,80,60,9] steady damage summary.............. 75

Table 7.27: Neural network net_1shot_2 [4,80,60,9] transient results............................ 76

Table 7.28: Neural network net_1shot_2 [4,80,60,9] transient summary ......................... 76

Table 7.29: Neural network net_1shot_2 [4,80,60,9] steady damage results ................... 76

Table 7.30: Neural network net_1shot_2 [4,80,60,9] steady damage summary............... 76

Table 7.31: Neural network net_1shot_1a [4,80,60,9] transient results.......................... 78

Table 7.32: Neural network net_1shot_1a [4,80,60,9] transient summary ..................... 78

Table 7.33: Neural network net_1shot_1a $[4,80,60,9]$ steady damage results ................ 78 
Table 7.34: Neural network net_1shot_1a [4,80,60,9] steady damage summary............. 78

Table 7.35: Boolean network average results for native category data .......................... 79

Table 7.36: Boolean network average results for non-native category data .................... 79

Table 7.37: Multi-damage network average results for native category data.................. 80

Table 7.38: Multi-damage network average results for non-native category data ........... 81

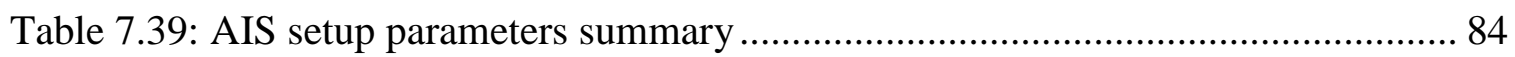

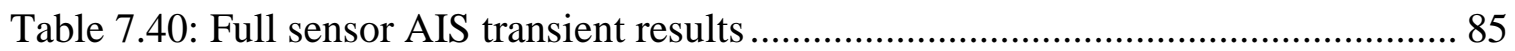

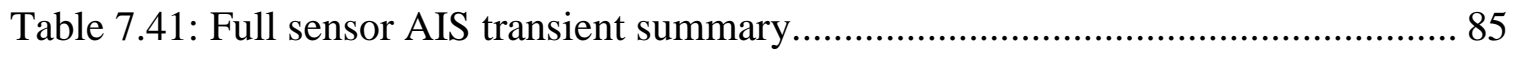

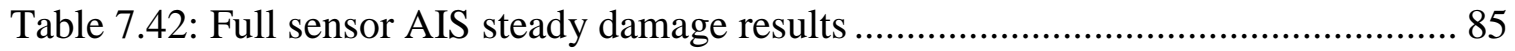

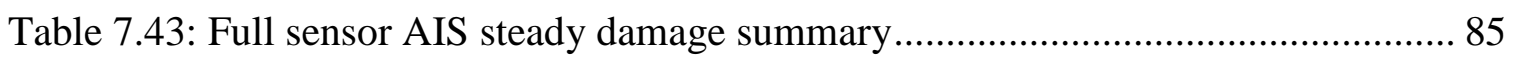

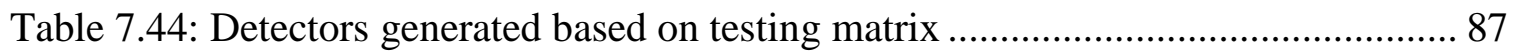

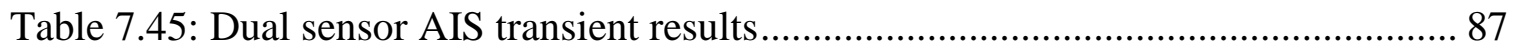

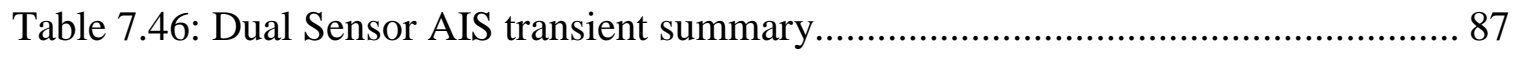

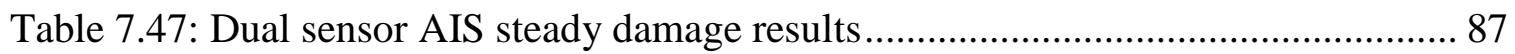

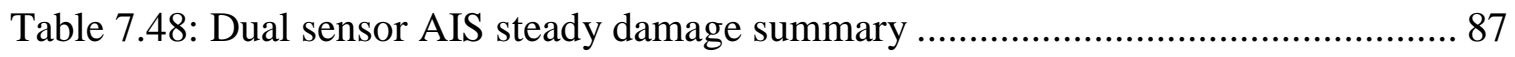

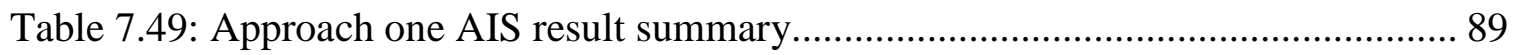

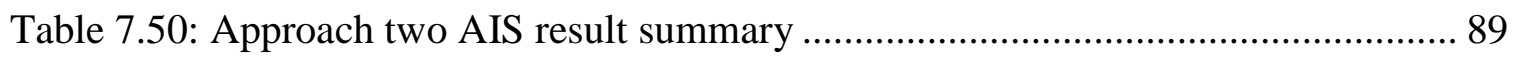

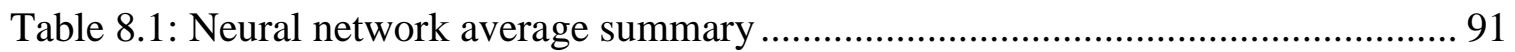

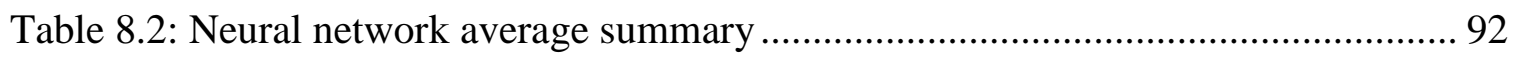

Table A.1: Neural network net_1shot_1's transient damage confusion matrix................ 99

Table A.2: Neural network net_1shot_1's steady damage confusion matrix .................. 99

Table A.3: Neural network net_1shot_2's transient damage confusion matrix............... 100

Table A.4: Neural network net_1shot_2's steady damage confusion matrix ................. 100

Table A.5: Neural network net_1shot_1a's transient damage confusion matrix............. 101

Table A.6: Neural network net_1shot_1a's steady damage confusion matrix ................ 101

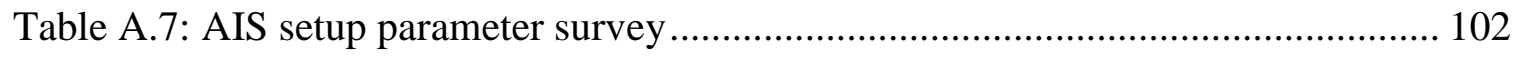

Table A.8: Dual sensor (2\&3) AIS transient damage results........................................ 103

Table A.9: Dual Sensor (2\&3) AIS transient damage summary .................................. 103

Table A.10: Dual sensor (2\&3) AIS steady ............................................................ 103

Table A.11: Dual Sensor (2\&3) AIS steady damage summary ................................... 103 


\section{LIST OF FIGURES}

Figure

Figure 2.1: Spectral shift due to damage [8] ............................................................ 5

Figure 2.2: Level 1 decomposition coefficients showing spikes at damage [14] .............. 8

Figure 2.3: Level 3 decomposition coefficients showing spike at damage [15]................ 8

Figure 2.4: Decomposition of a chattering lathe [16] ................................................. 10

Figure 2.5: Level 1 detail coefficients showing spike at 5 second mark [18] .................. 11

Figure 2.6: "Signatures" of various damage patterns (D0-D6) [12] ................................ 12

Figure 2.7: Spring-mass models: (a) 3 DOF model. (b) Kabe’s 8 DOF model [20] ........ 14

Figure 2.8: Flowchart of antigen creation and storage [22] .......................................... 16

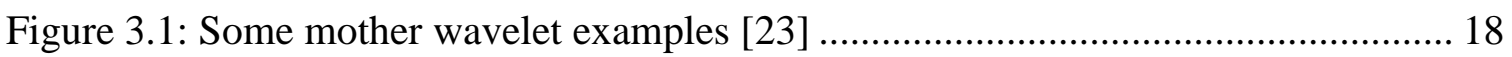

Figure 3.2: Wavelet decomposition coefficient structure overview ............................... 20

Figure 3.3: Sensor data decomposition example ..................................................... 21

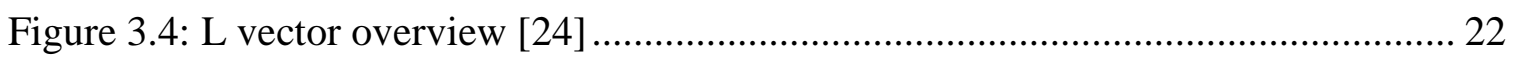

Figure 3.5: cA7 coefficients with default 'sym' padding. Both edges distorted. ............. 24

Figure 3.6: cA7 coefficients with 'zpd' padding. No edge distortion ............................. 24

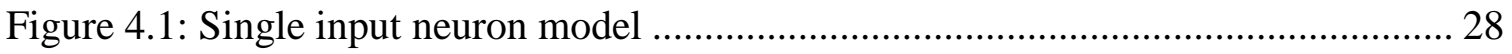

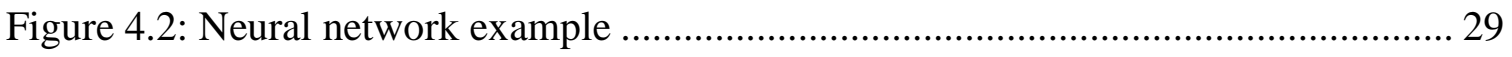

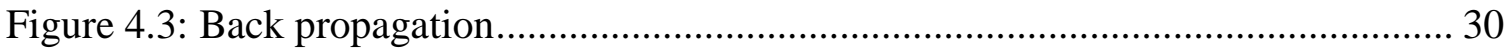

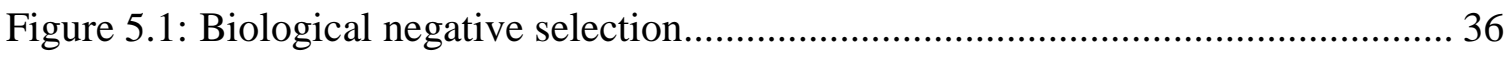

Figure 5.2: Artificial negative selection algorithm ................................................. 37

Figure 5.3: (a) NS algorithm fixed radius training and (b) testing ................................ 38

Figure 5.4: Variable radius detector generation example ............................................ 41

Figure 5.5: (a) NS variable radii training and (b) testing.............................................. 42

Figure 5.6: (a) Variable radii, overlapping detectors training and (b) testing ................. 43

Figure 6.1: Datagen's physical structure model .......................................................... 46

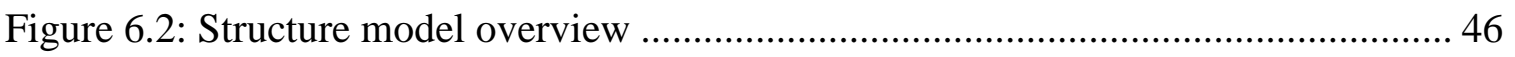

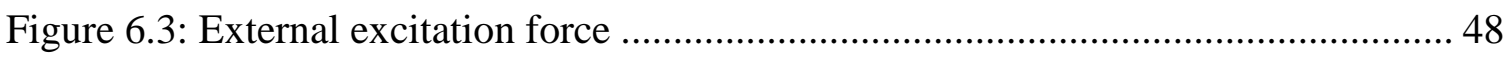

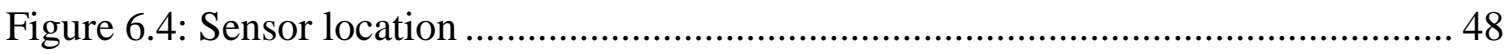

Figure 6.5: Overview of Datagen's GUI menus...................................................... 50

Figure 6.6: Visualized acceleration data for damage case 2 ........................................ 54

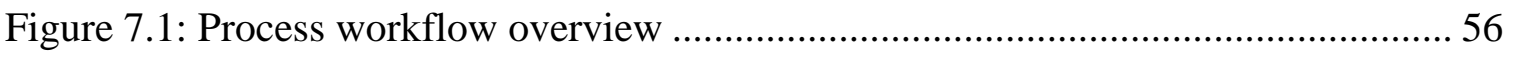

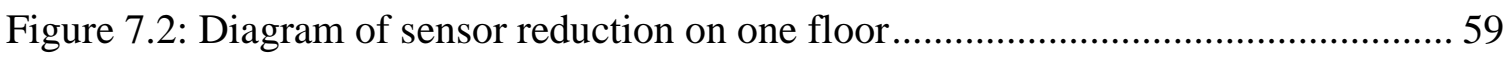

Figure 7.3: Single 1.5 second simulation run parsing example ..................................... 60

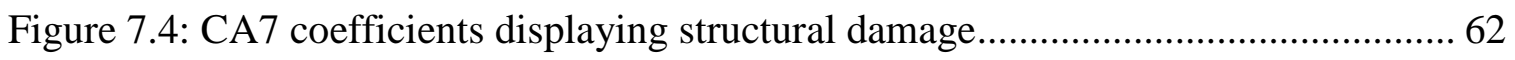

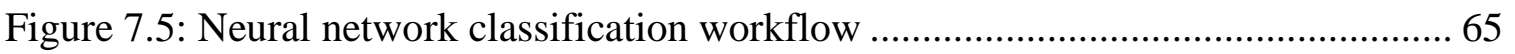

Figure A.1: DWT using 'Haar' wavelet on sensor \#2, damage pattern \#7 ...................... 96

Figure A.2: DWT using 'db2' wavelet on sensor \#2, damage pattern \#7 ....................... 96

Figure A.3: Neural network net_b1's training MSE curve........................................... 97

Figure A.4: Neural network net_b2’s training MSE curve .......................................... 97 
Figure A.5: Neural network net_b1a's training MSE curve.......................................... 98

Figure A.6: Neural network net_1shot_1's training MSE curve ................................... 99

Figure A.7: Neural network net_1shot_2's training MSE curve ................................... 100

Figure A.8: Neural network net_1shot_1a's training MSE curve ................................ 101

Figure A.9: AIS visualization of damage pattern 2, (b) zoomed in at [0 0.1] [0 0.1] .... 104

Figure A.10: AIS visualization of damage pattern 7, (b) zoomed in at [0 0.1] [0 0.1]... 104 


\section{CHAPTER 1 : INTRODUCTION}

Humans have been building large civil structures for thousands of years. These constructs are prone to damage due to various factors such as normal wear and tear, excessive stress, or environmental maladies. Continual applications of these stresses contribute to the loss of structural integrity. Eventually, the structure becomes unsafe to use and must be repaired or condemned for destruction.

In response to the inevitable wear of structures, the development of Structural Health Monitoring systems has become more important. The task of determining the health of a structure has been the subject of research for many decades. As safety standards improve, an accurate analysis of a building's integrity is necessary to ensure timely maintenance, preventing catastrophes. As larger, more complex, high-importance buildings are constructed, the value of nondestructive evaluation (NDE) methods rises. This is classically accomplished by visual inspections of the building in question. However, many structural failures may either be hidden or missed by the naked eye. Thus, modern SHM methods must be developed to detect, evaluate, and asses the health of a building.

Current SHM systems normally involve two components: a network of sensors which collects performance data, and an analysis algorithm which interprets the collected data into actual structural health. As technologies improve, sensors become increasingly cost-effective while boasting greater sensitivities and higher sampling rates. This leads to a wider bandwidth of sensory data, potentially giving a better situational awareness of the structure's integrity. However, this data must be further processed by the SHM system in order to produce conclusions pertaining to the health of the building. 
There are multiple approaches to analyzing the collected sensor data. Traditionally, this is accomplished by matching the collected data to a set of pre-defined criteria based on structural analysis. This often requires a thorough understanding of the structure and the development of a very accurate mathematical model for it. Artificial neural networks (ANNs) have proven to effectively model non-linear systems which would otherwise be difficult to characterize using conventional means. The negative selection (NS) algorithm, which is a sub-category of artificial immune system (AIS) algorithms, offers strong heuristic abilities, allowing it to effectively detect "abnormal" entities while requiring little "normal" data. These properties make the ANN and NS algorithms strong candidates for SHM systems.

This thesis presents a novel method for analyzing structural data. A discrete wavelet transform (DWT) is used to pre-process the acceleration response. The energy ratio is then calculated using the wavelet coefficients. The results are passed to an artificial neural network (ANN) or negative selection (NS) algorithm in order to determine the fault-status of a structure. The effectiveness of each method is then tested using a benchmark program created by the American Society of Civil Engineers (ASCE) called Datagen.

This study is separated into seven chapters. Chapter 2 provides a literature review of current research into each SHM section. Chapter 3 overviews the background concepts on discrete wavelet transforms. Chapter 4 provides a rundown of artificial neural networks. Chapter 5 covers the basics of AIS algorithms, specifically the negative selection algorithm. Chapter 6 describes ASCE’s finite-element model Datagen. It covers in detail the structure of the model, how sensor data is generated, as well as describing 
the fault types it generates. Chapter 7 covers the datasets, testing methodology, simulation outcomes, and results summary from this research. Finally, chapter 8 presents the conclusion of the findings and potential for further studies. Appendix A includes additional data tables not included in the body of the report. Appendix B includes samples of the actual MATLAB code written. 


\section{CHAPTER 2 : BACKGROUND}

Current Structural Health Monitoring (SHM) systems are dependent upon a combination of Usage Monitoring (UM) and Non-Destructive Evaluation (NDE) methods. In general, this consists of monitoring the structure over a period of time using sensors, recording any damage to the structure, and approximating the health of the structure using statistical analysis [1]. SHM can be described in a three part process: (1) data acquisition, (2) feature extraction, and (3) model development [2].

\subsection{Data Acquisition}

The data acquisition portion of SHM describes how and what relevant data is acquired. Historically, visual inspection is the traditional NDE data acquisition method. Inspectors would observe and keep records of the structure over its life cycle [3] [4]. Advanced tools have been developed to improve this method. [5] is an example of this, showing that a $3 \%$ structural stiffness can be detected using high resolution pictures captured by a CCD (charge coupled device) camera with a resolution of 2048 by 2048 pixels. Visual inspection works well with structures with open and easily accessible infrastructure such as bridges, but the method has several inadequacies. First and foremost, visual inspection requires spatial access to the entire infrastructure. This is often not feasible in modern office buildings where the infrastructure is hidden and inaccessible [6]. Even if certain elements are visible, visual inspection can be time consuming and costly, especially for very large structures. Thus, it would only be accomplished very periodically, or after certain events that tend to damage infrastructure such as seismic activity. 
As technology advances, the trend veers more towards automated data acquisition with the use of electronic sensors. Thomas et al. [7] used sixteen wired PCB accelerometers placed strategically across a girder to successfully detect impact damage of various degrees. Belisario-Briceno et al. [8] placed a combination of conventional and piezoelectric sensors on a reinforced concrete beam. Data from both types of sensors are passed through a discrete Fourier transform with a range of $10-20 \mathrm{kHz}$ and a resolution of 0.33 Hz. Damage was successfully detected by a shift in the spectral levels of the transform (Figure 2.1).

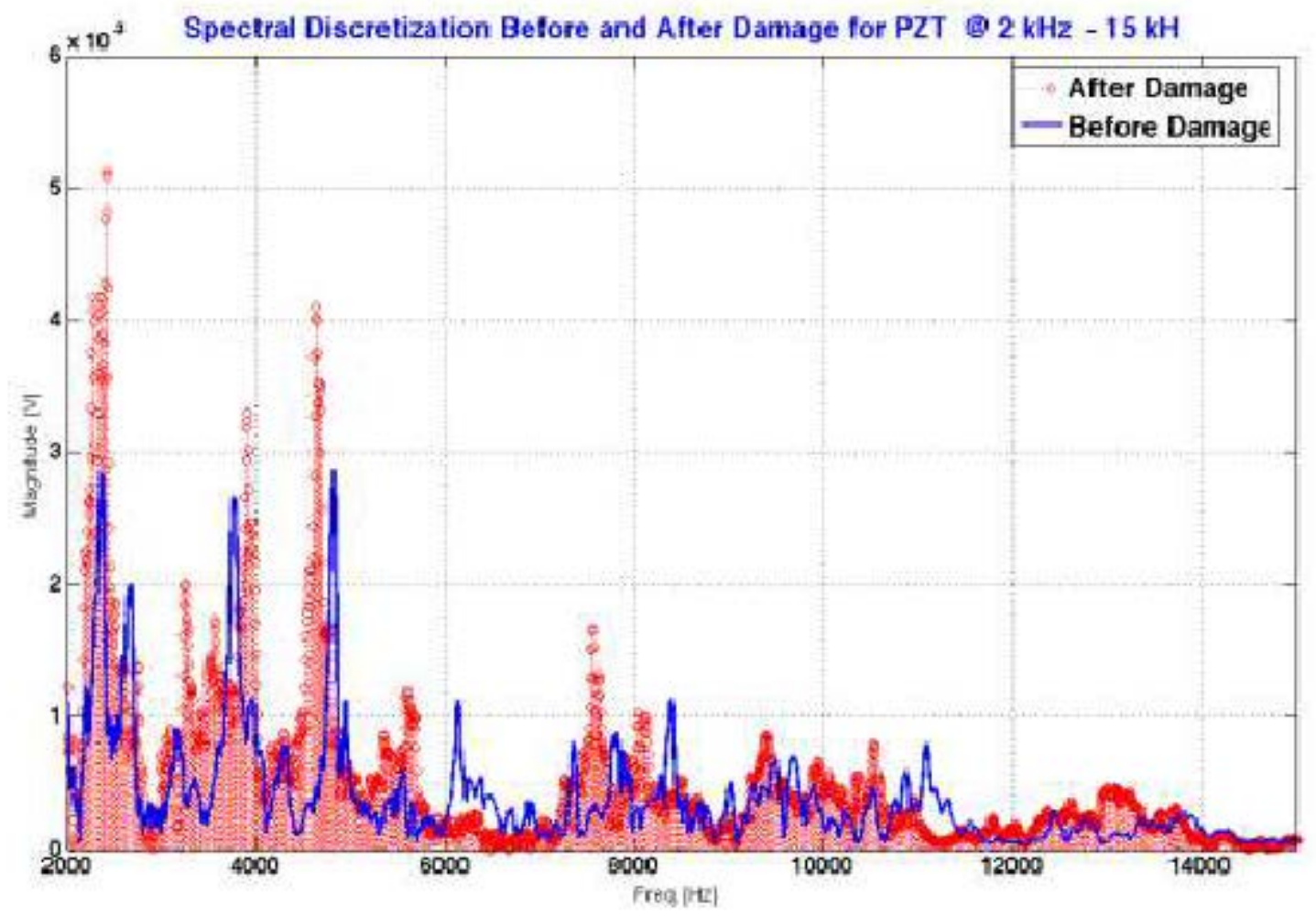

Figure 2.1: Spectral shift due to damage [8] 
In addition, data acquisition also considers what type of data will be recorded. Accelerometers recording acceleration data are the most commonly used sensors; modal analysis is employed as the foundation of modern sensor based SHM methods. This is publicized by Farrar et al. [2], where damage in a spring-mass system was detected and categorized using modal analysis. In addition, Hearn et al. [9] demonstrated the effectiveness of modal analysis in the detection of damage due to fatigue loading on a welded steel frame. Saadat et al. [6] advanced upon modal analysis, developing an IVP (intelligent varying parameter) variant to successfully detect damage in a simulated threestory building.

In order to use modal analysis to accurately locate and quantify damage, a complete "snapshot" of the structure in an undamaged state is required. However, obtaining this snapshot is often difficult to accomplish. It is not always possible to measure all the necessary modal parameters before the structure is damaged. Depending upon how the structure is loaded, certain modal parameters will vary, leading to inaccurate detection. The sensors used to measure the modal parameters are prone to noise disturbances, and depending on sensitivity, would either record too much noise, or not be able to define the damage enough.

Physical structural simulation data may not be feasibly accessible for research purposes. In many cases, advanced simulations are utilized instead for the generation of the needed data. These are often complex finite-element models created to realistically mimic various civil structures such as bridges [10], dams [11], and multi-level buildings [12]. The sensor data used in this thesis is generated using a MATLAB coded finiteelement model provided by the American Society of Civil Engineers (ASCE). The model 
will generate the acceleration response of an undamaged building as well as different structural faults of varying degrees of severity. ASCE's Datagen program will be described in more detail in Chapter 6.

\subsection{Feature Extraction}

Feature extraction involves processing the acquired data in order to make it relevant. Since many SHM systems are based on modal analysis, algorithms which work upon vibration frequencies and amplitudes are often employed. Fourier transforms and

fast-Fourier transforms (FFT) are an example. Morelier et al. [13] focused on using Fourier Descriptors to successfully track damage on a CFCF (cantilever free cantilever free) plate simulation on ABAQUS software.

More recently, wavelet transforms have become a popular tool for feature extraction. An improvement upon Fourier transforms, wavelet transforms can retain temporal information relevant to damage detection. Much research has been done in implementing wavelet transforms in SHM systems. Hou et al. [14] chose a Daubechies DB4 mother wavelet and performed a first level wavelet decomposition upon a multispring/mass model with breakable springs. He successfully demonstrated that the coefficients visually registered the damage (Figure 2.2). 


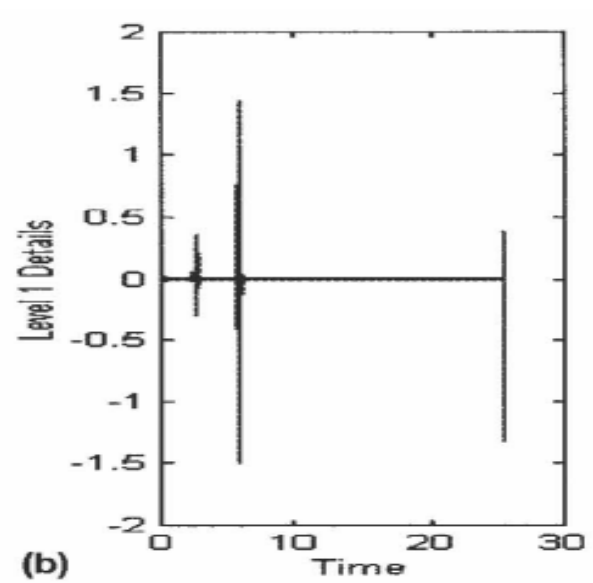

Figure 2.2: Level 1 decomposition coefficients showing spikes at damage [14]

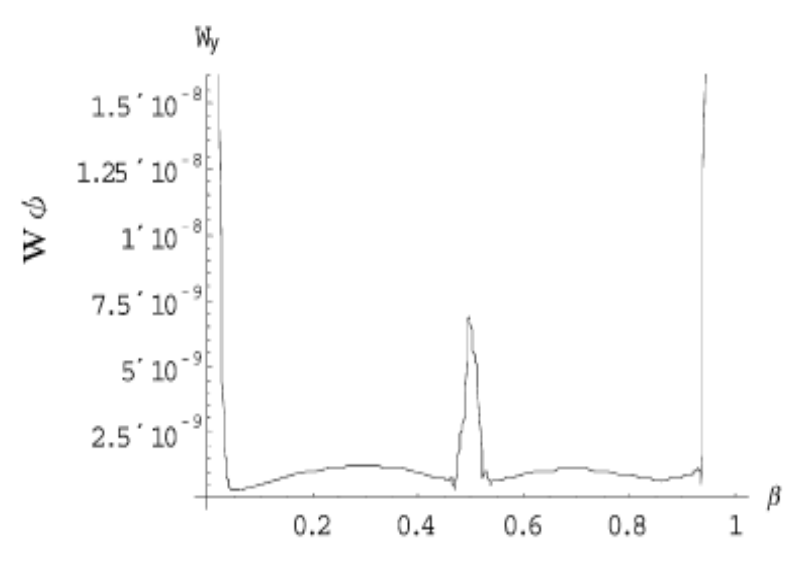

Figure 2.3: Level 3 decomposition coefficients showing spike at damage [15]

Chang et al. [15] used a spatial wavelet approach to detect local perturbations in order to locate the crack location on a Timoshenko beam. He used the Gabor wavelet family and 3rd level coefficients, which showed a spike where the damage occurred (Figure 2.3).

Since most wavelet transform operations are accomplished using computers, the discrete wavelet transform (DWT) is often employed. Lotfollahi-Yaghin et al. [11] simulated an undamaged and damaged arch concrete dam using ABAQUS software. An undamaged and damaged dam model was created using ABAQUS and the $0-100 \mathrm{~Hz}$ response was fed into MATLAB's wavelet toolbox. Various mother wavelets were tested (Coif, Bior, Dmey, Db, etc), with many showing a spike where the dam cracked. It concluded that wavelet detail coefficients can be successfully utilized to detect the damage and also localize the spatial position of the crack.

Huang et al. [10] simulated the response of a five-span arch bridge disrupted by an impulse actuation. The bridge had a length of $440 \mathrm{~m}$; The deck was around $22.5 \mathrm{~m}$ wide 
and $35 \mathrm{~m}$ above the ground. The bridge was supported by rollers in the longitudinal direction, but was constrained in the transverse and vertical directions at both ends, which were separated from the adjacent spans by expansion joints. The transverse impulsive force was generated by suddenly braking a loaded truck that weighed about 15 tons and was traveling in a direction with an inclined angle of $30^{\circ}$ from the centerline of the deck. The resulting acceleration data captured by the sensors is then processed with a DWT. The result showed the 9 mode shapes with frequencies less than $5 \mathrm{~Hz}$ were changed sufficiently to detect the braking response from the truck.

The DWT is also often used for data compression. This is shown in [3] where the DWT is used to process sensor data from a module before the module transmits it to a location. A finite element model of the Humbolt Bay Bridge is used to simulate remote wireless sensors recording acceleration data. Each sensor performs wavelet transforms from levels $1-9$ on the 4,500 features it collects. This allows it to reduce the transmission bandwidth up to $88 \%$.

Wu et al. [16] used level 4 DWT coefficients to detect damage of a machining lathe. The DWT is calculated and the energy is calculated for six approximation and detail levels. The energy values are then re-arranged from the largest to the smallest, and the first few are selected to be used to reconstruct the signal. The cross-correlation between the reconstructed signal and the original signal is calculated. If it is close to one, the lathe is considered undamaged. Wu successfully used the DWT energy method to determine when the lathe began to "chatter" (Figure 2.4), signifying damage. 


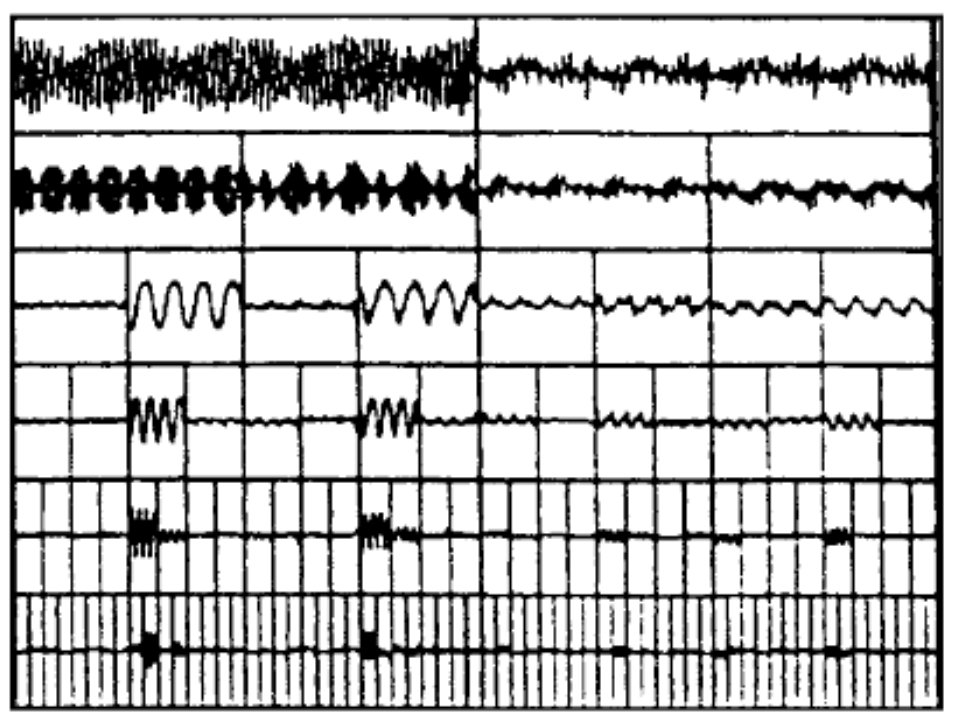

Figure 2.4: Decomposition of a chattering lathe [16]

Choosing a specific wavelet family or "mother wavelet" for a DWT can yield differing success. The selection process has been a research topic itself. Kai et al. [17] proposed in creating a hybrid wavelet neural network (HWNN) where a feed-forward neural network contains DWTs, each using a different mother wavelet as the hidden layer. The test data used was traffic flow of Route 23 in Aichi-ken, Japan on 2003; data was collected every 60 minutes. This is fed into two sub-networks, one consisting of DWTs using the Morlet family, while the other used the Mexican hat wavelet family. two sub-networks are encapsulated by a neural network. The results showed that the HWNN method had a superior MSE of 1.29e-4 compared to using Mexican hat alone (MSE = 19.51) or Morlet alone (4.3e-3). This showed that selecting the right mother wavelet had a significant effect to the results.

Preliminary research has been accomplished in utilizing DWTs for SHM. Hera et al. [18] laid the groundwork by demonstrating that structural damage showed up in wavelet coefficients as “spikes”. ASCE's finite element model Datagen was used to 
generate the necessary undamaged and damaged acceleration data. Five seconds of undamaged data and five seconds of damaged data were processed using the DWT with a db4 mother wavelet. In the first level detail coefficients, a spike is visually present at the time of damage (Figure 2.5). Hera et al. concludes that DWTs can be used to detect possible sudden structural damage, and when it occurred.

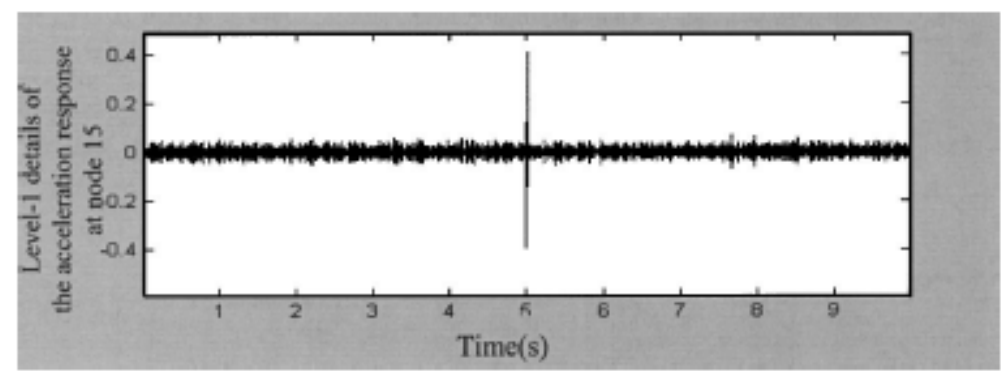

Figure 2.5: Level 1 detail coefficients showing spike at 5 second mark [18]

Mizuno et al. [12] attempted to demonstrate the ability to distinguish structural damage by using "signatures" created by DWT coefficients. These "signature" are a set of quantized wavelet coefficients. Using the same ASCE Datagen model, Mizuno processed 41 seconds of acceleration data using the Haar mother wavelet. The coefficients are then passed through a custom threshold and quantification algorithm. The resulting values represent the damage "signature” of each damage pattern (Figure 2.6). It was able to distinguish between most damage cases. However, the setup could only be operated within single datasets. 


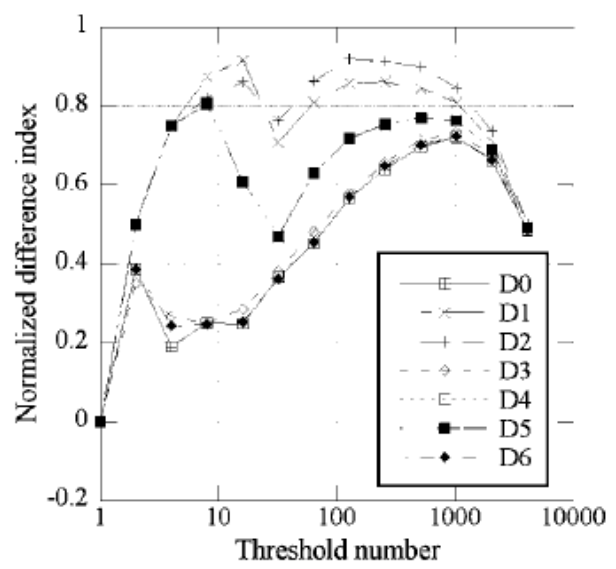

Figure 2.6: "Signatures" of various damage patterns (D0-D6) [12]

In prior research, DWT coefficients are directly used to detect transient structural damage. Damage is often visually confirmed with the existence of a single coefficient spike. In this thesis, DWTs are applied to undamaged, transient, and steady state structural damage of 9 damage patterns. DWT coefficients are used to calculate the energy ratio in percentages, which is then used with an ANN or AIS model to detect the damage state of the structure.

\subsection{Model Development}

The model development portion of SHM involves using the extracted features to create models such that one of the following questions is answered: (1) Is there damage in the structure (existence)? (2) Where is the damage in the structure (location)? (3) How severe is the damage (extent)? [2]. Various techniques and methods can be employed in creating these models. 
Traditionally, this is accomplished by developing mathematical or analytical models. This is not always possible in real life due to the complexity of the structure. Alternatively, experimental structural modeling techniques require only sensor data in order to operate.

Artificial neural networks (ANNs) is a robust method often used for modeling complex and nonlinear systems. If both undamaged and damaged experimental structural data is present, ANNs can be used to model various structural states. Kao et al. [19] showed how accurately neural networks are capable of modeling structure damage. First, neural system identification networks (NSINs) are trained using structural data of varying damage status. Once the NSINs are trained, they are then used to generate free vibration responses. The generated responses are then analyzed and compared to the original response to determine how accurately the NSINs modeled each damage amount. The feasibility of this method is demonstrated using acceleration data recorded from a 3m long, $2 \mathrm{~m}$ wide, $6.5 \mathrm{~m}$ high steel structure mounted on a shake table. The table is shaken at $8 \%, 10 \%, 20 \%, 40 \%$, and $52 \%$ of the strength of the Kobe earthquake. Five NSINs were set up to detect each damage percentage. Each NSIN consists of 301 neurons in the input layer, 0 neurons in the hidden layer, and 5 neurons in the output layer. The 301 inputs correspond to 250 structural accelerometers and 51 external accelerometers. The activation function used is the ramp function. The NSIN is trained for 200,000 cycles using a limited memory Broyden-Fletcher-Goldfarb-Shanno (L-BFGS) back propagation method. The system error between the free vibration responses generated from the outputs compared with the actual response is less than 1.72e-4, showing that each NSIN successfully modeled its damage percentage accurately. 
ANNs have been implemented to detect structural damage. Tsou et al. [20] sought to detect and identify damage in two spring-mass systems (Figure 2.7) using neural networks. Damage is simulated by altering the stiffness of the springs from $100 \%$ down to $10 \%$. For the 3DOF model distinguishing a single damage case, a 3 layer feed forward back-propagating neural network consisting of 40 hidden neurons is constructed for each model. The changes of the eigenvalues between the undamaged system and the damaged system are used as the network's three inputs. The network outputs a maximal pattern error. After training through 80,000 iterations, testing results show the maximal pattern error to within $0.5 \%$, which meets the convergent criterion. The 3DOF model distinguishing between multiple damage levels used 3 hidden layers of 100, 60, and 40 neurons. After 60,000-70,000 iterations, testing results show a classification error of $3 \%$. Likewise data from the 8DOF model is fed into an 8 layer feed forward back-propagating network. Testing the neural network shows that the output was within 3\% of the correct value.

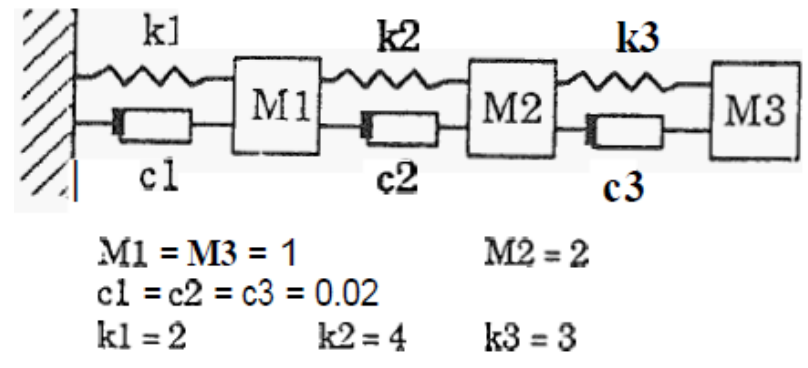

(a)

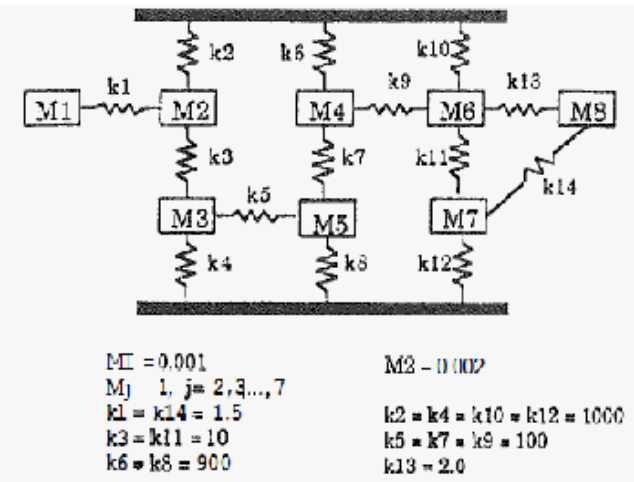

(b)

Figure 2.7: Spring-mass models: (a) 3 DOF model. (b) Kabe’s 8 DOF model [20] 
Sofge [21] used neural networks to predict damage in a cantilevered beam embedded with fiber optic sensors. The first four modal frequencies are recorded for each of 60 different damage configurations. This is fed into a feed-forward neural network with two hidden layers of 9 and 25 neurons. The neural network successfully predicted the new beam dynamics of the damaged beam.

Previously, ANNs have been shown to successfully model relatively simple structures and damage patterns [20]. This thesis will explore the possibility of using an ANN to model a much more complex system- a 120DOF 4 story structure.

More recently, the use of artificial immune system (AIS) algorithms have become popular in the SHM circle. These algorithms each mimic a specific aspect of the biological immune system. Some examples of these can be found in Table 2.1.

Table 2.1: Biological-Artificial immune system equivalent examples [22]

\begin{tabular}{|l|l|}
\hline Biological immune system & AIS equivalent \\
\hline Antibody & Feature vector with class information \\
\hline Antigens & Training and classifying data \\
\hline B-cells & Artificial B-cells \\
\hline Immune memory & Memory cells \\
\hline Primary response & Antigenic stimulation to the antibody set \\
\hline
\end{tabular}

Chen et al. [22] applied multiple AIS algorithms in his Artificial Immune Pattern Recognition (AIPR) approach in order to classify structural damage in two models. The AIPR revolved around training, mutating, and cloning antigens (Figure 2.8). When mature antigens are created, they are stored in the memory cell set, used in the subsequent step to detect structural damage. 


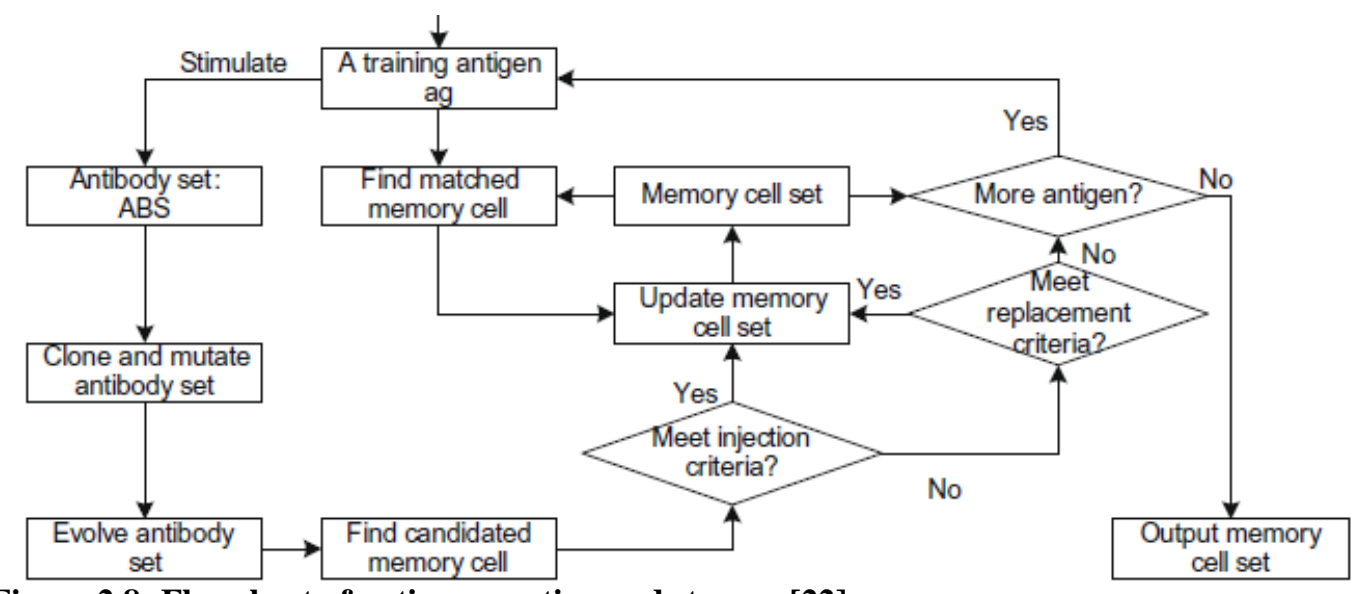

Figure 2.8: Flowchart of antigen creation and storage [22]

Structural data is simulated using ASCE's Datagen model, as well as a three-story frame provided by the Los Alamos National Laboratory. Datagen's four most severe damage patterns were simulated for a duration of 2 seconds. A total of 1,750 datasets were generated for training. The sensor data from the 16 accelerometers for each set is compressed using principal component analysis (PCA). Feature extraction is then accomplished on the data via multiple regression analysis. The results are used to train the AIS algorithms. The AIPR is then tested with 300 datasets. The result showed that it was $80.2 \%$ accurate in classifying damage. A similar setup was performed using the Los Alamos Nation Laboratory's frame. The AIPR resulted in an average success rate of 75.2\% for classifying damage.

This thesis will build upon the AIS research mentioned above. It will focus on the negative selection (NS) algorithm, which is a streamlined and more robust version of the antibody approach. The NS algorithm is explained in more detail in chapter 5. 


\section{CHAPTER 3 : WAVELET TRANSFORM}

The wavelet transform is a useful tool for decomposing a signal into subcomponents for the analysis purposes. These subcomponents, known as wavelets, are similar to the sinusoidal components of Fourier transform decompositions. However, wavelet decomposition retains temporal information whereas Fourier analysis does not. This is possible due to the finite nature of wavelets. Temporal information can be extracted through the processing of the approximation and detail levels. Each decomposition level decimates the temporal resolution by 2 . The general formula for the temporal resolution $\boldsymbol{\tau}$ is:

$$
\tau=\frac{L(x)}{2^{n}}
$$

where $L(x)$ is the length of the input, and $n$ is decomposition level.

For example, a second level wavelet decomposition of a 60 second signal sampled at $1 \mathrm{~Hz}$ will yield coefficients that have a resolution of 4 seconds. This time information allows wavelet transforms to detect transient signal disturbances which would normally be lost through Fourier analysis.

\subsection{Mother Wavelet}

Wavelet analysis requires the selection of an appropriate "mother wavelet". Different mother wavelets affect the effectiveness of the wavelet transform [10]. Figure 3.1 plots some of the popular mother wavelets: (a) Daubechies db6, (b) Mexican hat, (c) Gaussian, (d) Morlet. Research has been done in utilizing multiple "mother wavelets" in 
the algorithm [17], but this thesis will focus upon finding and using a single optimal "mother wavelet".

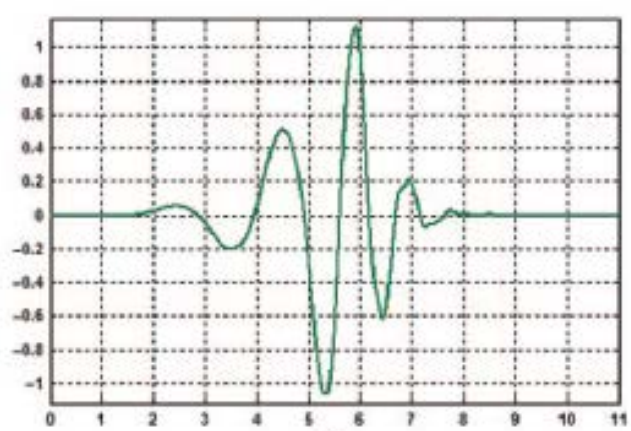

(a)

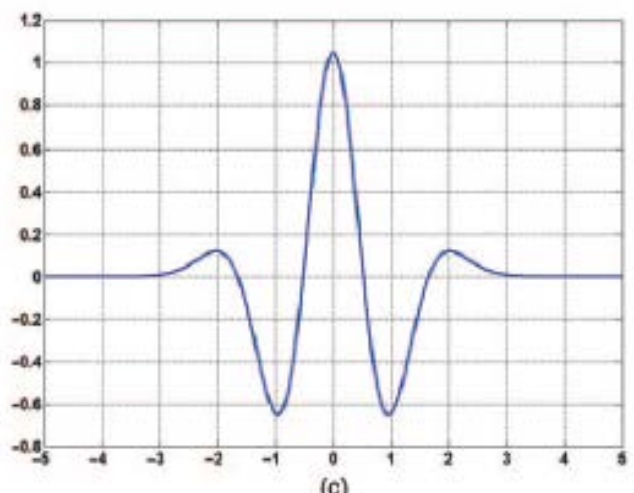

(c)

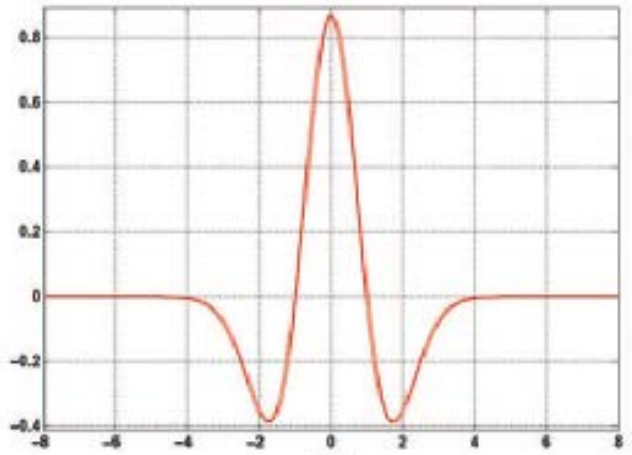

(b)

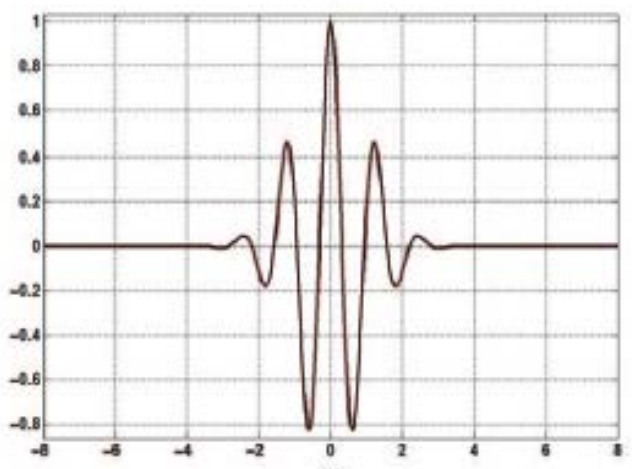

(d)

Figure 3.1: Some mother wavelet examples [23]

A waveform $\Psi(t)$ must meet the following criteria in order to be considered as a wavelet.

The waveform must have finite time duration.

The waveform must contain finite energy

Figure 3.1 shows some of the many mother wavelets. Once the "mother wavelet" $\Psi(t)$ is selected, a family of wavelets is generated. This is accomplished by using scaling factor $s$ and shifting factor $\tau$ on $\Psi(t)$. This creates scaled and shifted child wavelets:

$$
\Psi\left(\frac{t-\tau}{s}\right)
$$


The continuous wavelet transform of a signal $f(t)$ would then be:

$$
T(\tau, s)=\frac{1}{\sqrt{s}} \int_{-\infty}^{\infty} f(t) \Psi^{*}\left(\frac{\mathrm{t}-\tau}{\mathrm{s}}\right) \mathrm{dt}
$$

$\Psi^{*}$ indicates complex conjugate.

\subsection{Discrete Wavelet Transform}

In signal processing applications and in this thesis, the discrete wavelet transform (DWT) is used. This is accomplished by discretizing the scaling factor $s$ and shifting factor $\tau$. This is often accomplished by dyadic discretization such that:

$s=2^{a} \tau=2^{a} b$, where $a$ and $b$ are positive integers.

Replacing those into (3.3) yields discrete child wavelets

$$
\psi_{a, b}(t)=\frac{1}{\sqrt{2^{a}}} \Psi\left(\frac{t-2^{a} b}{2^{a}}\right)
$$

which simplifies to

$$
\psi_{a, b}(t)=2^{-\frac{a}{2}} \Psi\left(2^{-a} t-b\right)
$$

Thus, the DWT of function $f(t)$ would be

$$
\lambda_{a, b}=T(a, b)=2^{-\frac{a}{2}} \int_{-\infty}^{\infty} f(t) \Psi^{*}\left(2^{-a} t-b\right) d t
$$


While the inverse DWT would be

$$
f(t) \sum_{a} \sum_{b} \lambda_{a, b} \psi_{a, b}(t)
$$

In a DWT, a signal is deconstructed into details and approximations. The detail $D$ at level $n$ is defined as

$$
D_{n}=\sum_{b} \lambda_{n, b} \psi_{n, b}(t)
$$

While the approximation $A$ at level $n$ for a transform down to $\mathrm{N}$ is defined as

$$
A_{n}=\sum_{m>n}^{N} D_{m}+A_{N}
$$

Each detail and approximation level contains a set of unique $\lambda_{a, b}$ coefficients. These coefficients contain frequency and temporal information and are very useful for signal processing applications. Figure 3.2 shows how wavelet coefficients are structured.

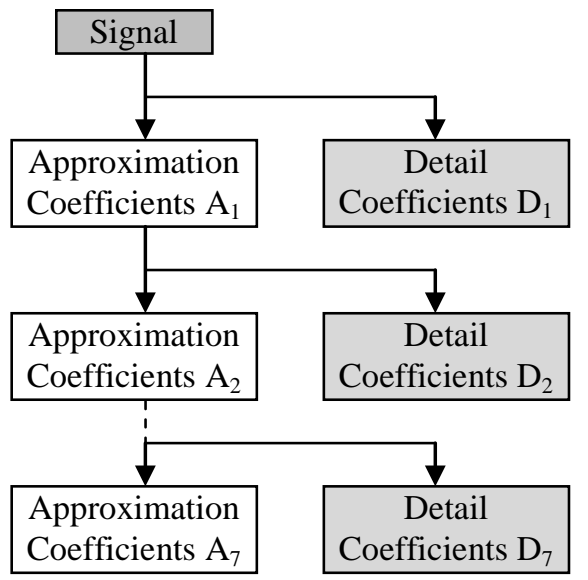

Figure 3.2: Wavelet decomposition coefficient structure overview 


\subsection{Using MATLAB's Wavelet Toolbox}

For this thesis, wavelet transforms are accomplished using MATLAB's built-in wavelet toolbox. The graphical toolbox was used to visualize the effects of the decomposition, while the command line was used to actually process the acceleration response used in the thesis. Figure 3.3 shows an example of a wavelet decomposition. The signal $s$ is the sensor data of a structure excited by wind. The $\mathrm{x}$ axis counts the 40,000 samples the sensor recorded. At sample 20,000, damage is introduced to the structure. The subplots $d_{1}-d_{7}$ show the detail wavelet coefficients of decomposition levels 1-7 respectively. Finally, the subplot $\mathrm{a}_{7}$ shows the coefficients at approximation level 7.
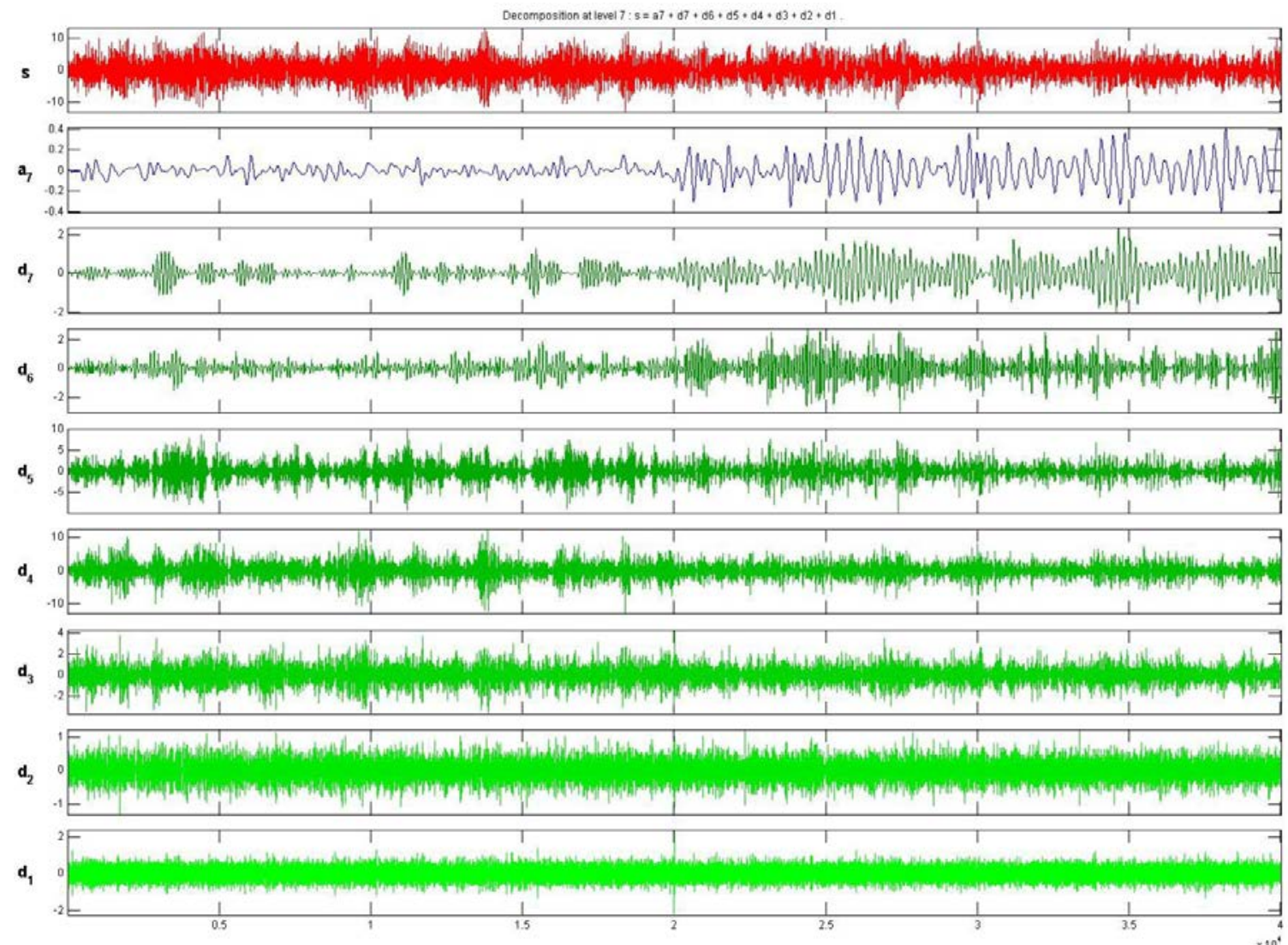

Figure 3.3: Sensor data decomposition example 
The command line used is:

$$
[\mathrm{C}, \mathrm{L}]=\text { wavedec}\left(\mathrm{X}, \mathrm{N}, \mathbf{w}^{\mathrm{w} n a m e}\right)
$$

$\mathrm{C}$ is a vector that contains the actual approximation and detail values.

$\mathrm{L}$ is a bookkeeping vector and contains the lengths of each approximation and detail level in vector C. See Figure 3.4 for a detailed view of the vector structure.

$\mathrm{X}$ is the name of the data array which to perform the wavelet transform on.

$\mathrm{N}$ is the maximum decomposition level the wavelet transform will reach

'wname' is the name of the mother wavelet used to calculate the transforms.

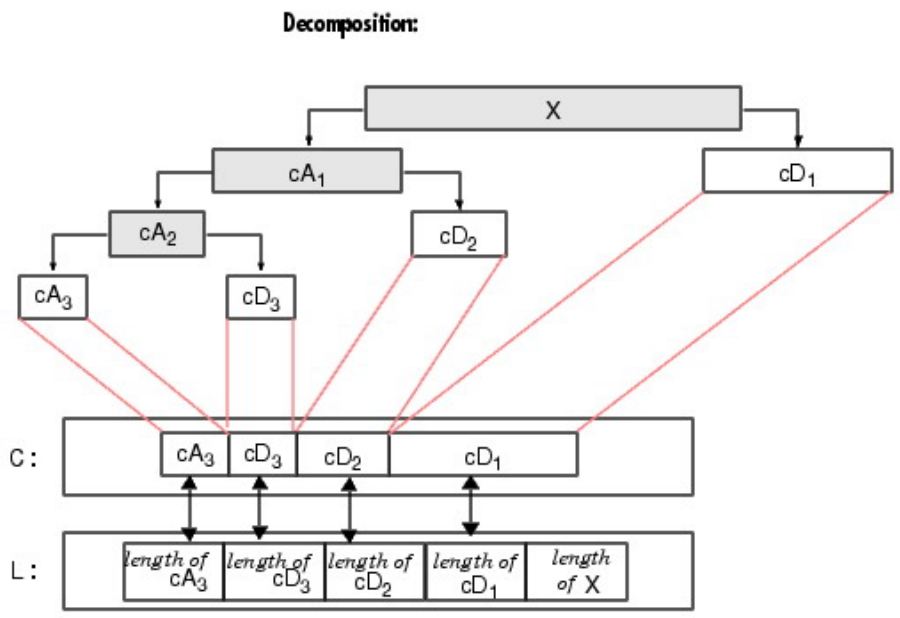

Figure 3.4: L vector overview [24]

Using the settings, wavedec first takes in the data array of length $\mathbf{N}$. It then produces a high-pass and a low-pass filter of length $2 \mathbf{N}$ based upon the selected mother wavelet. It convolves the data array with the filters and then decimates/downsamples the result by 2 . The coefficients generated from the high-pass form the detail level and the 
coefficients generated from the low-pass form the approximation level. This forms one level of decomposition. In order to obtain the next level of coefficients, the approximation level is processed through the wavelet transform again in a similar fashion.

\subsection{Edge Padding}

Transforming a finite length data array using wavelets can lead to artifacts near the beginning and the end of the array. MATLAB's discrete and continuous wavelet transforms deal with this issue by padding the data array in a variety of ways. Table 3.1 lists a few different methods available in MATLAB.

Table 3.1: Edge padding options

\begin{tabular}{|c|l|}
\hline Mode & \multicolumn{1}{c|}{ Description } \\
\hline 'sym' & $\begin{array}{l}\text { Symmetric-padding (half-point): boundary value symmetric replication -- } \\
\text { default mode }\end{array}$ \\
\hline 'symw' & Symmetric-padding (whole-point): boundary value symmetric replication \\
\hline 'asym' & $\begin{array}{l}\text { Antisymmetric-padding (half-point): boundary value antisymmetric } \\
\text { replication }\end{array}$ \\
\hline 'asymw' & $\begin{array}{l}\text { Antisymmetric-padding (whole-point): boundary value antisymmetric } \\
\text { replication }\end{array}$ \\
\hline 'zpd' & Zero-padding \\
\hline 'spd' & Smooth-padding of order 1 (first derivative interpolation at the edges) \\
\hline 'sp0' & Smooth-padding of order 0 (constant extension at the edges) \\
\hline 'ppd' & Periodic-padding (periodic extension at the edges) \\
\hline
\end{tabular}

The default mode is 'sym'. This can be changed by calling the MATLAB command dwtmode('mode') 
Below are some examples of how border padding affects the same set of wavelet coefficients. Figure 3.5 shows the effect of using 'sym' padding. Edge artifacts are clearly visible. Switching the padding option to 'zpd', as shown in Figure 3.6, resolves this issue.

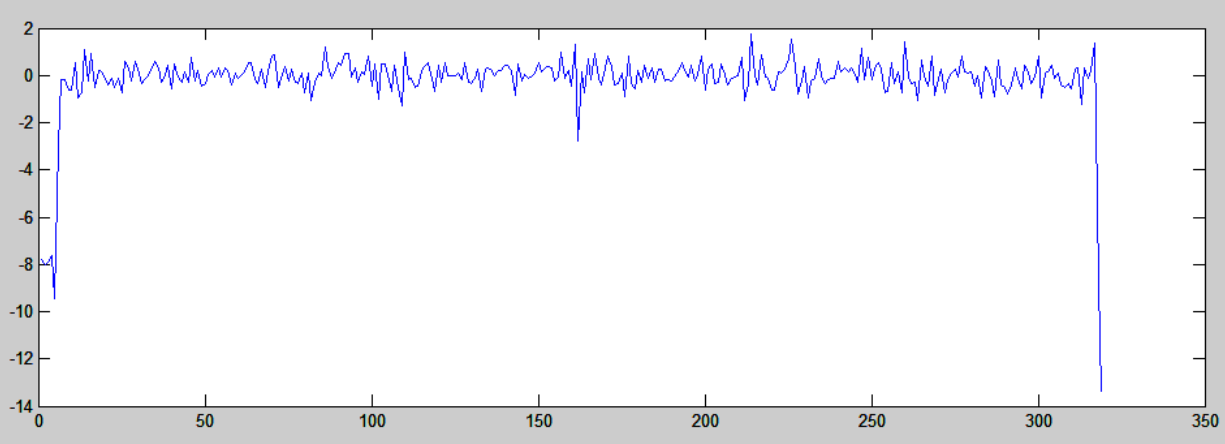

Figure 3.5: cA7 coefficients with default 'sym' padding. Both edges distorted.

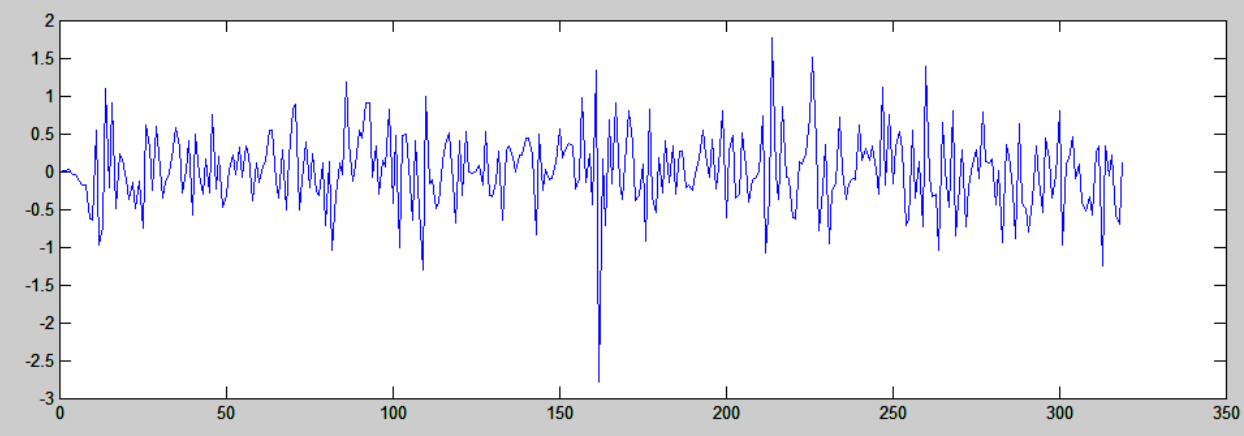

Figure 3.6: cA7 coefficients with 'zpd' padding. No edge distortion

\subsection{Energy Ratio}

The numerical results from a discrete wavelet transform is an array of coefficients, representing either the details or approximations of that level. While it is shown that at certain decomposition levels, a spike formed by a single coefficient often occurs at the moment of damage (see Figure 3.6 for an example), nothing can be formally concluded from that observation. For any analysis of the DWT coefficients to be 
mathematically relevant, the entire array of coefficients must be considered, not just single coefficients. This thesis approaches this by using the array to calculate the energy ratio of the DWT coefficients.

To calculate the energy ratio, the total energy of the sample is first calculated. This is accomplished by performing the DWT of the signal to the first level. The resulting set of $n$ detail coefficients $D_{1}$ and approximation coefficients $A_{1}$ are used to calculate the energy $E_{t}$ :

$$
E_{t}=A_{1}^{2}+D_{1}^{2}
$$

Similarly, let $C$ to be the coefficients of the specific decomposition level in interest. If it contains $m$ coefficients, the specific energy $E_{s}$ is calculated:

$$
E_{s}=\sum_{i=1}^{m}\left(C_{i}\right)^{2}
$$

Finally, the energy ratio $E_{r}$, represented by percentage, can be calculated as

$$
E_{r}=\frac{E_{s}}{E_{t}} \cdot 100
$$




\subsection{Summary}

Wavelet transforms can be an impressive tool used to process and prepare a signal for classification. It is able to filter out noise and focus upon a specific band of frequencies. In order for a wavelet decomposition to work effectively, certain parameters have to be set up properly. A mother wavelet must be chosen, the decomposition level and coefficient type must be selected, and proper edge padding must be implemented. Once the coefficients are calculated, a method of interpreting their existence must be defined. While there are certain guidelines upon making these decisions, optimizing the wavelet decomposition for a specific purpose still requires trial and error. 


\section{CHAPTER 4 : ARTIFICIAL NEURAL NETWORKS}

Artificial neural networks (ANN) are being developed and utilized in SHM systems as a NDE method that is built upon modal analysis [25] [20] [21]. It is a distributed information processing system which mimics the human brain. It is valued for its ability to recognize patterns, classify nonlinear data, and map the input-outputs of a system that would be difficult to model. In the realm of SHM, ANN can theoretically be used to map the sensor inputs of a building to its state of health.

Like the human brain, a neural network consists of many simple processors (neurons) that are interconnected with each other. A neuron, shown in Figure 4.1, consists of weights (synaptic weights), a bias, and an activation function. Inputs $x_{1}, x_{2}$, $\ldots x_{\mathrm{n}}$ are multiplied with their individual, adjustable weights $w_{1}, w_{2}, \ldots w_{\mathrm{n}}$. The results are summed with a bias weight $b$ and form the sum:

$$
s=\sum_{i=1}^{n} x_{i} w_{i}+b
$$

The sum is then input into an activation function $f(s)$. A variety of activation functions are available to use depending on the type and range of data. Table 4.1 below lists some of the commonly used activation functions. 
Table 4.1: Activation function examples

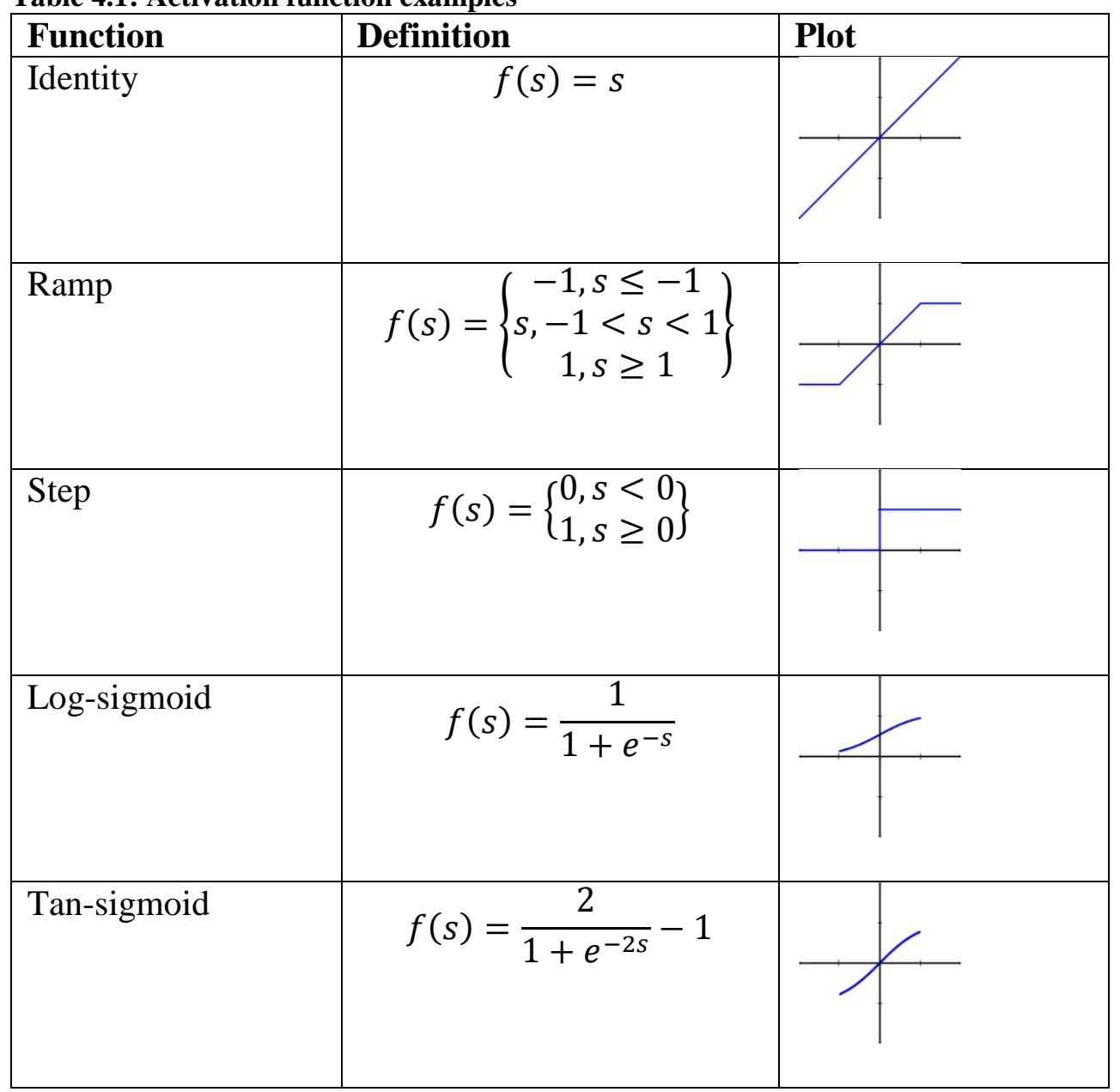

Finally, the neuron outputs $y=f(s)$. Depending on what it is connected to, the neuron output is either an input for another neuron, or the final output of the neural network.

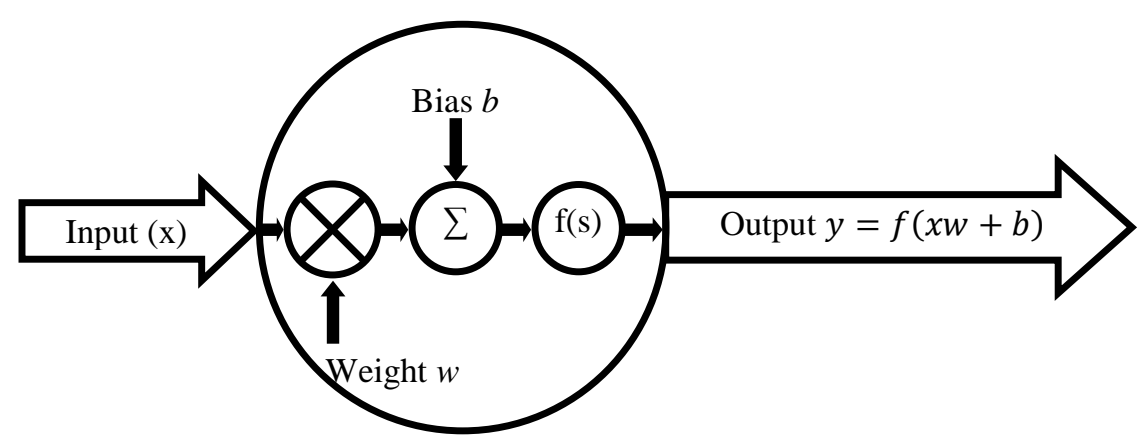

Figure 4.1: Single input neuron model 
There are three layers of neurons that form a neural network: the input, hidden, and output layer. The input layer consists of a number of neurons equal to the data width. These neurons output to the hidden layer, which consist of multiple interconnected neurons in multiple layers. Finally, the hidden layer connects to the output layer, which consists of a number of neurons equal to the desired output data width. Figure 4.2 shows an example of a neural network with an input layer consisting of 4 neurons, two hidden layers with 3 neurons each, and an output layer of one neuron.

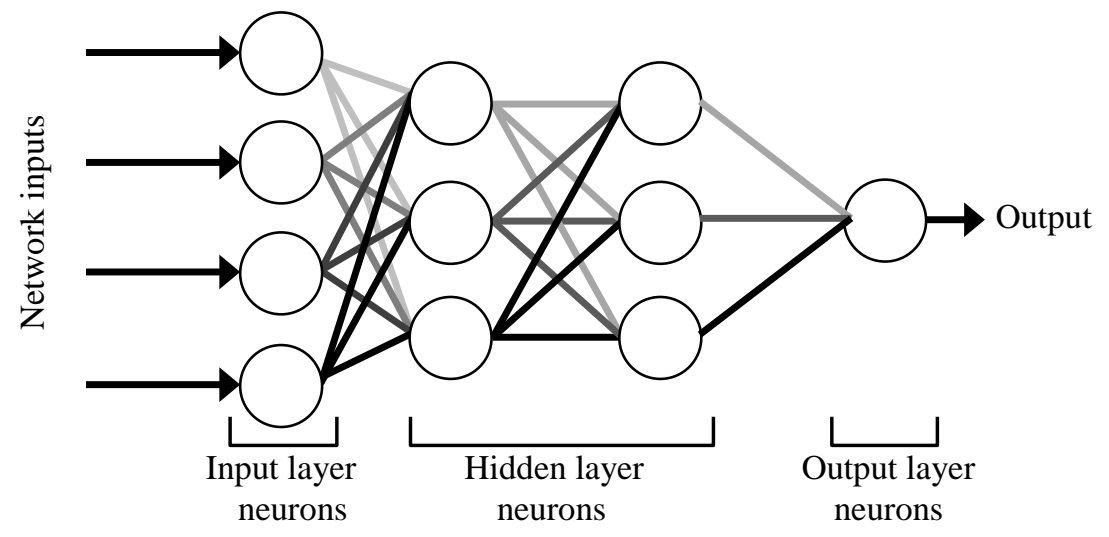

Figure 4.2: Neural network example

\subsection{Training ANNs}

A neural network needs to be trained before it functions properly. The network is set up with a specific number of neurons per layer based upon the input and output data width. Each neural link is seeded with an initial weight. Each neuron is seeded with an initial bias. A set of training input-output data is then used to "teach" the network. There are multiple learning methods available; the one focused on this thesis is feed-forward back propagation. In this method, input training data is fed through the input and passed through the entire network. The network output is then compared to the training output. 
The difference between the network output and training output is used to adjust weights. The adjustment is "back propagated", meaning the weights of the connections closest to the outputs are adjusted first.

In back propagation, the amount each weight is adjusted is dependent on what kind of neuron it is part of. For the output layer neurons, the weight change is defined as $\Delta w=\eta \varepsilon f^{\prime}(s) y$ where $\mathrm{y}$ is the output and $\varepsilon$ is the difference between the desired training output and network output. $\eta$ is the learning rate, which controls how fast the network conforms to the training data. Small values of $\eta$ lead to slower learning, but large values may cause to network oscillate and never reach steady state. For the hidden layer neurons, the weight change is defined as $w_{\text {new }}=w_{\text {old }}+\eta \varepsilon f^{\prime}(s) y$. The connection weight adjustment continues backwards until the input layer neurons are adjusted, as shown in Figure 4.3.

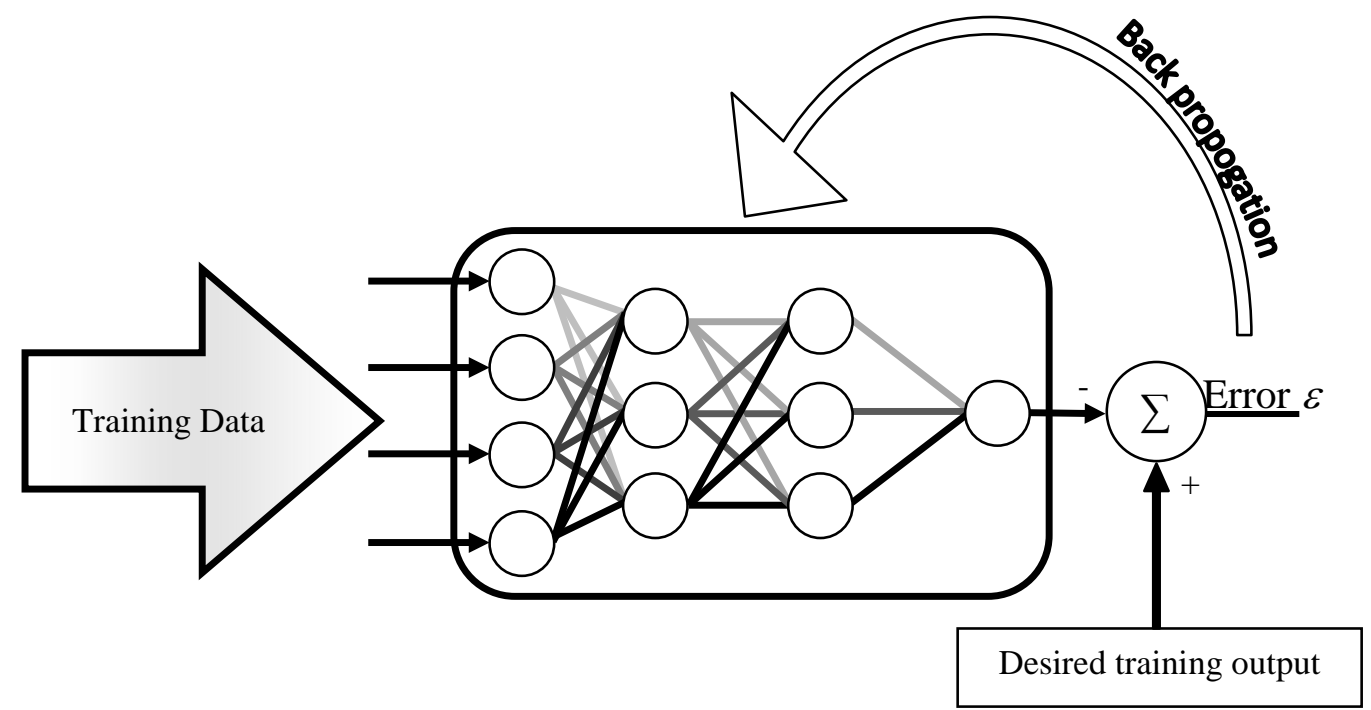

Figure 4.3: Back propagation 
Through this experiential training, the connection strength (synaptic weight) between each neuron adjusts such that the input-output matches the training data within an acceptable level of error. The Mean Square Error (MSE) is often used to gauge this. It is calculated as

$$
M S E=\frac{1}{2 N} \sum_{i=1}^{N} \varepsilon_{i}^{2}
$$

where $N$ is the total number of training iterations, and $\varepsilon_{i}$ is the difference between the training output and the network output per instance. Once the MSE is acceptably low, the training ends and neural network is ready to be used.

\subsection{Using MATLAB's ANN Toolbox}

This thesis uses MATLAB's built in neural network toolbox to evaluate the effectiveness of artificial neural networks as a NDE method. The command is this:

$$
\text { net=newff(P,T,Si,Tfi); }
$$

creates a new feed-forward back-propagating neural network named 'net'. 'P' is the training input; ' $\mathrm{T}$ ' is the training output; ' $\mathrm{Si}$ ' is a vector detailing the number of neurons in the first layer, and each subsequent hidden layer; 'Tfi' is a vector detailing the transfer function of each neuron layer. For a feed-forward back propagating neural network, certain parameters affect its performance. These are detailed below. 


\subsection{Number of Neurons and Hidden Layers}

The quantity of neurons per layer and the number of hidden layers affects the complexity and adaptability of the neural network. Neural networks with more neurons and hidden layers can potentially model more complex problems accurately. However, increasing the number of neurons and hidden layers will not always yield better results. Because of increased nonlinear modeling capability granted by more neurons, the network can be more sensitive to noise. In addition, each additional layer adds more processing power requirements. In the end, choosing the right number of neurons and hidden layers requires systematic trial and error regime in order to create an effective neural network.

\subsection{Transfer Function}

The transfer functions used by the hidden layers and the output layer in a neural network can affect the performance of the network. Certain transfer functions work well with certain outputs. For example, the 'logsig' works comparatively well with Boolean outputs. Choosing the right transfer function for each layer requires systematic trial and error.

\subsection{Training Algorithm}

The training function controls how the neural network output error is backpropagated to update the weight and bias of each neuron. These are often based on the gradient descent. The training function used in this thesis is a built-in MATLAB function 
based on the Levenberg-Marquardt optimization. It is a quasi-Newton method which approximates the Hessian matrix as:

$$
H=J^{T} J
$$

$\mathrm{J}$ is the jacobian matrix that contains the first derivatives of the network errors with respect to the weights and biases. The Levenberg-Marquardt algorithm uses this approximation to calculate the update:

$$
x_{k+1}=x_{k}-\left[J^{T} J+\mu I\right]^{-1} J^{T} e
$$

$I$ is the identity matrix, $\mu$ is the variable training rate, and $e$ is a vector of network errors. The LM algorithm is the most efficient MATLAB method for training neural networks up to a few hundred weights [26] and is thus selected for this thesis.

\subsection{Summary}

Artificial neural networks are a good candidate for NDE methods based upon its ability to model complex nonlinear systems. In real life, a structure is often too complex to be accurately portrayed using finite-element modeling. As long as the neural network is given sufficient training data, it can dynamically shape itself into the necessary model.

The major shortcoming for an ANN is that it must be trained with the entire spectrum of data before it can be used. In terms of SHM, it means that in order to detect structural damage, the ANN must be trained with damaged and undamaged data. In 
addition, ANNs work much more effectively when trained with both noise-free and noisy data. Unfortunately both training requirements may not be feasible or available depending upon the structure. 


\section{CHAPTER 5 : ARTIFICIAL IMMUNE SYSTEM}

A biological immune system is a complex system capable of detecting and neutralizing foreign substances. It accomplishes this through the means of complex pattern recognition across a vast distributed network. As a result, the system is highly capable of dealing with previously un-encountered pathogens and boasts of strong heuristic ability [27].

In the 1990s, scientists developed a new branch of computational intelligence based upon the biological immune system. Referred as artificial immune system (AIS) algorithms, these mimic various aspects of the biological immune system. Within this set, an algorithm known as negative selection (NS) is developed. NS is based upon the adaptive branch of the immune system and imitates the behavior of B-cells and T-cells [28].

In brief, the biological NS operates like this: countless T-cells are generated, each created with a pseudo-random collection of surface receptor sites. These are filtered within the thymus, which hosts cells that have "self" receptor sites. If the T-cell's receptor site binds with any of the host cells within the thymus, it is killed off. Otherwise, the T-cell is allowed to exit the thymus and circulate in the body. If anything binds with the T-cell in the body, it is considered a "non-self" and the T-cell triggers it to die. Figure 5.1 gives an overview of this process. 


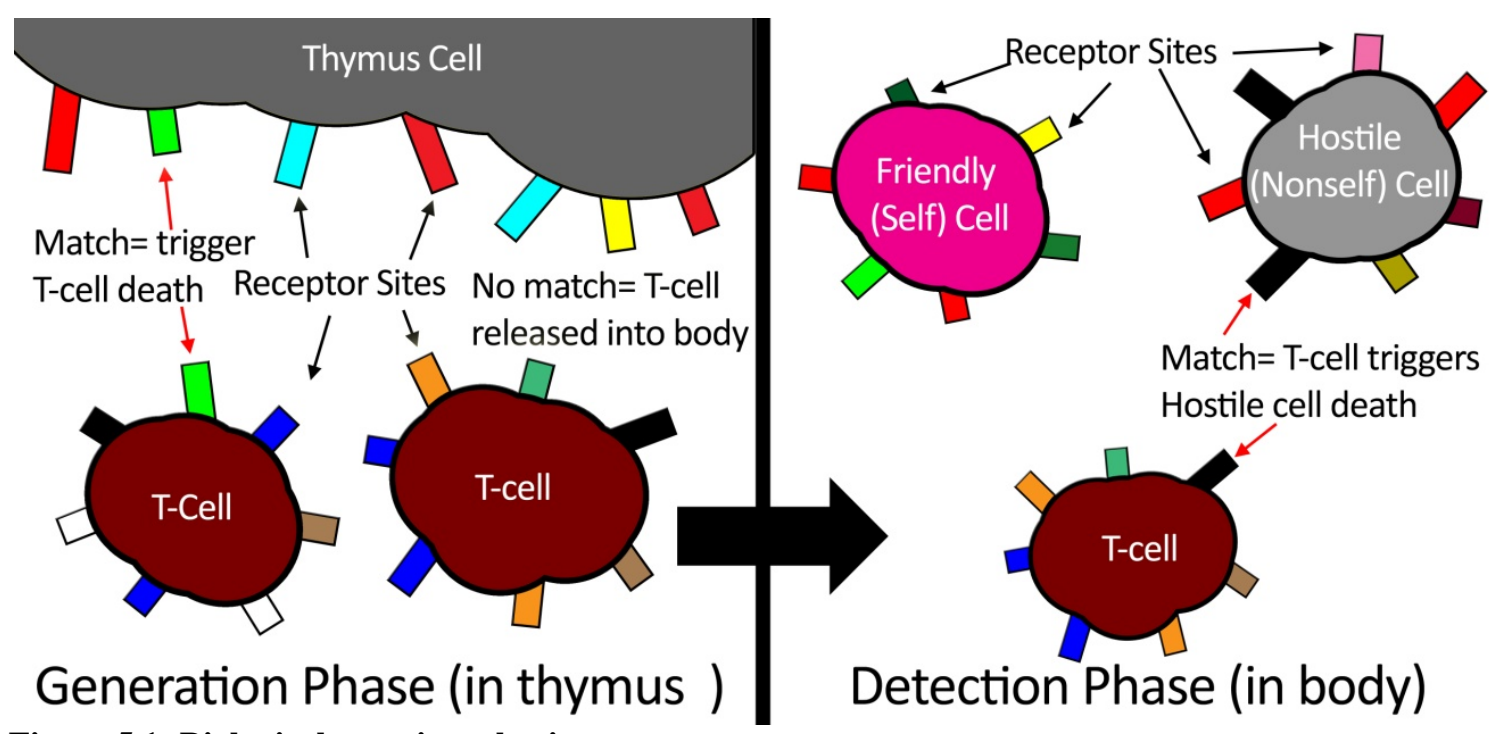

Figure 5.1: Biological negative selection

An artificial negative selection algorithm works in a similar fashion and is accomplished in two phases: A generation phase and a detection phase, shown in Figure 5.2. During the generation phase, the algorithm produces a detector with a number of randomly generated initial coefficients equal to the data width. Training data constituting "self” values are fed to a comparator. If any of the detector's receptor sites are deemed similar to the training data or to any previously surviving detector, it is eliminated. Otherwise, the detector is retained. The algorithm continues creating random detectors until a certain criteria is met, whether it is the number of detectors, or it fails to generate a valid detector over a certain number of attempts. When that is the case, the NS algorithm is "trained" and the detection phase begins. In this phase, each unknown data set is compared with the entire set of trained detectors. If the unknown data is deemed similar to any detector, it is considered "non-self”. Otherwise, it is deemed self. 


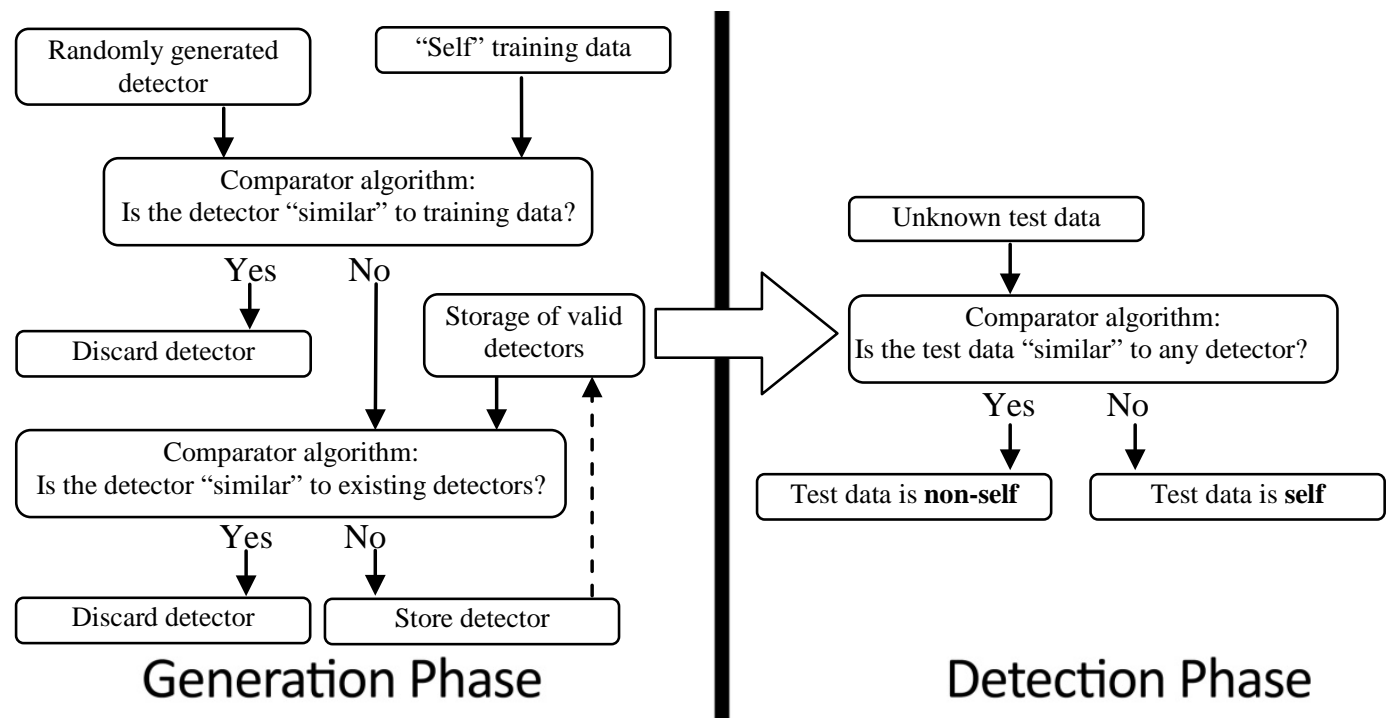

Figure 5.2: Artificial negative selection algorithm

A simple demonstration of the NS algorithm is shown in Figure 5.3. In Figure 5.3, the NS algorithm is given two dimensional training data denoted by red dots. Using this training data, the algorithm generates detectors denoted by the blue circles to cover the “non-self” space. This is known as the generation phase. Once this is complete, the detection phase begins and the NS algorithm is given testing data. This is shown as dots in Figure 5.3(b). Using the array of detectors created during the generation phase, the NS algorithm now determines if each testing data point is considered "self”, or "non-self”. Anything that falls within a detector range is considered "non-self"; this is denoted by coloring the dot red in Figure 5.3(b). If the data does not fall within any detector range, it is considered to be "self” and is denoted by coloring the dot green. 

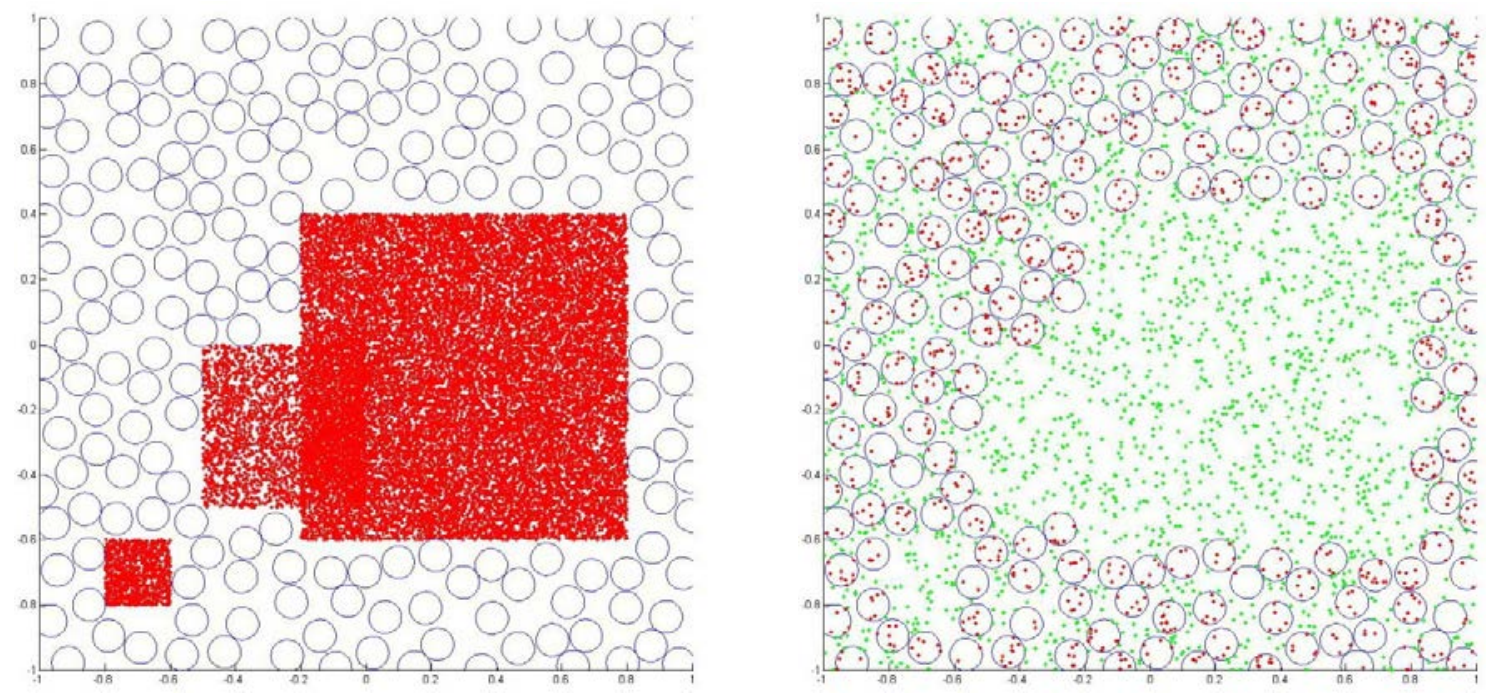

Figure 5.3: (a) NS algorithm fixed radius training and (b) testing

Two aspects of the negative selection algorithm must be considered in order to create an effective detection system: similarity criteria, and coverage.

\subsection{Similarity Criteria}

A detector is eliminated when its receptors are deemed "similar enough" to the training data, and an unknown data set is considered "non-self" when it is "similar enough" to a detector. That is because in reality, complete exactness highly improbable. Therefore, a metric called "similarity criteria" must be defined. "Similarity criteria" is how similar two things may be in order to be considered the same. Mathematically speaking, there are many options in determining "similarity criteria". The most commonly used metric a n-norm distance threshold. Given two data points, $P_{1}=$ $\left(x_{1}, x_{2}, \ldots x_{n}\right) P_{2}=\left(y_{1}, y_{2}, \ldots y_{n}\right)$. In one dimension, if the distance between the two 
points is within a threshold $\left|x_{1}-y_{1}\right| \leq d$, the points are considered "self". This can be extrapolated into 2 dimension data:

$$
\sqrt{\left(x_{1}-y_{1}\right)^{2}+\left(x_{2}-y_{2}\right)^{2}} \leq d
$$

Or 3 dimensions:

$$
\sqrt[3]{\left|\left(x_{1}-y_{1}\right)^{3}\right|+\left|\left(x_{2}-y_{2}\right)^{3}\right|+\left|\left(x_{3}-y_{3}\right)^{3}\right|} \leq d
$$

Up to n dimensions

$$
\left[\sum_{i=1}^{n}\left|\left(x_{i}-y_{i}\right)^{n}\right|\right]^{\frac{1}{n}} \leq d
$$

The "similarity criteria” chosen has effect on a detector's efficiency, controlling what the detector's coverage shape is. The threshold value $d$ determines the performance of the detector. If $d$ is too small, the detector will be too sensitive to be useful. Otherwise, if $d$ is too big, the detector will be too sloppy and present false positives.

\subsection{Coverage}

A negative selection algorithm is only as effective as the coverage of its detectors. In the biological immune system model, T-cells are given multiple receptor sites to 
broaden its ability to detect "non-self". In a NS algorithm, this is represented where detectors are built with a detection range.

\subsubsection{Fixed Radius}

In a fixed radius setup, a NS algorithm's generation phase create random detectors with a predetermined range. An n-norm method is often used, such that in 2 dimensions, the detector would have a circular detection range, and in 3 dimensions, the detector would have a spherical detection range. The generated detector then checks if any "self" training data is within its detector range, and if any other detectors are within range. If so, it is considered too similar to self and eliminated. The generation phase continues until sufficient coverage is achieved. Figure 5.3 demonstrates an example of a fixed radius NS algorithm operating with 2 dimensional data. The red dots forming the blocks in Figure 5.3 represent the training data inputted into the NS algorithm. The blue circles represent the detectors generated along with their detection radius. These detectors are then used to classify unknown data in Figure 5.3(b), represented by all the dots. Red dots in Figure 5.3(b) represent unknown data that is classified as "non-self", while green dots in Figure 5.3(b) represent unknown data that is classified as "self".

\subsubsection{Variable Radius}

The use of a fixed radius setup often leaves inadequate "non-self" coverage. This is seen in Figure 5.3(b), where many testing data points have erroneously been labeled as "self”. Research has been done in optimizing a detector's coverage. One issue with fixed- 
radius detectors is that there will be undesirable gaps. Variable radius detectors can be implemented to alleviate that issue. During the generation phase, a detector first checks if it's within the radius of a pre-existing detector. If not, it then calculates the shortest distance to another available object using n-norm (5.3), whether it is a training point or another detector radius. It then sets that distance as its detection radius. Figure 5.4 below demonstrates this in a two-dimension example.

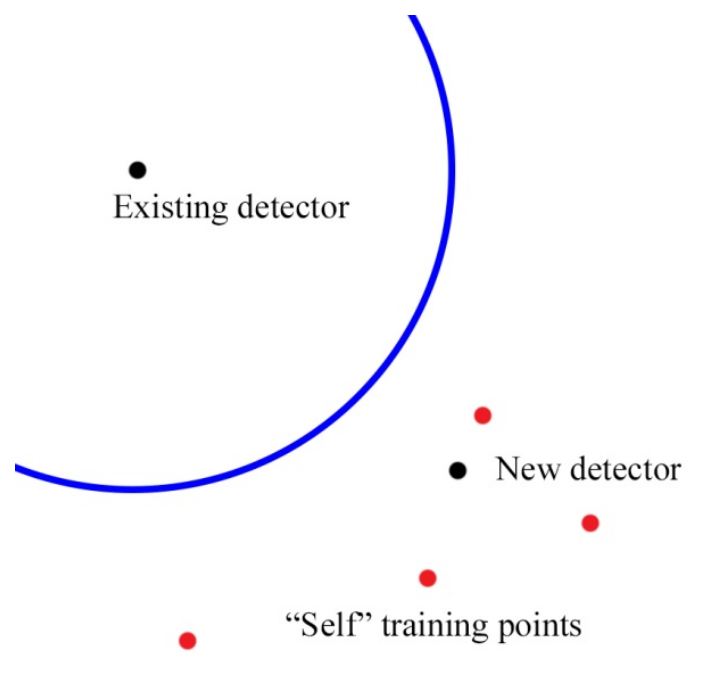

Calculate n-norm distance to nearest object

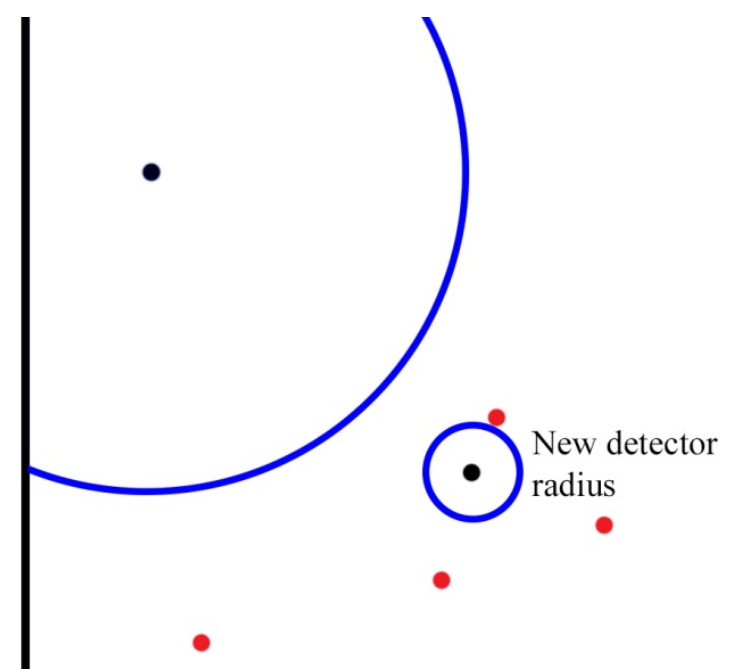

Generate detector radius equal to minimum

Figure 5.4: Variable radius detector generation example

Figure 5.5 shows a variable radius implementation upon the same training data used in Figure 5.3. Figure 5.5 shows the NS generation phase. The red dots forming the blocks are training inputs; the blue circles represent detectors with their respective radii. Figure 5.5(b) shows the resulting detection phase using the same detectors. The red dots in Figure 5.5(b) represent unknown data being classified as "non-self", while the green dots represent the unknown data being classified as "self". 
Using a variable radius allows each detector to be much more efficient in covering the "non-self" space. More gaps can be filled with smaller radii detectors, as shown in Figure 5.5(b). This allows the NS algorithm to return less false "self" classifications.
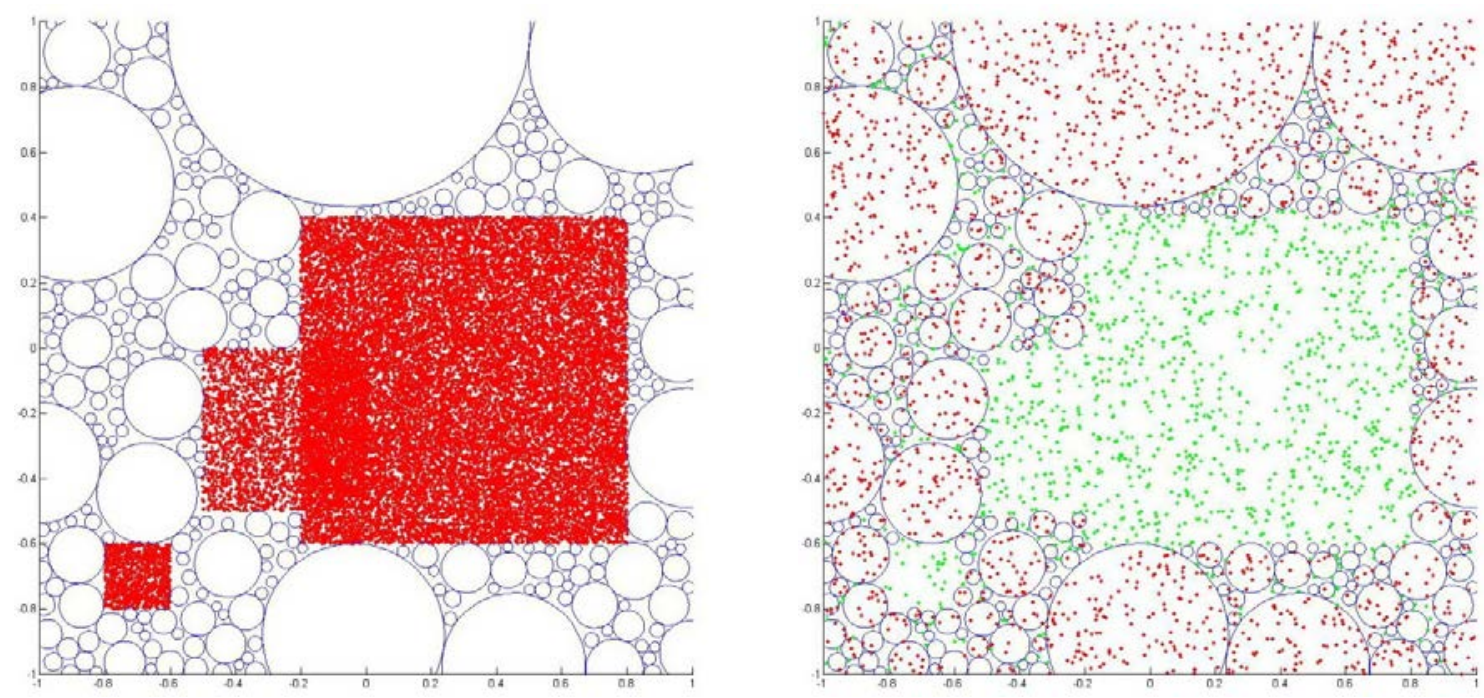

Figure 5.5: (a) NS variable radii training and (b) testing

\subsubsection{Overlapping Radii}

Another technique to improve the NS algorithm's coverage is to implement overlapping detector radii. This is accomplished by allowing the generation of detectors which encroach upon each other's detection range slightly. This is set by a percentage value which states how much each individual detector's radii can be overlapped. Using this method gains the benefit of covering even more gaps which would otherwise return false "self" classifications. 
Combining this technique with the variable radii detectors yields the best results. Figure 5.6 shows a NS algorithm implementing both variable radii and coverage overlap. The algorithm is trained with the same data as the fixed radius example (Figure 5.3), and variable radii only example (Figure 5.5). These are then used in the testing phase shown in Figure 5.5(b). Once again, the red dots in Figure 5.5(b) represent unknown data that the detectors have classified as "non-self", while the green dots represent unknown data the detectors classified as "self". The example shows impressive results; the NS algorithm returns much less false "self" classifications than the previous implementations.

This thesis implements both the variable detection radii as well as overlapping detection coverage techniques in all simulations and results. See Appendix B for MATLAB sample code.
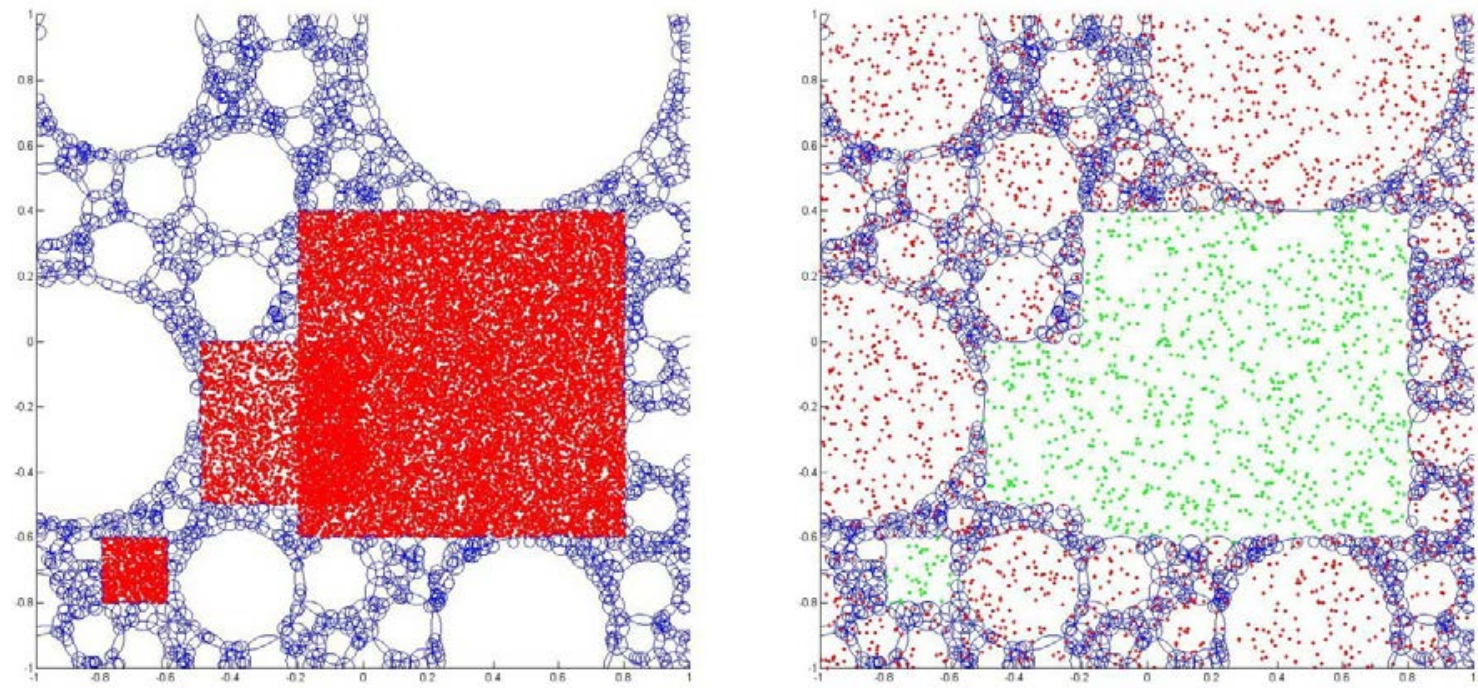

Figure 5.6: (a) Variable radii, overlapping detectors training and (b) testing 


\subsection{Summary}

Negative selection can be easily used as a NDE method. A structure in its current state can have various structural parameters recorded to train a NS algorithm. That would be considered the "self" state. Once the NS algorithm is trained sufficiently with "self" data, it would be able to detect if the structure enters a "non-self" state. Since buildings tend to deteriorate rather than improve, a "non-self" state would mean that something has become structurally wrong.

The benefit of NS over modal analysis is that NS does not require a mathematical model of the structure. It only requires measured experimental data to create the "self" snapshot. As it generates its own "non-self” space, NS is more robust than ANN systems, which requires training with both "self" and "non-self" data.

Data width affects the effective coverage capability of the negative selection algorithm. The "non-self" space which detectors must cover expands exponentially depending on how complex the data is [29]. Various techniques, such as those described above, can be employed to mitigate the resulting increase. 


\section{CHAPTER 6 : DATAGEN}

Structural Health Monitoring is a broad field where many studies can be done. However, these studies on new SHM methods often employ an assortment of sensors on different structures, making comparison of the SHM methods difficult. The ASCE Structural Health Monitoring task group created Datagen as a means of unifying the differing test variables and creating a benchmark for SHM methods [18]. A structure is designed; two models are developed from the structure. Various damage cases are available for selection, as well as variables such as noise and sensor parameters. Datagen then outputs raw acceleration data, which can be processed by various SHM methods.

\subsection{Structure}

Datagen's two models are based upon a test structure located in the Earthquake Engineering Research Laboratory at the University of British Columbia (UBC), shown in Figure 6.1. The four story structure is built with a steel frame. The model is $3.6 \mathrm{~m}$ tall and 2.5m wide. Each floor has four bays arranged 2x2, capable of accepting concrete loading slabs.

Two finite element models based on this structure were developed to generate the simulated data. The first is a $12 \mathrm{DOF}$ model that constrains all motion except in-plane translations. In this model, each floor and its beams are considered rigidly attached. Each floor is allowed to translate in the $\mathrm{x}$ and $\mathrm{y}$ direction, and rotate about the center column. This gives it 3 degrees of freedom per floor. The second is a 120DOF model that only requires the floor nodes to have the same horizontal translation and in-plane rotation. The 
columns and floor beams are modeled as Euler-Bernoulli beams in both finite element models. The braces are bars with no bending stiffness [30].

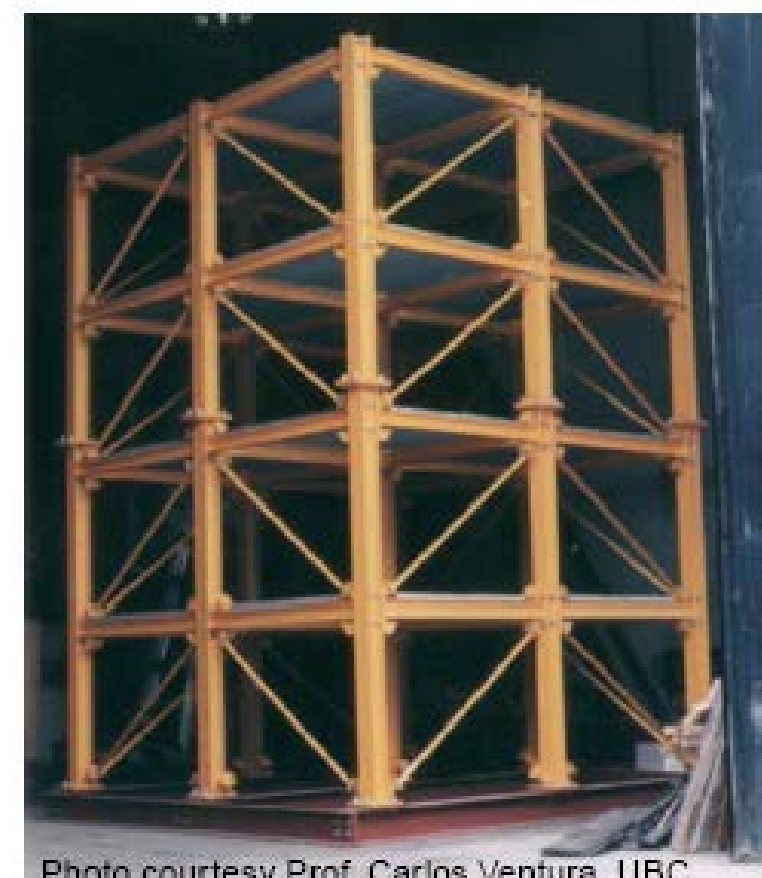

Photo courtesy Prof. Carlos Ventura, UBC

Figure 6.1: Datagen's physical structure model

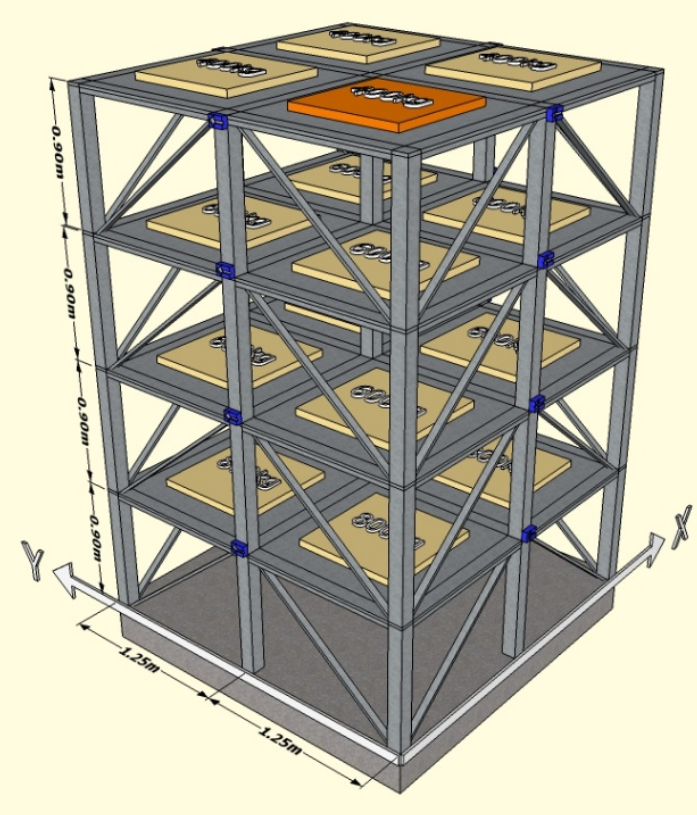

Figure 6.2: Structure model overview

\subsection{Simulation Cases}

Datagen offers five simulation cases, each with a different model and structural load:

Case 1: 12 DOF model with a symmetrical load at all stories, wind excitation Case 2: 120 DOF model with a symmetrical load at all stories, wind excitation Case 3: 12 DOF model with a symmetrical load on the roof, shaker excitation Case 4: 12 DOF model with an asymmetrical load on the roof, shaker excitation Case 5: 120 DOF model with an asymmetrical load on the roof, shaker excitation

\subsubsection{Load Distribution}

Each floor of the model contains 4 bays arranged in $2 \times 2$. When the structure is modeled with load at all stories, it is loaded with $800 \mathrm{~kg}$ slabs on the 1 st floor, $600 \mathrm{~kg}$ slabs on the 2nd and 3rd floors, and 400kg slabs on the 4th floor. 


\subsubsection{Load Symmetry}

A symmetrical load means that each floor's 2x2 bay is loaded with the same weight, while an asymmetrical load means that one of the $400 \mathrm{~kg}$ loads in the top floor's bays is replaced with a $550 \mathrm{~kg}$ slab instead. The bay which has the differing load is marked in orange in Figure 6.2. The default test setup uses a symmetrical load.

\subsubsection{External Excitation}

The external excitation simulated in cases one and two occurs in the form of wind loading. In these simulation cases, an external force is applied in the positive Y direction at each level of the structure. This is shown as blue arrows in Figure 6.3. In cases three, four, and five, the external excitation simulated occurs in the form of a shaker located at the center of the top floor. This shaker excites the structure in both the $\mathrm{X}$ and $\mathrm{Y}$ direction, shown in red arrows in Figure 6.3.

The wind and shaker excitation forces are modeled using Gaussian white noise processes, passed through a 6th order Butterworth filter with a cutoff frequency of 100Hz. The force intensity can be set during simulation. (default 150)

\subsection{Sensors}

The acceleration data generated by Datagen is simulated using 16 sensors. There are four sensors per floor, each located at the center edge column, shown in Figure 6.2. The sensors are aligned such that two are pointed in the $\mathrm{x}$ direction and two are pointed in 
the y direction, as shown in Figure 6.4. Each sensor outputs the magnitude, and the sampling frequency and sensing duration can be adjusted in Datagen.

Each sensor is affected by noise equal to a percentage of the RMS of the combined external excitation force. This percentage can be adjusted during simulation up to $100 \%$. The default is $10 \%$.

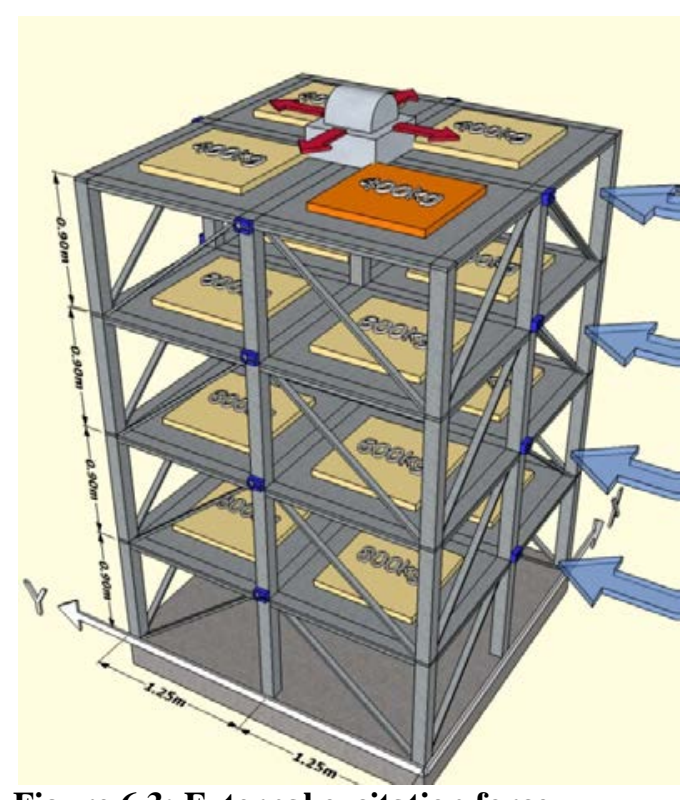

Figure 6.3: External excitation force

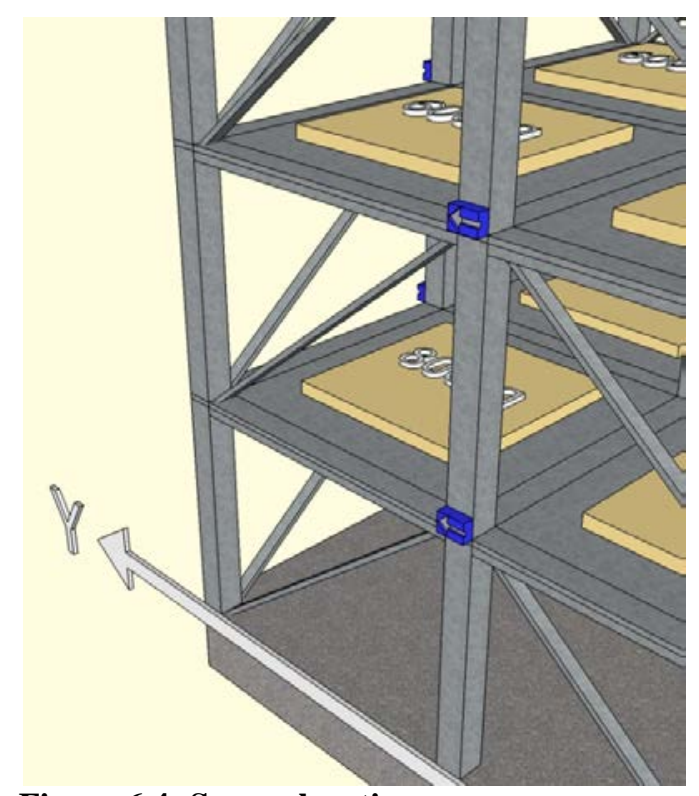

Figure 6.4: Sensor location

\subsection{Damage Simuation}

Datagen simulates damage to the structure by modifying the stiffness value of various elements in the finite element model. An undamaged component has a stiffness value of “ 1 ", while a completely damaged component has a stiffness value of " 0 ”. Datagen comes with six built in damage patterns along with an undamaged pattern. In addition to the built in damage patterns, this thesis includes two addition cases (8 and 9), which are different from the built in ones. Figure 6.1 summarizes the damage patterns visually. 
Table 6.1: Damage pattern summary

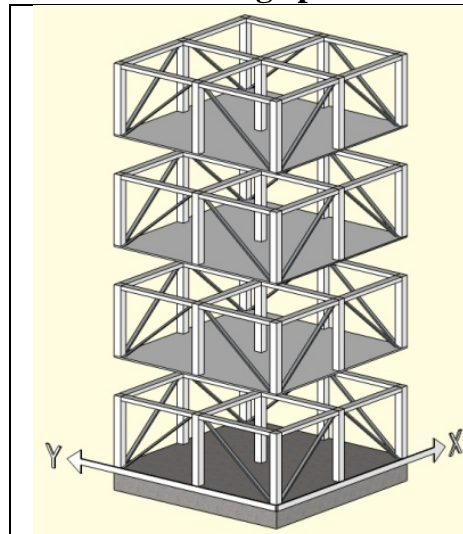

(1) Undamaged

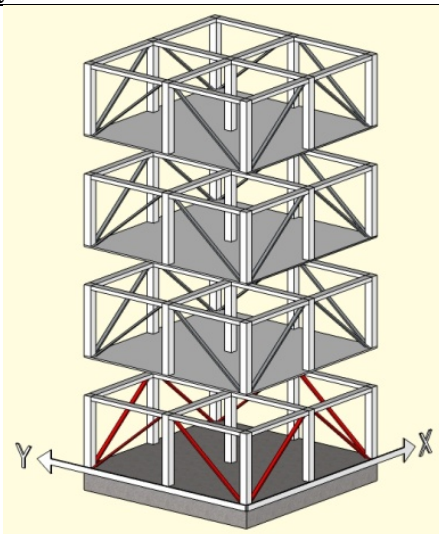

(2) All braces for the 1st floor are broken

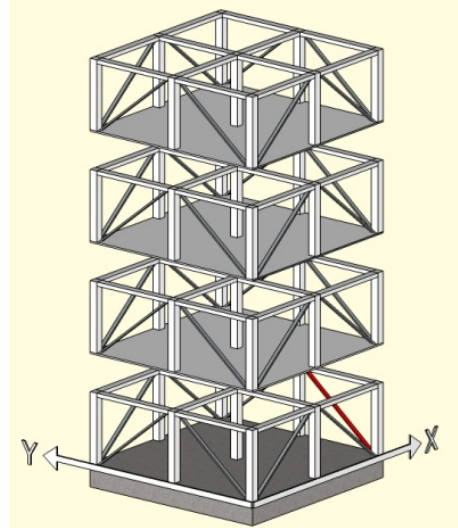

(4) One brace on one side of the 1st floor is broken

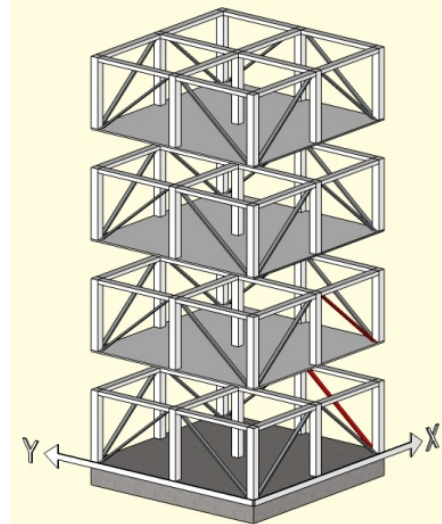

(5) One brace on the 1st and 3rd floor are broken

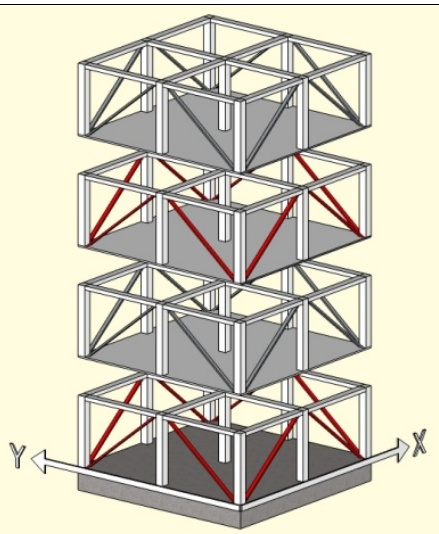

(3) All braces for the 1st and 3rd floors are broken

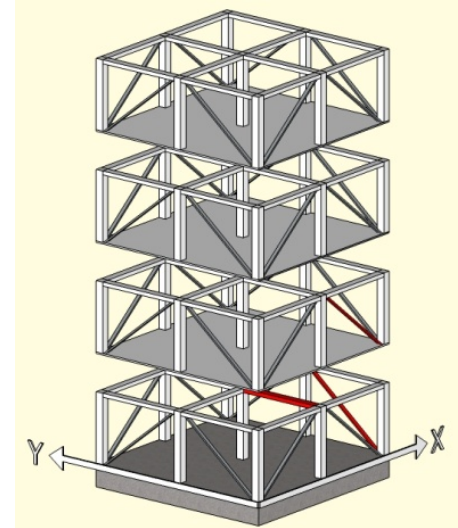

(6) Damage pattern 5 plus the left end of element 18 is unscrewed

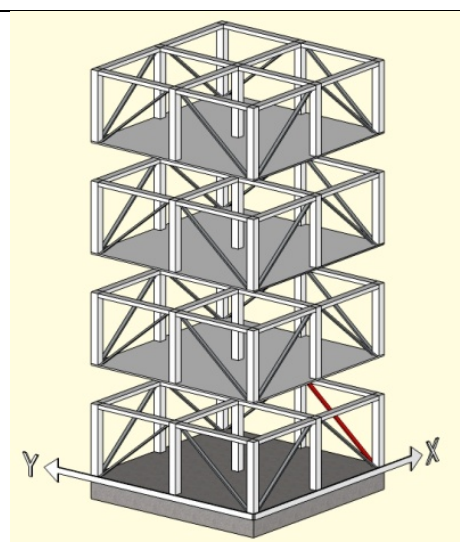

(7) 1 brace at 1st story (element 24), 1/3 cut in area

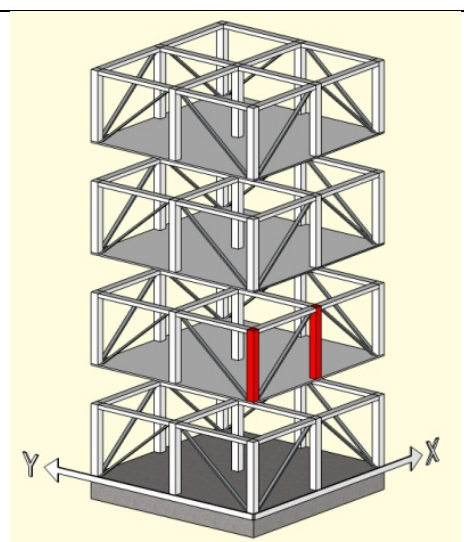

(8) Two columns on the 2nd floor are broken

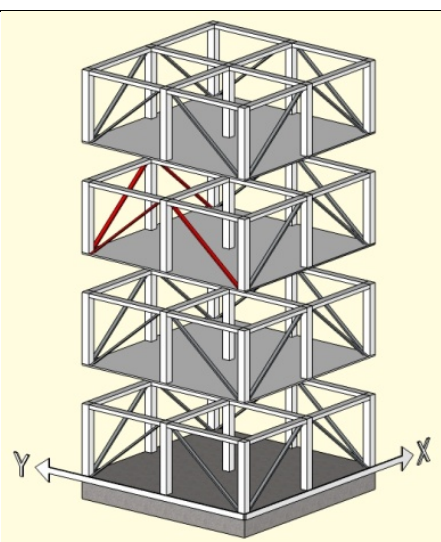

(9) Braces on two sides of the 3rd floor are broken 


\subsection{Running Datagen}

Datagen can be executed using a command line, or a Graphics User Interface (GUI) as shown in Figure 6.5. Either method allows the user to input crucial parameters, which are described below. The command line execution of Datagen allows it to be executed in scripts, as is the case in this thesis.

\begin{tabular}{|c|c|c|c|}
\hline D MENU & 回 $\mathbb{E}$ & \begin{tabular}{|l|l|l|l|}
$\mathrm{DMENU}$ & $\square$ & $\Xi$ & $\mathrm{X}$ \\
\end{tabular} & \begin{tabular}{|l|l|l|l|}
$A$ & ASCE Be... \\
\end{tabular} \\
\hline \multicolumn{2}{|c|}{ ASCE Benchmark Problem: DATA Generation Program } & \multirow[t]{2}{*}{ ASCE Benchmark Problem: DATA Generation Program } & Damping ratio: \\
\hline \multirow{2}{*}{\multicolumn{2}{|c|}{ CASE 1: 12-DOF (symmetric), load at all stories }} & & 0.01 \\
\hline & & Undamaged case & Time step size: \\
\hline \multicolumn{2}{|c|}{ CASE 2: 120-DOF (symmetric), load at all stories } & Damage pattern 1: all braces of 1-st story are broken & 0.001 \\
\hline \multirow{2}{*}{\multicolumn{2}{|c|}{ CASE 3: 12-DOF (symmetric), load at roof }} & & Time duration: \\
\hline & & Damage pattern 2 : all braces of 1 -st and 3-rd story are broken & 40 \\
\hline \multicolumn{2}{|c|}{ CASE 4: 12-DOF (unsymmetric), load at roof } & Damage pattern 3: 1 brace on 1 side of 1 -st story is broken & Noise level: \\
\hline & & & 10 \\
\hline \multicolumn{2}{|c|}{ CASE 5: 120-DOF (unsymmetric), load at roof } & Damage pattern 4: 1 brace on 1 side of 1 -st and 3-rd story are broken & Force intensity: \\
\hline \multicolumn{2}{|l|}{ QUIT } & Damage pattern 5 pattern $4+$ unscrew the left end of element 18 & 150 \\
\hline & & 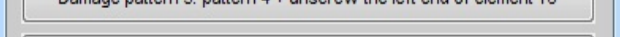 & \multirow{2}{*}{$\begin{array}{l}\text { Seed number for force generation: } \\
123\end{array}$} \\
\hline & & damage pattern 6: 1 brace at 1 -st story (element 24), $1 / 3$ cut in area & \\
\hline \multicolumn{2}{|l|}{ MENU } & & \\
\hline \multicolumn{2}{|c|}{ Use what method in response calculation? } & Damage pattern 7 : columns $30-31$ are broken & \multirow{3}{*}{$\begin{array}{l}1 \\
\text { OUTput DATA file name: } \\
\text { DATAfile } \\
\end{array}$} \\
\hline & & Damage pattern 8: braces $80-83$ are broken & \\
\hline \multicolumn{2}{|c|}{ Isim (You MUST have Control Toolbox) } & & \\
\hline \multicolumn{2}{|c|}{ Nigam-Jennings Algorithm (VERY SLOW) } & User define damage case & OK Cancel \\
\hline \multicolumn{2}{|c|}{ FAST Nigam-Jennings Algorithm (You MUST have SimuLink) } & GuII & \\
\hline QUIT & & & \\
\hline
\end{tabular}

Figure 6.5: Overview of Datagen's GUI menus

This is the command line to run Datagen:

datagen(caseid,damageid,methodid,e,dt,Duration,noiselevel,S,SeedNum,out filename,Findx) 
The input variables of the command line are described below:

\section{Caseid}

There are five simulation cases offered in Datagen as describe above. Each case is a unique combination of structural model, load distribution, load symmetry, and external excitation. The numerical value of this variable corresponds to the case number in this manner: caseid = damage pattern -1 .

\section{Damageid}

Six damage patterns are available for simulation, with the option to add additional user defined patterns. Using this ability, two additional damage patterns are inputted. The damage patterns are described above. The value of this variable corresponds to the damage pattern, e.g. 1 = damage pattern 1 , etc.

\section{Methodid}

Datagen offers three ways to calculate the acceleration response. The first uses MATLAB's Isim command, which uses a discrete-time integration algorithm that assumes excitation is constant over a time step. The other integration algorithm uses the NigamJennings integration that decomposes the system into modal space, integrates each mode assuming the excitation is piecewise linear over a time step, and superimposes to get the time response [30]. The Fast Nigam-Jennings Algorithm is similar to the Nigam-Jennings algorithm, but processes through MATLAB Simulink instead.

All three methods are tested using the same simulation parameters and random seed. The acceleration results from each calculation method varies within $0.02 \%$ with each other. This is a negligible difference when considering the data scope. 


\section{Damping ratio $\mathrm{E}$}

This is the damping ratio used in calculating the building response to external excitation. The default value is 0.01 .

\section{Time interval Dt}

This controls the time intervals in seconds at which the acceleration response is calculated. It can be equated to the acceleration sensors' sampling frequency. The default value is 0.001 .

\section{Simulation Duration}

This variable controls how many seconds of acceleration response Datagen is called to generate. The default value is 40 seconds.

\section{Noiselevel}

This controls the sensor noise level in percent of total RMS of the external excitation forces. The default value is 10 .

\section{External force S}

This variable controls the force intensity of the external excitation, whether it simulates wind or shaker. The default value is 150 .

\section{SeedNum}

This is the numerical seed used when Datagen generates the excitation force,

described in Chapter 3. Each numerical seed will generate unique yet repeatable excitation force response, resulting in a unique acceleration response. The default value is 123. 


\section{Outfilename}

This is a string input which defines what the output datafile will be named. The default value is 'DATAfile'.

\section{FindX}

This is a toggle option which controls whether to use a filter in MATLAB's Signal Processing Toolbox to greatly speed up the response calculation. The default value is 1 (enabled).

\subsection{Output file}

After Datagen completes the requested simulation, it creates a .mat datafile which contains the acceleration response matrix, as well as various record keeping variables. Some of the relevant ones are described in Table 6.2.

Table 6.2: Summary of Datafile variables

\begin{tabular}{|l|l|}
\hline Variable name & Description \\
\hline Duration & Simulated duration, in seconds \\
\hline $\mathrm{K}$ & Transformed system stiffness matrix \\
\hline $\mathrm{M}$ & Transformed system mass matrix \\
\hline $\mathrm{S}$ & External excitation strength \\
\hline Seednum & Numerical seed used to generate noise \\
\hline $\mathrm{T}$ & Transformation matrix used for the rigid-floor effect \\
\hline Acc & Raw acceleration data \\
\hline Caseid & Simulation case number \\
\hline Damageid & Damage pattern number \\
\hline Noiselevel & Sensor noise level \\
\hline
\end{tabular}

From the datafile Datagen creates, the Acc matrix is the primary array used in this thesis. It contains all of the sensor samples for the set duration of the simulation, polled at the desired sampling rate. Figure 6.6 shows a visualized example of all the sensors for 
damage pattern 2, for the duration of 40 seconds. The external excitation force is wind alone, acting on the $\mathrm{Y}$ axis. All even numbered sensors are mounted parallel to the $\mathrm{X}$ axis, while all odd numbered sensors are mounted parallel to the $\mathrm{Y}$ axis (see Figure 6.4). Since the only source of excitation is parallel to the $\mathrm{Y}$ axis, there is almost no response from the odd numbered sensors.

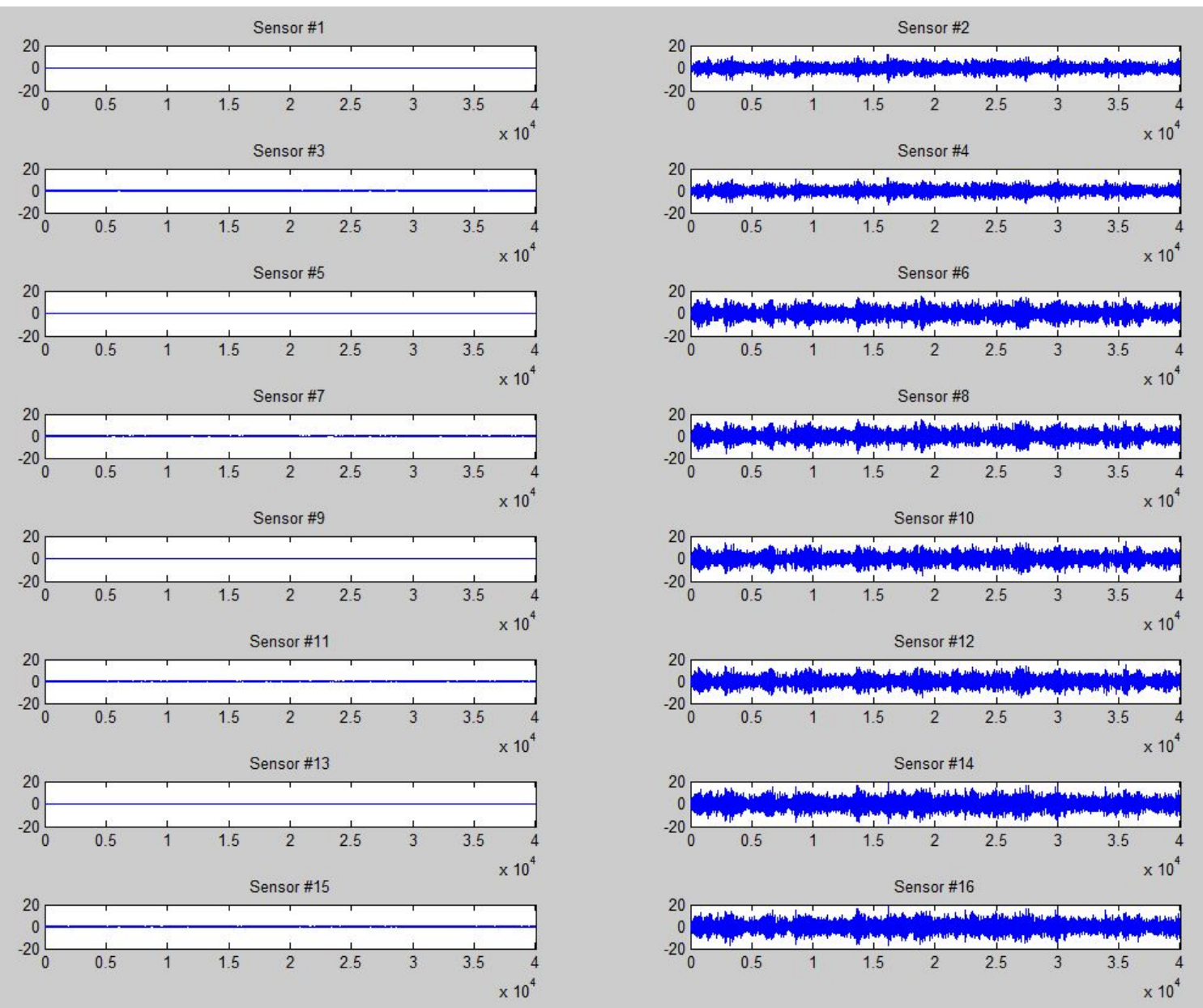

Figure 6.6: Visualized acceleration data for damage case 2 


\subsection{Summary}

Datagen is a robust program which allows the simulation of many types of structural damage scenarios. It implement various levels of simulation complexity, allows different load configurations, and offers different sources of external excitation. In addition, noise at various levels can be introduced in order to mimic reality more accurately. It provides a common platform upon which new and developing SHM technologies can be implemented into and compared with each other. Used as a toolbox, Datagen allows researchers to access and work with a large assortment of simulated data, which would otherwise be difficult to obtain and to replicate. 


\section{CHAPTER 7 : SIMULATIONS}

The process workflow for this thesis can be divided into three phases ,shown in Figure 7.1: data generation, feature extraction, and classification. This chapter will focus on each component, describing variations that are tested, and presenting the most optimal results. See Appendix B for MATLAB code written to encompass the workflow.

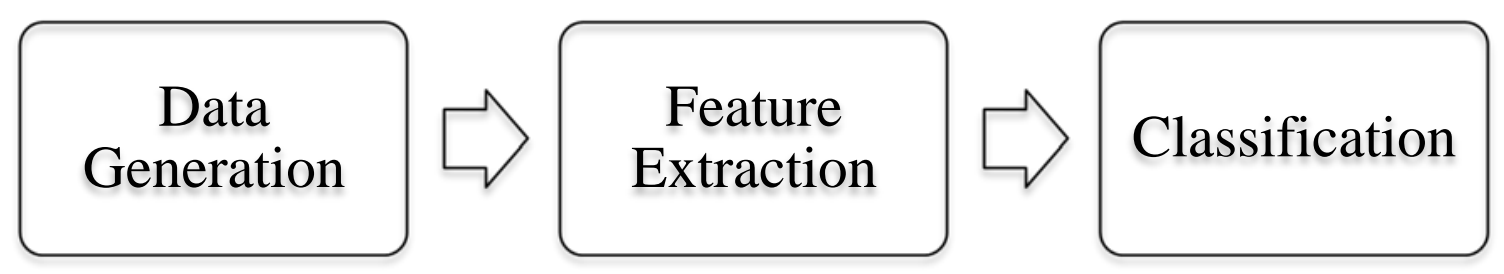

Figure 7.1: Process workflow overview

\subsection{Data Generation}

All of the data used in this thesis is generated using Datagen. Datagen is a versatile software which can be configured to output data in many configurations. The configuration parameters are described in detail in Chapter 3. Since the goal of the thesis focuses upon detail enhancement and model development, many of the Datagen parameters are kept at their default values. The end goal of the data generation phase is to synthesize data matrices containing multiple datasets representing steady state undamaged, steady state damaged, and transient state responses.

For the purpose of this thesis, all results are based upon simulation case 2- 120 DOF model with a symmetrical load at all stories, with wind excitation. All eight damage patterns are simulated and processed. The Isim method for calculating the response is used, as it was the fastest. Since the damping ratio defines the inherent properties of the structure, which is not of interest in this thesis, the default of 0.01 is used. The default 
sampling rate of $1 \mathrm{kHz}$ be used for this thesis. The duration of all data used in the thesis is 1.5 seconds. Sensor noise is left out for the duration. The process is repeated 200 times in order to obtain 200 datasets. The excitation force intensity remains unchanged at the default of 150. Table 7.1 summarizes the generation setup parameters. Please see Appendix B for relevant code.

Table 7.1: Data generation setup parameters in summary

\begin{tabular}{|l|l|}
\hline Parameter & Value \\
\hline Caseid & 2 \\
\hline Damageid & {$\left[\begin{array}{l}0 \\
8\end{array}\right]$} \\
\hline Methodid & 1 \\
\hline E & 0.01 \\
\hline Dt & 0.001 \\
\hline Duration & 1.5 \\
\hline Noiselevel & 00 \\
\hline S & 150 \\
\hline SeedNum & Random([ $\left.\left[\begin{array}{ll}0 & 100\end{array}\right]\right)$ \\
\hline Datasets & 200 \\
\hline
\end{tabular}

\subsubsection{Data Combination}

Datagen itself produces a DATAfile, which is a MATLAB container (described in Chapter 3) that holds record keeping variables as well as the acceleration response. The acceleration response is named acc and is a matrix of $1,500 \times 16$ doubles. The $1^{\text {st }}$ dimension of the acc matrix relates to a time sample and the $2^{\text {nd }}$ dimension relates to the sensor number. Thus, the acc matrix contains 1,501 acceleration samples for each of the 16 sensors.

A MATLAB workflow script has been written to automate the data generation. The script runs through all damage cases and creates a damage matrix by running Datagen multiple times and extracting the acc matrix each time. It then creates a new 
matrix named acc_unm which has dimensions 9x16x1,500 doubles. This matrix of 1,500 acceleration samples for each of the 16 sensors and each of the 9 damage patterns is considered to be a single dataset. 200 of these datasets are generated, with the resulting matrix being 9x16x200x1,500.

\subsubsection{Damage Simulation}

In order to test if various NDE algorithms used in this thesis can detect transient damage, structural data of an undamaged building becoming damaged is required. To simulate this, the stiffness value of various elements are modified from 1 to 0 , depending on the damage pattern. In each dataset's 1.5 second duration, damage is introduced at the half mark (0.75 second). Thus, each sensor effectively outputs undamaged acceleration response for the $1^{\text {st }}$ half of the simulation, and damaged acceleration response for the $2^{\text {nd }}$ half. The method of simulating damage adheres to what is introduced by [18]. This matrix of undamaged-damaged data is called acc_mer. The dimensions are rearranged for ease of use such that $a c c \_m e r$ has the dimensions 9x16x200x1,500 doubles.

\subsubsection{Sensor Reduction}

Since the external excitation force of the model is solely by wind force parallel to the $\mathrm{Y}$ axis, acceleration data measured by sensors mounted parallel to the $\mathrm{X}$ axis can discarded without much loss of detail. In addition, since the current simulations are setup with no sensor noise, the acceleration data from each pair of sensors on the same floor return the same. Consequently, only data from sensor \#4, 8, 12, and 16 are retained for 
further processing. This cuts out $75 \%$ of the data, which are superfluous, and simplifies it down to one sensor for each floor. Figure 7.2 demonstrates this on the first floor of the building. This has the effect of changing the acc_mer matrix to a 9x4x200x1,500.

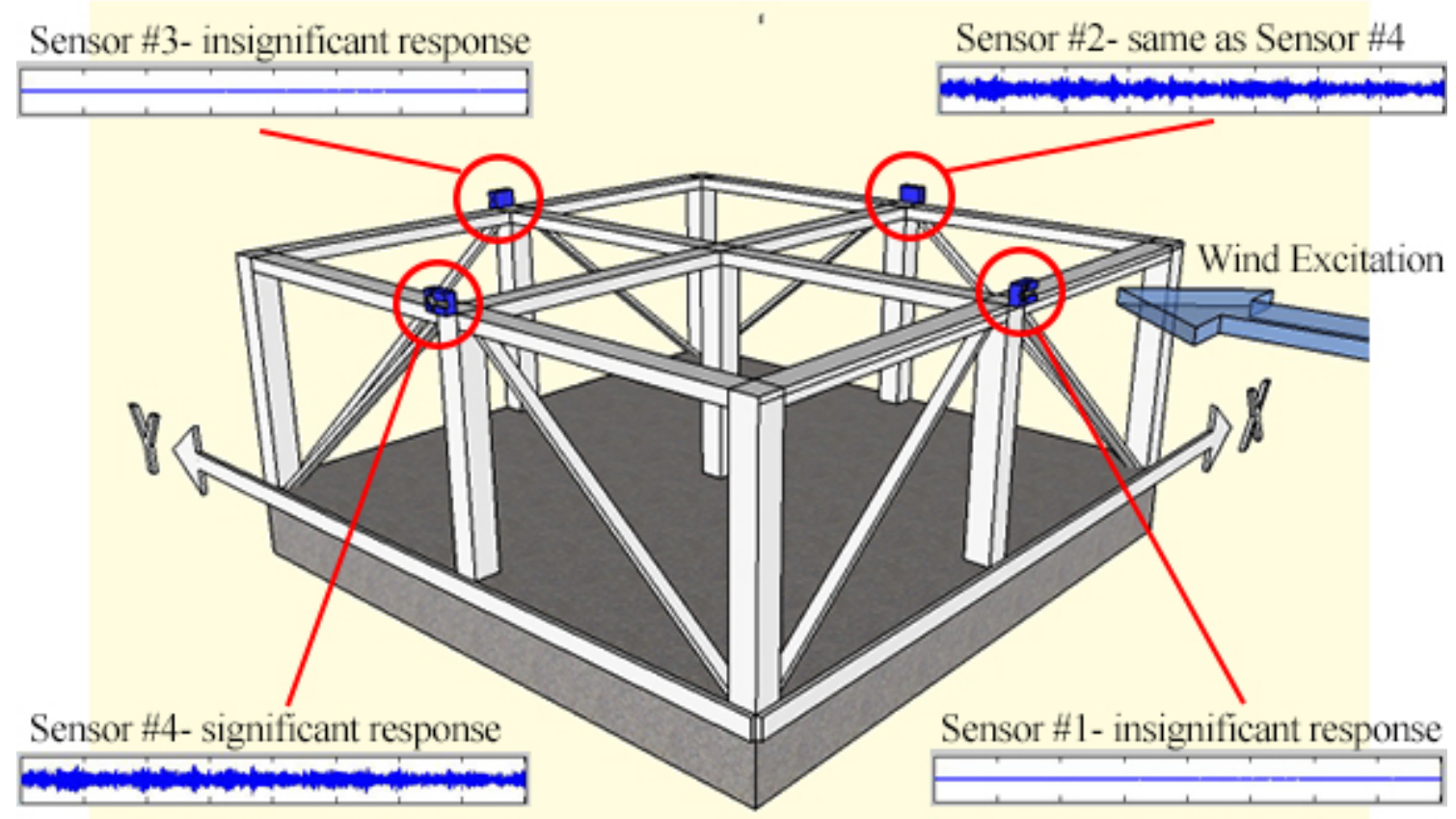

Figure 7.2: Diagram of sensor reduction on one floor

\subsubsection{Damage Matrix Parsing}

Each Datagen simulation runs for a duration of 1.5 seconds. At a sampling frequency of $1 \mathrm{kHz}$, this equates to 1,500 acceleration points from each sensors. Half of the points corresponds to the structure being undamaged, while the remaining half corresponds to whichever damage pattern is being simulated. All of the data is stored in acc_mer. For training and testing purposes, $a c c \_m e r$ is separated into 3 distinct matrices. The first 500 acceleration data for all simulation runs is stored as acc_und. Acceleration points 501 to 1,000 for all simulation runs is stored as acc_trans. Finally, acceleration 
points 1,001 to 1,500 for all simulation runs are stored in acc_dmg. Figure 7.3 demonstrates this at the single-run level.

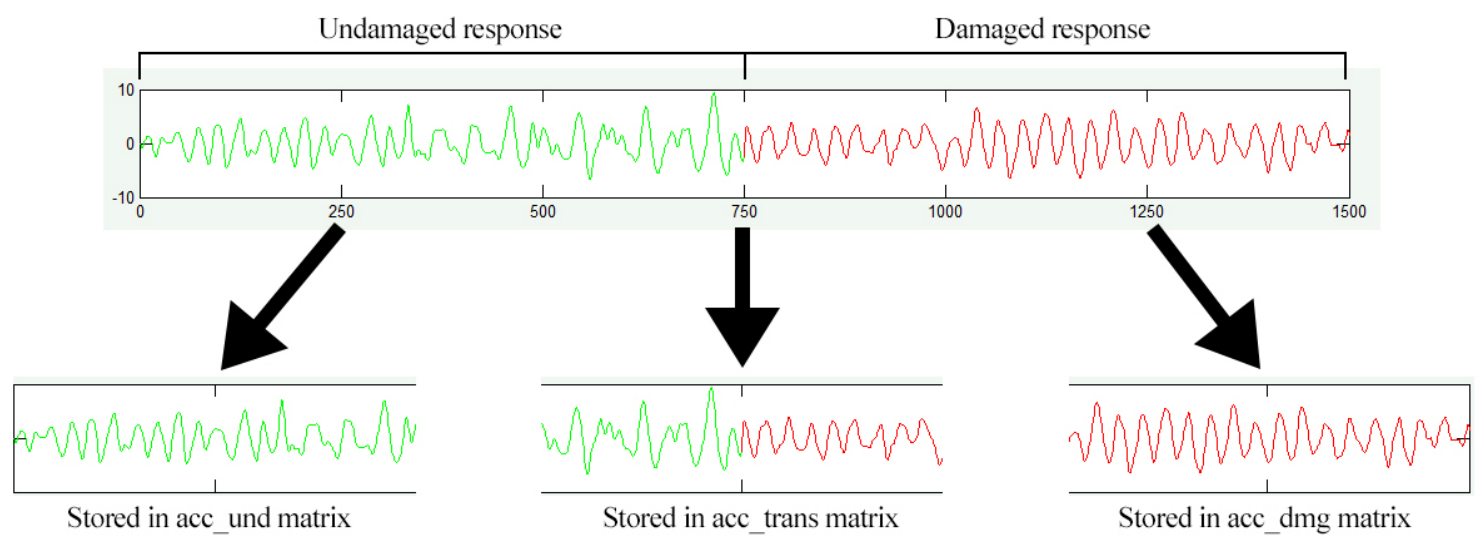

Figure 7.3: Single 1.5 second simulation run parsing example

\subsubsection{Data Generation Output Summary}

Table 7.2 summarizes the final output variables from the data generation stage.

Table 7.2: Data generation output summary

\begin{tabular}{|l|l|}
\hline Variable & Dimensions \\
\hline acc_und & $1 \times 4 \times 200 \times 500$ \\
\hline acc_trans & $9 \times 4 \times 200 \times 500$ \\
\hline acc_dmg & $9 \times 4 \times 200 \times 500$ \\
\hline
\end{tabular}

$\rightarrow$ Raw acceleration data

$\rightarrow$ Dataset

$\rightarrow$ Sensor

$\rightarrow$ Damage patterns

\subsection{Feature Extraction Using Wavelet Transforms}

By modal analysis theory, a structure's parameters change when its integrity changes. The wavelet transform seeks to emphasize these parameter changes before an 
ANN or an AIS algorithm is used to determine the state of the structure. This is done in order to filter out noise and enhance the detail of structural damage.

A wavelet transform contains several parameters which must be set. Many options are tested, but the attempt to optimize the detail enhancement is by no means exhaustive. Parameters are accepted or rejected based upon a preliminary visual inspection using the MATLAB wavelet toolbox. Options which had significant impact upon the coefficients are further investigated. Please see Appendix B for relevant code.

\subsubsection{Mother Wavelet}

A variety of mother wavelets can be used with MATLAB's wavelet toolbox. The Daubechies family is selected and its variations ('haar', db2, db3...db10) are tested. The 'db4' mother wavelet was favorably selected in prior research papers [14] [18] and is thus selected for this thesis.

\subsubsection{Decomposition Level}

Coefficients from detail and approximation levels 1-10 are considered. Approximation level 7 coefficients reliably showed impressive amounts of agitation during and after damage occurs in all damaged cases. Approximation level 7 coefficients of damage pattern 5 can be seen in Figure 7.4, displaying visible effect of structural damage. For the rest of the thesis, wavelet decomposition approximation coefficients at level 7 are used for all simulations. Other levels have also been considered. Please see Appendix A for sample plots. 

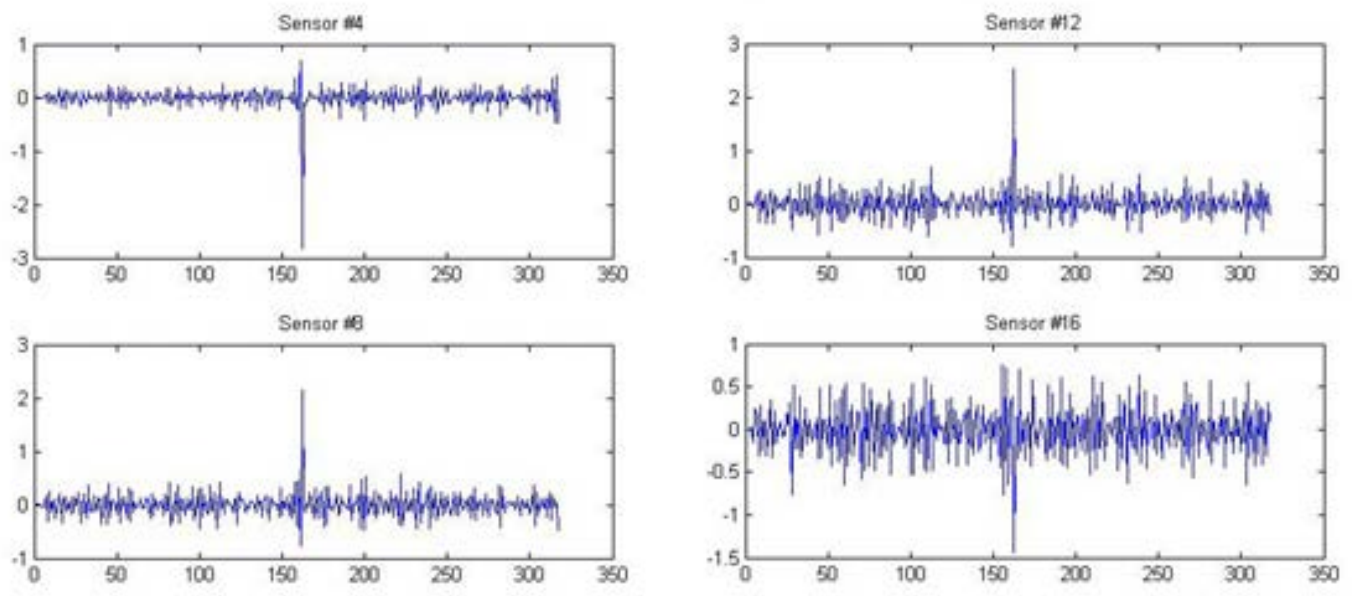

Figure 7.4: CA7 coefficients displaying structural damage

\subsubsection{Edge Padding}

MATLAB offers a variety of edge padding, as described in Chapter 2. All of the options were investigated, and the zero padding 'zpd' option produced coefficients with no edge artifacts. It is therefore used in all wavelet transforms in this thesis.

\subsubsection{Energy Ratio Calculation}

The data generation output matrices acc_und, acc_trans, and acc_dmg are decomposed using the DWT with a 'db4' mother wavelet. The first level detail and approximation coefficients are used in equation (3.10) to calculate the total energy $E_{t}$ for each sample.

Next, using the settings listed in Table 7.3, the DWT decomposes the acc_und, acc_trans, and acc_dmg matrices and outputs the matrices $c A 7 \_u n d, c A 7 \_t r a n s$, and cA7_dmg accordingly. The approximation coefficients of the level 7 decomposition 
yields 10 coefficients per sample. These are used to calculate the specific energy $E_{s}$ for each sample.

Table 7.3: Detail enhancement setup parameters in summary

\begin{tabular}{|l|l|}
\hline Parameter & Value \\
\hline Mother wavelet & $\mathrm{db} 4$ \\
\hline Decomposition level & 7 \\
\hline Level type (detail or approximation) & Approximation \\
\hline Edge padding & zero padding \\
\hline
\end{tabular}

Finally, using equation (3.12), the energy ratio $E_{r}$ is calculated for each sample. These matrices are named $c A 7 \_$undam_E_p, cA7_trans_E_p, and $c A 7 \_d a m \_E \_p$. The names correspond to what damage patterns they represent.

\subsubsection{Feature Extraction Output Summary}

At this stage, the sensor data has been passed through a DWT and each sample's energy ratio is calculated. These matrices are ready to be interpreted using an ANN and AIS.

Table 7.4 summarizes the variables that are output from the detail enhancement stage.

Table 7.4: Detail enhancement stage output

\begin{tabular}{|c|c|}
\hline Variable & Dimensions \\
\hline cA7_undam_E_p & $1 \times 4 \times 200$ \\
\hline cA7_trans_E_p & $9 \times 4 \times 200$ \\
\hline cA7_dam_E_p & $9 \times 4 \times 200$ \\
\hline
\end{tabular}




\subsection{Classification Using ANN}

The acceleration response data generated by Datagen has now been processed through wavelet transforms and is ready to be interpreted using a neural network. Two approaches are tested using neural networks. The first approach uses the neural network to output a Boolean undamaged/damaged value, signifying whether the testing data is damaged or undamaged. The second approach, the multi-damage system, strives to use the neural network to distinguish between undamaged and damaged states, as well as each damage pattern.

In both approaches, the neural network will be trained with half of the available datasets. Once the network is trained, testing data comprising of the remaining unused datasets will be passed through network. The networks will be trained and tested with both transient damage and steady damage data to test its versatility. Native testing signifies that the network is trained and tested with damage data that is from the same category. Non-native testing proves the opposite. Table 7.5 clarifies the possibilities.

Table 7.5: Network testing mode

\begin{tabular}{|l|l|l|}
\hline & $\begin{array}{l}\text { Tested with } \\
\text { transient damage data }\end{array}$ & $\begin{array}{l}\text { Tested with } \\
\text { steady damage data }\end{array}$ \\
\hline $\begin{array}{l}\text { Trained with } \\
\text { transient damage data }\end{array}$ & Native testing & Non-native testing \\
\hline $\begin{array}{l}\text { Trained with } \\
\text { steady damage data }\end{array}$ & Non-native testing & Native testing \\
\hline
\end{tabular}

The network output is then passed through a threshold filter $T(x)$ with a numerical threshold $n$ such that: 


$$
T(x)= \begin{cases}0, & x \leq n \\ 1, & x>n\end{cases}
$$

This output is then compared with the theoretical output to calculate various performance metrics. Figure 7.5 visualizes the process.

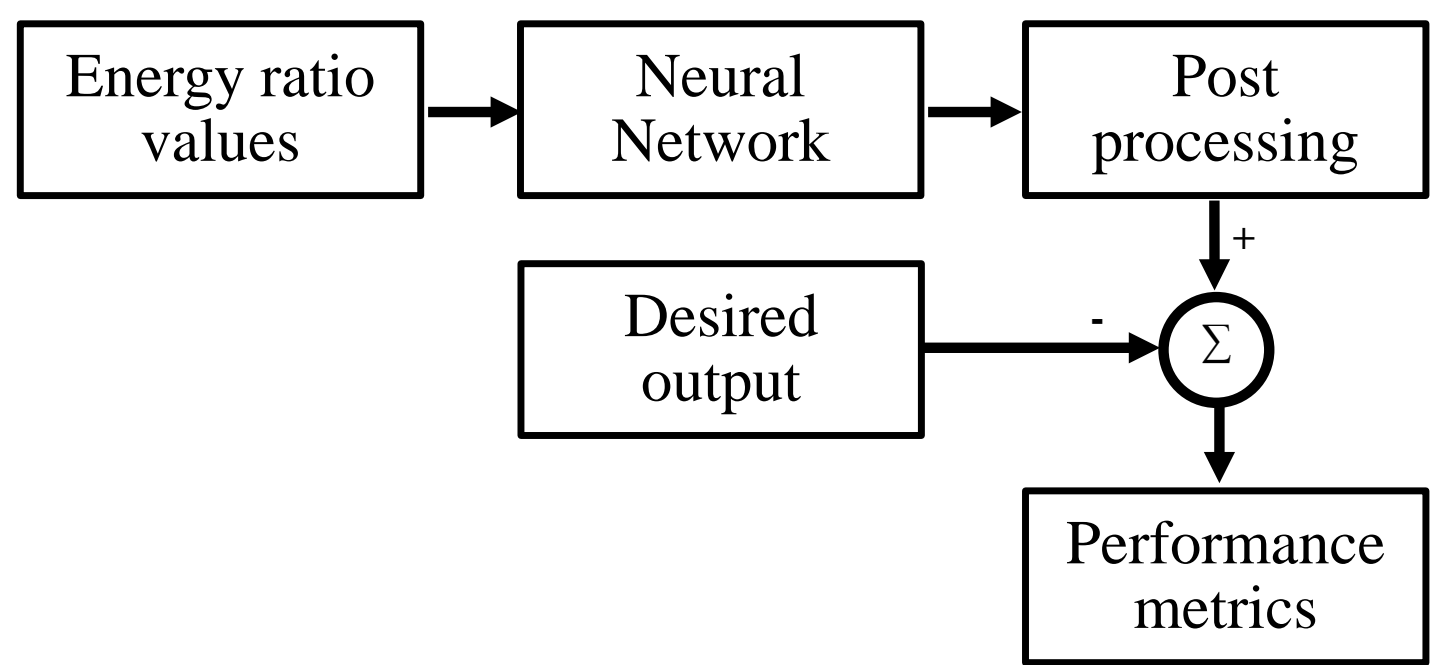

Figure 7.5: Neural network classification workflow

\subsubsection{Approach One- Boolean System}

Training and testing data matrices are prepared for the ANN. The network is set up such that each of the 4 inputs in the input layer corresponds to one of the sensors. The network requires undamaged and damaged training data, as well as training and testing outputs. The training data is extracted either from the $c A 7 \_t r a n s \_E \_p$ or the $c A 7 \_d a m \_E \_p$ matrix. Because of the way these matrices are set up, they both have undamaged data required to train a neural network built into them. Out of the 200 datasets available in each matrix, the first 100 are used for training, and the subsequent 100 are used for testing. Since there are 9 damage patterns including the undamaged patterns, 900 datasets are available for training, and 900 are used for testing. The training 
matrix ANN_train_in_trans is created by extracting and stacking the first 100 datasets of each damage pattern from the $c A 7 \_t r a n s \_E \_p$ matrix. Similarly, the training matrix ANN_train_in_dam is created using the $c A 7 \_d a m \_E \_p$ matrix. Each of these training matrices have dimensions 4x900.

Testing matrices are made using the 100 remaining datasets. ANN_test_in_trans represents the transient damage, while $A N N \_t e s t$ in_dam represents the steady state damage. Each of testing matrices are set up in a similar fashion to their training counterparts and have dimensions 4x900.

A Boolean output matrix named ANN_out_bool is created to house the outputs. This matrix is used both as training and testing output verification and has the dimensions 1x900 doubles. It is set up such that values [1 100] is zero, while [101 900] is one.

\section{Training With Steady Damage Data}

A neural network named net_b1 is generated using MATLAB’s newff command. It contains 4 neurons in the input layer, 60 and 30 neurons in the 2 hidden layers, and 1 neuron in the output layer. The transfer functions used in the 2 hidden layers and the output layer is 'tansig', 'tansig', and 'purelin'. Each training epoch consists of inputting all training data in a random order before updating neuron weights via back-propagation. using the Levenberg-Marquardt optimization. Table 7.6 summarizes the configuration and training parameters for the neural network. 
Table 7.6: Neural network net_b1's configuration and training

\begin{tabular}{|l|l|}
\hline Parameter & Setting \\
\hline Number of hidden layers & 2 \\
\hline Number of neurons per layer & 460301 \\
\hline Transfer function & Tansig tansig purelin \\
\hline Learning method & $\begin{array}{l}\text { Back-propagation using Levenberg- } \\
\text { Marquardt optimization }\end{array}$ \\
\hline Training epochs & 1,000 \\
\hline
\end{tabular}

The training matrix ANN_train_in_dam and ANN_out_bool is used to train network net_b1. After 1,000 training epochs, net_b1 achieved a MSE of 3.98e-06. See Appendix A for a plot of net_b1's MSE.

Once it is trained, the net_b1 is tested with ANN_test_in_trans, which represents transient damage data. Since this is not the native damage category that was used to train the network, more error is expected. The output is passed through a threshold filter of 0.5 , and the results are compared to the expected results matrix ANN_out_bool. Damage specific performance is recorded, while general performance metrics are calculated according to Table 7.7. Table 7.8 details the network's performance, and Table 7.9 summarizes the results.

Table 7.7: General performance metrics calculation equations

\begin{tabular}{|c|c|c|}
\hline & Classified undamaged & Classified damaged \\
\hline $\begin{array}{c}\text { Actual } \\
\text { undamaged }\end{array}$ & $\frac{\text { detected undamaged given undamaged data }}{\text { total undamaged data }}$ & $\frac{\text { detected damaged given undamaged data }}{\text { total undamaged data }}$ \\
\hline $\begin{array}{c}\text { Actual } \\
\text { damaged }\end{array}$ & $\frac{\text { detected undamaged given damaged data }}{\text { total damaged data }}$ & $\frac{\text { detected damaged given damaged data }}{\text { total damaged data }}$ \\
\hline
\end{tabular}

The network is also tested with $A N N \_t e s t \_i n \_d a m$, which represents the same category of data it was trained with. The output is passed through a threshold filter of 0.5 , 
and the results are compared with the expected results matrix ANN_out_bool. Table 7.10 tabulates net_b1's performance with steady damage data, and Table 7.11 summarizes the results.

Table 7.8: Neural network net_b1 [4,60,30,1] transient results

\begin{tabular}{|c|c|c|}
\hline Damage pattern & $\begin{array}{c}\text { \# Correct } \\
\text { (out of 100) }\end{array}$ & $\begin{array}{c}\text { \# Incorrect } \\
\text { (out of 100) }\end{array}$ \\
\hline 1 (undamaged) & 41 & 59 \\
\hline 2 & 59 & 41 \\
\hline 3 & 55 & 45 \\
\hline 4 & 63 & 37 \\
\hline 5 & 56 & 44 \\
\hline 6 & 58 & 42 \\
\hline 7 & 66 & 34 \\
\hline 8 & 75 & 25 \\
\hline 9 & 61 & 39 \\
\hline
\end{tabular}

Table 7.10: Neural network net_b1 $[4,60,30,1]$ steady damage results

\begin{tabular}{|c|c|c|}
\hline Damage pattern & $\begin{array}{c}\text { \# Correct } \\
\text { (out of 100) }\end{array}$ & $\begin{array}{c}\text { \# Incorrect } \\
\text { (out of 100) }\end{array}$ \\
\hline 1 (undamaged) & 81 & 19 \\
\hline 2 & 91 & 9 \\
\hline 3 & 92 & 8 \\
\hline 4 & 92 & 8 \\
\hline 5 & 89 & 11 \\
\hline 6 & 92 & 8 \\
\hline 7 & 90 & 10 \\
\hline 8 & 90 & 10 \\
\hline 9 & 91 & 9 \\
\hline
\end{tabular}

Table 7.9: Neural network net_b1 [4,60,30,1] transient summary

\begin{tabular}{|c|c|c|}
\hline & $\begin{array}{c}\text { Classified } \\
\text { undamaged }\end{array}$ & $\begin{array}{c}\text { Classified } \\
\text { damaged }\end{array}$ \\
\hline $\begin{array}{c}\text { Actual } \\
\text { undamaged }\end{array}$ & $41 \%$ & $59 \%$ \\
\hline $\begin{array}{c}\text { Actual } \\
\text { damaged }\end{array}$ & $38.38 \%$ & $61.62 \%$ \\
\hline
\end{tabular}

Table 7.11: Neural network net_b1 [4,60,30,1] steady damage summary

\begin{tabular}{|c|c|c|}
\hline & $\begin{array}{c}\text { Classified } \\
\text { undamaged }\end{array}$ & $\begin{array}{c}\text { Classified } \\
\text { damaged }\end{array}$ \\
\hline $\begin{array}{c}\text { Actual } \\
\text { undamaged }\end{array}$ & $81 \%$ & $19 \%$ \\
\hline $\begin{array}{c}\text { Actual } \\
\text { damaged }\end{array}$ & $9.13 \%$ & $90.87 \%$ \\
\hline
\end{tabular}

This is an example calculation for classified damage vs. actual damage for Table 7.9:

Based on Table 7.7, the equation is:

$$
\begin{gathered}
\frac{\text { detected damaged given damaged data }}{\text { total damaged data }} \\
=\frac{59+55+63+56+58+66+75+61}{100 \cdot 8}=\frac{493}{800}=0.6162=61.62 \%
\end{gathered}
$$




\section{Training With Transient Damage Data}

A neural network named net_b2 is created using MATLAB's newff command. It is set up similarly to net_b1 (see Table 7.6 for a parameter summary). In this case, net_b2 is trained with $A N N \_t r a i n \_i n \_t r a n s$, which is transient damage data. After 1,000 training epochs, net_b2 achieved a MSE of 7.704e-07. See appendix A for a plot of net_b2's MSE as it is trained.

Once trained, the network net_b2 is tested with ANN_test_in_trans, which represents transient damage data. The output is passed through a threshold filter of 0.5 , and the results are compared to the expected results. Table 7.12 details net_b2's performance, and Table 7.13 summarizes the results.

Table 7.12: Neural network net_b2 [4,60,30,1] transient results

\begin{tabular}{|c|c|c|}
\hline Damage pattern & $\begin{array}{c}\text { \# Correct } \\
\text { (out of 100) }\end{array}$ & $\begin{array}{c}\text { \# Incorrect } \\
\text { (out of 100) }\end{array}$ \\
\hline 1 (undamaged) & 80 & 20 \\
\hline 2 & 98 & 2 \\
\hline 3 & 96 & 4 \\
\hline 4 & 92 & 8 \\
\hline 5 & 89 & 11 \\
\hline 6 & 89 & 11 \\
\hline 7 & 93 & 7 \\
\hline 8 & 95 & 5 \\
\hline 9 & 94 & 6 \\
\hline
\end{tabular}

Table 7.13: Neural network net_b2 [4,60,30,1] transient summary

\begin{tabular}{|c|c|c|}
\hline & $\begin{array}{c}\text { Classified } \\
\text { undamaged }\end{array}$ & $\begin{array}{c}\text { Classified } \\
\text { damaged }\end{array}$ \\
\hline $\begin{array}{c}\text { Actual } \\
\text { undamaged }\end{array}$ & $80 \%$ & $20 \%$ \\
\hline $\begin{array}{c}\text { Actual } \\
\text { damaged }\end{array}$ & $6.75 \%$ & $93.25 \%$ \\
\hline
\end{tabular}

Table 7.14: Neural network net_b2 [4,60,30,1] steady damage results

\begin{tabular}{|c|c|c|}
\hline Damage pattern & $\begin{array}{c}\text { \# Correct } \\
\text { (out of 100) }\end{array}$ & $\begin{array}{c}\text { \# Incorrect } \\
\text { (out of 100) }\end{array}$ \\
\hline 1 (undamaged) & 21 & 79 \\
\hline 2 & 80 & 20 \\
\hline 3 & 77 & 23 \\
\hline 4 & 79 & 21 \\
\hline 5 & 67 & 33 \\
\hline 6 & 66 & 34 \\
\hline 7 & 63 & 37 \\
\hline 8 & 67 & 33 \\
\hline 9 & 68 & 32 \\
\hline
\end{tabular}

Table 7.15: Neural network net_b2 [4,60,30,1] steady damage summary

\begin{tabular}{|c|c|c|}
\hline & $\begin{array}{c}\text { Classified } \\
\text { undamaged }\end{array}$ & $\begin{array}{c}\text { Classified } \\
\text { damaged }\end{array}$ \\
\hline $\begin{array}{c}\text { Actual } \\
\text { undamaged }\end{array}$ & $21 \%$ & $79 \%$ \\
\hline $\begin{array}{c}\text { Actual } \\
\text { damaged }\end{array}$ & $29.13 \%$ & $70.87 \%$ \\
\hline
\end{tabular}


To test the network's robustness, net_b2 is tested with ANN_test_in_dam, which represents steady damage data. Since this damage data is considered a non-native category than what the network is originally trained with, a larger degree of error is expected. The output is passed through a threshold filter of 0.5 , and the results are compared to the expected output. Table 7.14 details net_b2's performance classifying steady damage data, and Table 7.15 summarizes its effectiveness.

\section{Using More Neurons}

A simple test is conducted to investigate whether more neurons structured in a different fashion would result in higher accuracy. In this test, a neural network named net_b1a is created. It contains three hidden layers of 30, 60, and 60 neurons each. Since the output is still a single Boolean value, the output layer remains with one neuron. Table 7.16 summarizes net_b1a's parameters.

Table 7.16: Neural network net_b1a's setup parameters

\begin{tabular}{|l|l|}
\hline Parameter & Setting \\
\hline Number of hidden layers & 3 \\
\hline Number of neurons per layer & 43060601 \\
\hline Transfer function & tansig tansig tansig purelin \\
\hline Learning method & $\begin{array}{l}\text { Back-propagation using Levenberg- } \\
\text { Marquardt optimization }\end{array}$ \\
\hline Training epochs & 1,000 \\
\hline
\end{tabular}

net_b1a uses the same training and testing criteria as net_b1. The only difference in the simulation is the increase of neurons and hidden layers. When trained with ANN_train_in_dam, the network reached a MSE of 3.82e-028 over 1,000 epochs. See Appendix A for a plot of net_b1a's MSE as it is trained. 
The network is then tested with ANN_test_in_trans and ANN_test_in_dam. Both resulting outputs are passed through a threshold filter of 0.5 and compared with the expected results matrix ANN_out_bool. Table 7.17 details net_b1a's performance with transient damage data, and Table 7.18 summarizes the results. Likewise, Table 7.19 details net_b1a's performance with steady damage data, and Table 7.20 summarizes the results.

Table 7.17: Neural network net_b1a $[4,30,60,60,1]$ transient results

\begin{tabular}{|c|c|c|}
\hline Damage pattern & $\begin{array}{c}\text { \# Correct } \\
\text { (out of 100) }\end{array}$ & $\begin{array}{c}\text { \# Incorrect } \\
\text { (out of 100) }\end{array}$ \\
\hline 1 (undamaged) & 44 & 56 \\
\hline 2 & 76 & 24 \\
\hline 3 & 70 & 30 \\
\hline 4 & 80 & 20 \\
\hline 5 & 72 & 28 \\
\hline 6 & 64 & 36 \\
\hline 7 & 74 & 26 \\
\hline 8 & 66 & 34 \\
\hline 9 & 66 & 34 \\
\hline
\end{tabular}

Table 7.18: Neural network net_b1a $[4,30,60,60,1]$ transient summary

\begin{tabular}{|c|c|c|}
\hline & $\begin{array}{c}\text { Classified } \\
\text { undamaged }\end{array}$ & $\begin{array}{c}\text { Classified } \\
\text { damaged }\end{array}$ \\
\hline $\begin{array}{c}\text { Actual } \\
\text { undamaged }\end{array}$ & $44 \%$ & $56 \%$ \\
\hline $\begin{array}{c}\text { Actual } \\
\text { damaged }\end{array}$ & $29 \%$ & $71 \%$ \\
\hline
\end{tabular}

Table 7.19: Neural network net_b1a $[4,30,60,60,1]$ steady damage results

\begin{tabular}{|c|c|c|}
\hline Damage pattern & $\begin{array}{l}\text { \# Correct } \\
\text { (out of 100) }\end{array}$ & $\begin{array}{l}\text { \# Incorrect } \\
\text { (out of 100) }\end{array}$ \\
\hline 1 (undamaged) & 86 & 14 \\
\hline 2 & 97 & 3 \\
\hline 3 & 92 & 8 \\
\hline 4 & 90 & 10 \\
\hline 5 & 91 & 9 \\
\hline 6 & 93 & 9 \\
\hline 7 & 98 & 2 \\
\hline 8 & 92 & 8 \\
\hline 9 & 94 & 6 \\
\hline
\end{tabular}

Table 7.20: Neural network net_b1a $[4,30,60,60,1]$ steady damage summary

\begin{tabular}{|c|c|c|}
\hline & $\begin{array}{c}\text { Classified } \\
\text { undamaged }\end{array}$ & $\begin{array}{c}\text { Classified } \\
\text { damaged }\end{array}$ \\
\hline $\begin{array}{c}\text { Actual } \\
\text { undamaged }\end{array}$ & $86 \%$ & $14 \%$ \\
\hline $\begin{array}{c}\text { Actual } \\
\text { damaged }\end{array}$ & $6.63 \%$ & $93.37 \%$ \\
\hline
\end{tabular}

\subsubsection{Approach Two- Multi-damage System.}

In this approach, the neural network is set up to distinguish between each damage pattern. This is more difficult than simply distinguishing between undamaged and damaged. Training and testing input matrices are prepared for the ANN in the same 
fashion as in section 7.3.1 above. The matrices ANN_train_in_trans and ANN_train_in_dam serve as training inputs to the neural networks, while ANN_test_in_trans and ANN_test_in_dam serve as testing matrices for trained networks. They still have dimensions 4x900.

A matrix named ANN_out_1shot is created for training and testing output verification. This 9x900 matrix is first created with zeroes. Then, [2, 101:200], [3, 201:300], [4, 301:400], [5, 401:500], [6, 501:600], [7, 601:700], [8, 701:800], and [9, 801:900] are set as one. This corresponds to the input matrices such that each output is mapped to a specific damage pattern. Output setups like this are often called one-shot outputs. Table 7.21 demonstrates the output expected by ANN_out_1shot.

Table 7.21: Neural network one-shot output setup

\begin{tabular}{|l|l|}
\hline Damage pattern & Expected network output \\
\hline 1 (undamaged) & 000000000 \\
\hline 2 & 000000010 \\
\hline 3 & 000000100 \\
\hline 4 & 000001000 \\
\hline 5 & 000010000 \\
\hline 6 & 000100000 \\
\hline 7 & 001000000 \\
\hline 8 & 010000000 \\
\hline 9 & 100000000 \\
\hline
\end{tabular}

\section{Training With Steady Damaged Data}

A neural network named net_1shot_1 is created using MATLAB's newff command. It contains 4 neurons in the input layer, 80 and 60 neurons in the 2 hidden layers, and 9 neurons in the output layer. The transfer functions used in the 2 hidden layers and the output layer is 'tansig', 'tansig', and 'purelin'. Weight and bias values are 
updated via back-propagation via the Levenberg-Marquardt optimization. Table 7.22 summarizes the setup parameters for the neural network net_1shot_1.

Table 7.22: Neural network net_1shot_1's setup parameters

\begin{tabular}{|l|l|}
\hline Parameter & Setting \\
\hline Number of hidden layers & 2 \\
\hline Number of neurons per layer & 480609 \\
\hline Transfer function & tansig tansig purelin \\
\hline Learning method & $\begin{array}{l}\text { Back-propagation using Levenberg- } \\
\text { Marquardt optimization }\end{array}$ \\
\hline Training epochs & 1,000 \\
\hline
\end{tabular}

The network net_1shot_1 is trained with ANN_train_in_dam, which represents steady damage data. The expected output matrix used is ANN_out_1shot. After 1,000 training epochs, net_1shot_1 reached a MSE of 1.38e-02. See Appendix A for a plot of net_1shot_1's MSE as it is trained.

Once trained, net_1shot_1 is first tested with transient damage data using ANN_test_out_trans. Since this is not the native damage category as the data the network is trained with, higher error is expected. The output is passed through a threshold filter of 0.5 , and the results are compared with the expected output matrix ANN_out_1shot. See Appendix A for a full confusion matrix. Table 7.23 tabulates the individual percent correct (true positive) and incorrect (false positive) of each damage pattern, while Table 7.24 summarizes the general results.

Similarly, net_1shot_1 is tested with steady damage data using ANN_test_out_dam. The output is passed through a threshold filter of 0.5 , and the results are compared with the expected output matrix ANN_out_1shot. See Appendix A for a full confusion matrix. Table 7.25 tabulates the individual percent correct (true positive) 
and incorrect (false positive) of each damage pattern, while Table 7.26 summarizes the results.

It should be noted that for each neural network, the first table tabulates the percent error for each damage pattern, while the second summary table summarizes the general performance calculated according to Table 7.7. This is relevant in this approach because each damage classification whether correct or incorrect is considered as a damaged. Here is an example of how the classified undamaged vs. actual damaged percentage in Table 7.24. is calculated. The raw data used is available in Appendix A.

Based on Table 7.7, the equation is:

$$
\begin{gathered}
\frac{\text { detected undamaged given damaged data }}{\text { total damaged data }} \\
=\frac{4.93+6.15+12.09+8.36+7.87+12.59+9.7+9.27}{100 \cdot 8}=\frac{70.96}{800}=0.0887=8.87 \%
\end{gathered}
$$

Table 7.23: Neural network net_1shot_1 $[4,80,60,9]$ transient results

\begin{tabular}{|c|c|c|}
\hline Damage pattern & $\begin{array}{c}\text { \% Correct } \\
\text { (out of 100) }\end{array}$ & $\begin{array}{c}\text { \% Incorrect } \\
\text { (out of 100) }\end{array}$ \\
\hline 1 (undamaged) & 100.00 & 0.00 \\
\hline 2 & 16.14 & 83.86 \\
\hline 3 & 31.43 & 68.57 \\
\hline 4 & 10.16 & 89.84 \\
\hline 5 & 11.76 & 88.24 \\
\hline 6 & 11.95 & 88.05 \\
\hline 7 & 10.14 & 89.86 \\
\hline 8 & 12.12 & 87.88 \\
\hline 9 & 13.10 & 86.90 \\
\hline
\end{tabular}

Table 7.25: Neural network net_1shot_1 $[4,80,60,9]$ steady damage results

\begin{tabular}{|c|c|c|}
\hline Damage pattern & $\begin{array}{c}\text { \% Correct } \\
\text { (out of 100) }\end{array}$ & $\begin{array}{c}\text { \% Incorrect } \\
\text { (out of 100) }\end{array}$ \\
\hline 1 (undamaged) & 100 & 0 \\
\hline 2 & 64.10 & 35.90 \\
\hline 3 & 78.57 & 21.43 \\
\hline 4 & 46.54 & 53.46 \\
\hline 5 & 41.44 & 58.56 \\
\hline 6 & 46.11 & 53.89 \\
\hline 7 & 49.01 & 50.99 \\
\hline 8 & 49.03 & 50.97 \\
\hline 9 & 50.68 & 49.32 \\
\hline
\end{tabular}


Table 7.24: Neural network net_1shot_1 $[4,80,60,9]$ transient summary

\begin{tabular}{|l|l|l|}
\hline & $\begin{array}{l}\text { Classified } \\
\text { undamaged }\end{array}$ & $\begin{array}{l}\text { Classified } \\
\text { damaged }\end{array}$ \\
\hline $\begin{array}{l}\text { Actual } \\
\text { undamaged }\end{array}$ & $100 \%$ & $0 \%$ \\
\hline $\begin{array}{l}\text { Actual } \\
\text { damaged }\end{array}$ & $8.87 \%$ & $91.13 \%$ \\
\hline
\end{tabular}

Table 7.26: Neural network net_1shot_1 $[4,80,60,9]$ steady damage summary

\begin{tabular}{|c|c|c|}
\hline & $\begin{array}{c}\text { Classified } \\
\text { undamaged }\end{array}$ & $\begin{array}{c}\text { Classified } \\
\text { damaged }\end{array}$ \\
\hline $\begin{array}{c}\text { Actual } \\
\text { undamaged }\end{array}$ & $100 \%$ & $0 \%$ \\
\hline $\begin{array}{c}\text { Actual } \\
\text { damaged }\end{array}$ & $6.15 \%$ & $93.85 \%$ \\
\hline
\end{tabular}

\section{Training With Transient Damage Data}

A neural network named net_1shot_2 is created using MATLAB's newff command. It is set up similarly to net_1shot_1 above (see Table 7.22 for a summary). The major difference between the two networks is that net_1shot_2 will be trained with transient damage data instead of steady damage data.

The network net_1shot_2 is trained with ANN_train_in_dam, which represents steady damage data. The expected output matrix used is ANN_out_1shot. After 1,000 training epochs, net_1shot_2 reached a MSE of 9.51e-03. See Appendix A for a plot of net_1shot_2's MSE as it is trained. Once trained, net_1shot_2 is first tested with transient damage data using ANN_test_out_trans. The output is passed through a threshold filter of 0.5 , and the results are compared with the expected output matrix ANN_out_1shot. See Appendix A for a full confusion matrix. Table 7.27 tabulates the individual percent correct (true positive) and incorrect (false positive) of each damage pattern, while Table 7.28 summarizes the results.

Similarly, net_1shot_2 is tested with steady damage data using ANN_test_out_dam. Since this is not the native damage category as the data the network is trained with, higher error is expected. The output is passed through a threshold filter of 
0.5 , and the results are compared with the expected output matrix ANN_out_1shot. See Appendix A for a full confusion matrix. Table 7.29 tabulates the individual percent correct (true positive) and incorrect (false positive) of each damage pattern, while Table 7.30 summarizes the results.

Table 7.27: Neural network net_1shot_2 $[4,80,60,9]$ transient results

\begin{tabular}{|c|c|c|}
\hline Damage pattern & $\begin{array}{c}\text { \% Correct } \\
\text { (out of 100) }\end{array}$ & $\begin{array}{c}\text { \% Incorrect } \\
\text { (out of 100) }\end{array}$ \\
\hline 1 (undamaged) & 100.00 & 0.00 \\
\hline 2 & 58.90 & 41.10 \\
\hline 3 & 75.79 & 24.21 \\
\hline 4 & 41.06 & 58.94 \\
\hline 5 & 45.68 & 54.32 \\
\hline 6 & 43.48 & 56.52 \\
\hline 7 & 49.40 & 50.60 \\
\hline 8 & 51.20 & 48.80 \\
\hline 9 & 56.39 & 43.61 \\
\hline
\end{tabular}

Table 7.28: Neural network net_1shot_2 $[4,80,60,9]$ transient summary

\begin{tabular}{|c|c|c|}
\hline & $\begin{array}{c}\text { Classified } \\
\text { undamaged }\end{array}$ & $\begin{array}{c}\text { Classified } \\
\text { damaged }\end{array}$ \\
\hline $\begin{array}{c}\text { Actual } \\
\text { undamaged }\end{array}$ & $100 \%$ & $0 \%$ \\
\hline $\begin{array}{c}\text { Actual } \\
\text { damaged }\end{array}$ & $4.61 \%$ & $95.39 \%$ \\
\hline
\end{tabular}

Table 7.29: Neural network net_1shot_2 $[4,80,60,9]$ steady damage results

\begin{tabular}{|c|c|c|}
\hline Damage pattern & $\begin{array}{c}\text { \% Correct } \\
\text { (out of 100) }\end{array}$ & $\begin{array}{c}\text { \% Incorrect } \\
\text { (out of 100) }\end{array}$ \\
\hline 1 (undamaged) & 100.00 & 0.00 \\
\hline 2 & 18.37 & 81.63 \\
\hline 3 & 36.70 & 63.30 \\
\hline 4 & 11.74 & 88.26 \\
\hline 5 & 10.92 & 89.08 \\
\hline 6 & 10.77 & 89.23 \\
\hline 7 & 15.45 & 84.55 \\
\hline 8 & 13.21 & 86.79 \\
\hline 9 & 10.20 & 89.8 \\
\hline
\end{tabular}

Table 7.30: Neural network net_1shot_2 $[4,80,60,9]$ steady damage summary

\begin{tabular}{|c|c|c|}
\hline & $\begin{array}{c}\text { Classified } \\
\text { undamaged }\end{array}$ & $\begin{array}{c}\text { Classified } \\
\text { damaged }\end{array}$ \\
\hline $\begin{array}{c}\text { Actual } \\
\text { undamaged }\end{array}$ & $100 \%$ & $0 \%$ \\
\hline $\begin{array}{c}\text { Actual } \\
\text { damaged }\end{array}$ & $10.06 \%$ & $89.94 \%$ \\
\hline
\end{tabular}

\section{Increasing Training Epochs}

A simple trial is conducted to see if increasing the training time would yield better results. A variant of net_1shot_1, called net_1shot_1a is created for this purpose. Its training and setup parameters are exactly the same as net_1shot_1 (see Table 7.22 for a summary), except it is allowed to train up to 2,000 epochs instead of 1,000 . 
The network net_1shot_1a is trained with steady damage data from the matrix ANN_train_in_dam. The output used is ANN_out_1shot. After 2,000 training epochs, the network achieved a MSE of 7.67e-03. See Appendix A for a plot of net_1shot_1a's MSE as it is trained.

Once trained, net_1shot_1a is first tested with transient damage data using ANN_test_out_trans. This is not the native damage category as the data the network is trained with, so higher error is expected. The output is passed through a threshold filter of 0.5 , and the results are compared with the expected output matrix ANN_out_1shot. See Appendix A for a full confusion matrix. Table 7.31 tabulates the individual percent correct (true positive) and incorrect (false positive) of each damage pattern, while Table 7.32 summarizes the results.

Similarly, net_1shot_1a is tested with steady damage data using ANN_test_out_dam. The output is passed through a threshold filter of 0.5, and the results are compared with the expected output matrix ANN_out_1shot. See Appendix A for a full confusion matrix. Table 7.33 tabulates the individual percent correct (true positive) and incorrect (false positive) of each damage pattern, while Table 7.34 summarizes the results. 
Table 7.31: Neural network net_1shot_1a $[4,80,60,9]$ transient results

\begin{tabular}{|c|c|c|}
\hline Damage pattern & $\begin{array}{c}\text { \% Correct } \\
\text { (out of 100) }\end{array}$ & $\begin{array}{c}\text { \% Incorrect } \\
\text { (out of 100) }\end{array}$ \\
\hline 1 (undamaged) & 100.00 & 0.00 \\
\hline 2 & 17.68 & 82.32 \\
\hline 3 & 32.86 & 67.14 \\
\hline 4 & 12.43 & 87.57 \\
\hline 5 & 12.35 & 87.65 \\
\hline 6 & 9.64 & 90.36 \\
\hline 7 & 9.94 & 90.06 \\
\hline 8 & 14.97 & 85.03 \\
\hline 9 & 8.82 & 91.18 \\
\hline
\end{tabular}

Table 7.32: Neural network net_1shot_1a $[4,80,60,9]$ transient summary

\begin{tabular}{|c|c|c|}
\hline & $\begin{array}{c}\text { Classified } \\
\text { undamaged }\end{array}$ & $\begin{array}{c}\text { Classified } \\
\text { damaged }\end{array}$ \\
\hline $\begin{array}{c}\text { Actual } \\
\text { undamaged }\end{array}$ & $100 \%$ & $0 \%$ \\
\hline $\begin{array}{c}\text { Actual } \\
\text { damaged }\end{array}$ & $9.54 \%$ & $90.46 \%$ \\
\hline
\end{tabular}

Table 7.33: Neural network net_1shot_1a $[4,80,60,9]$ steady damage results

\begin{tabular}{|c|c|c|}
\hline Damage pattern & $\begin{array}{c}\text { \% Correct } \\
\text { (out of 100) }\end{array}$ & $\begin{array}{c}\text { \% Incorrect } \\
\text { (out of 100) }\end{array}$ \\
\hline 1 (undamaged) & 100.00 & 0.00 \\
\hline 2 & 70.65 & 29.35 \\
\hline 3 & 80.81 & 19.19 \\
\hline 4 & 40.86 & 59.14 \\
\hline 5 & 44.00 & 56.00 \\
\hline 6 & 46.02 & 53.98 \\
\hline 7 & 46.71 & 53.29 \\
\hline 8 & 48.77 & 51.23 \\
\hline 9 & 43.78 & 56.22 \\
\hline
\end{tabular}

Table 7.34: Neural network net_1shot_1a $[4,80,60,9]$ steady damage summary

\begin{tabular}{|c|c|c|}
\hline & $\begin{array}{c}\text { Classified } \\
\text { undamaged }\end{array}$ & $\begin{array}{c}\text { Classified } \\
\text { damaged }\end{array}$ \\
\hline $\begin{array}{c}\text { Actual } \\
\text { undamaged }\end{array}$ & $100 \%$ & $0 \%$ \\
\hline $\begin{array}{c}\text { Actual } \\
\text { damaged }\end{array}$ & $5.96 \%$ & $94.04 \%$ \\
\hline
\end{tabular}

\subsubsection{Summary}

\section{Approach One- Boolean System}

Neural networks proved to be proficient in properly identifying the damage category it has been trained with. In the first approach, the neural networks were tasked to identify whether the structure was undamaged or damaged. When tested with native damage data (data which represented the same damage category as its training data, whether it’s transient damage or steady damage), the neural networks averaged $\mathbf{8 2 . 3 3 \%}$ in correctly identifying undamaged data, and $\mathbf{9 2 . 5 0 \%}$ in correctly identifying damaged data. Table 7.35 summarizes the true positive and true negative results. 
Table 7.35: Boolean network average results for native category data

\begin{tabular}{|l|c|c|}
\hline & Correct undamaged & Correct damaged \\
\hline net_b1 & $81 \%$ & $90.87 \%$ \\
\hline net_b1a & $86 \%$ & $93.37 \%$ \\
\hline net_b2 & $80 \%$ & $93.25 \%$ \\
\hline Average & $\mathbf{8 2 . 3 3 \%}$ & $\mathbf{9 2 . 5 0 \%}$ \\
\hline
\end{tabular}

From these results, we can conclude that when tasked to identify between damage and undamaged data, neural networks are effective when detecting damage states in native mode. Within this setup, the network is better at detecting damage patterns than undamaged patterns. In addition, the network trained with transient damage data is able to detect its native damage pattern slightly better than the network trained with steady damage data.

The networks are then tested with non-native data to see how robust it is in identifying cases outside its training data. The network averaged $35.33 \%$ in correctly identifying undamaged patterns, and $\mathbf{6 7 . 8 3 \%}$ in identifying damaged patterns. Table 7.36 summarizes the true positive and true negative results.

Table 7.36: Boolean network average results for non-native category data

\begin{tabular}{|l|c|c|}
\hline & Correct undamaged & Correct damaged \\
\hline net_b1 & $41 \%$ & $61.62 \%$ \\
\hline net_b1a & $44 \%$ & $71 \%$ \\
\hline net_b2 & $21 \%$ & $70.87 \%$ \\
\hline Average & $35.33 \%$ & $\mathbf{6 7 . 8 3 \%}$ \\
\hline
\end{tabular}

The effects of testing the networks with non-native damage data covering out of its training data can be seen in the results. The networks' effectiveness are reduced in detecting both undamaged and damaged states, but the undamaged detection rate suffered a higher accuracy loss. This means that when tested with damage data outside of its 
training data, the neural network will tend to return more false positives than usual. Compared to the network trained with steady damage data, the network trained with transient damage was able to detect non-native damage more accurately $(70.87 \%$ vs. $61.62 \%)$. However, its accuracy in detecting undamaged patterns (21\%) is worse than the network that was trained with steady damage (41\%).

\section{Approach Two- Multi-damage System}

When tasked with identifying each damage pattern individually, neural networks showed promising results. When tested with the native damage data, the networks were able to detect the most severe damage pattern (pattern \#3) with an average accuracy of 78.39\%. The second most severe damage pattern (pattern \#2) was recognized with an average accuracy of $\mathbf{6 4 . 5 5 \%}$. While the networks were not able to distinguish between the other damage patterns with good accuracy, their general capability of distinguishing between undamaged data from damaged data was wholly better than the Boolean networks in approach one. Table 7.37 summarizes the results.

Table 7.37: Multi-damage network average results for native category data

\begin{tabular}{|l|c|c|}
\hline & Correct undamaged & Correct damaged \\
\hline net_1shot_1 & $100 \%$ & $93.85 \%$ \\
\hline net_1shot_1b & $100 \%$ & $94.04 \%$ \\
\hline net_1shot_2 & $100 \%$ & $95.39 \%$ \\
\hline Average & $\mathbf{1 0 0 \%}$ & $\mathbf{9 4 . 4 3 \%}$ \\
\hline
\end{tabular}

These outcomes reveal that setting up the multi-damage networks for detecting native data results in a stellar $\mathbf{1 0 0 \%}$ accuracy in identifying undamaged data. This equates to the network giving no false positive outcomes. In addition, their ability to 
detect damage in general (94.43\%) is better than Boolean networks (92.50\%). For the purpose of detecting native damage, the network trained with transient damage data was able to detect its native damage more accurately than the network trained with steady damage data (95.39\% vs. 93.85\%).

The multi-damage networks are also tasked to identify non-native damage data. This tests how robust the networks are to data that is outside of its standard training inputs. Table 7.38 summarizes these results.

Table 7.38: Multi-damage network average results for non-native category data

\begin{tabular}{|l|c|c|}
\hline & Correct undamaged & Correct damaged \\
\hline net_1shot_1 & $100 \%$ & $91.13 \%$ \\
\hline net_1shot_1b & $100 \%$ & $90.46 \%$ \\
\hline net_1shot_2 & $100 \%$ & $89.94 \%$ \\
\hline Average & $\mathbf{1 0 0 \%}$ & $\mathbf{9 0 . 5 1 \%}$ \\
\hline
\end{tabular}

Even when tested with non-native damage data, the multi-damage networks retain their $\mathbf{1 0 0 \%}$ accuracy in correctly identifying the undamaged state. More importantly, the networks averaged $\mathbf{9 0 . 5 1 \%}$ in identifying damage states that are non-native to them. While this is slightly less than the native detection results (94.43\%), the multi-damage networks fared far better in identifying non-native damage than their Boolean counterparts (67.83\%). For the purpose of identifying non-native data, the network trained with steady damage data fared slightly better than the network trained with transient damage data (91.13\% vs. 89.94\%).

\section{Miscellaneous Improvements}

The experiment in finding the effects from increasing the number of neurons in a neural network yielded positive results. Compared to net_b1, net_b1a's results are 
consistently better (see Table 7.35 and Table 7.36). This signifies that the current neural network could probably benefit from an increase in neurons in hidden layers without any negative consequences.

Testing to see whether increasing the training epochs would improve the neural network’s performance yielded mixed results (see Table 7.37 and Table 7.38). Compared to net_1shot_1, net_1shot_1a yielded marginally better results in detecting native damage, but suffered slightly in detecting non-native damage. This is to be expected, as net_1shot_1a had more training epochs to form to its training data. Over the 2,000 training epochs, its MSE of 7.67e-03 is only slightly better than net_1shot_1's MSE of 1.38e-02 after 1,000 training epochs. More than anything, net_1shot_1a seemed to be constrained primarily by its number of neurons; increasing that instead of training it longer would yield better results.

\subsection{Classification Using AIS}

An overlapping variable-radius, randomly generated NS algorithm is written in MATLAB to process the wavelet coefficient matrices generated in the "detail enhancement” stage. See Appendix B for the MATLAB code. This program is based on the NS algorithm written to demonstrate various coverage techniques in Chapter 5.

The training and testing data first undergo a pre-scaling stage. Next, the NS algorithm will be trained with undamaged "self" data extracted from $c A 7 \_u n d a m \_E \_p$, $c A 7 \_t r a n s \_E \_p$, and $c A 7 \_d a m \_E \_p$ matrices. Renamed as AIS_train, this matrix consists of 600 undamaged datasets. Using this training matrix, the NS algorithm will attempt to 
randomly generate a preset number of detectors, calculating distances using a set n-norm. Detectors with a radii below a threshold are discarded.

Once the generation phase is complete, the NS algorithm will then be tested using datasets extracted from both $c A 7 \_t r a n s \_E \_p$ and $c A 7 \_d a m_{-} E \_p$. Two approaches in applying the NS algorithm will be tested. In the first approach, data from all four sensors are considered. The second approach, however, uses data from only two of the sensors are used. This is an attempt to improve classification by reducing the complexity order.

\subsubsection{Data Pre-scaling}

The negative selection algorithm is set up to operate within a space of [0 1] for each dimension. For that to work, the training and testing data has to be scaled into that range. Both training and testing datasets are scaled using the maximum value of the two. This ensures that the testing and training space remain consistent, and that both datasets are within [0 1 l].

\subsubsection{Preliminary Optimization}

The AIS algorithm offers many parameters which many affect its effectiveness. These include the number of detectors to generate, minimum detector radii, radii overlap percentage, and n-norm for calculating distance. A preliminary survey is done in order to find a semi-optimal value for each parameter. This is accomplished where each variable is swept over a range while the rest remains constant. The random number generator seed is locked to ensure consistency. Undamaged data from $c A 7 \_u n d a m \_E \_p$ is 
used to train the matrix, and a stacked damaged matrix used in the neural network testing in Chapter 7.3.1 is used to test the neural network. See Appendix A for a summary of the survey.

The survey concluded that a $\mathbf{2 , 0 0 0}$ detectors should be generated with a minimum detector radius of $\mathbf{0 . 0 0 0 5}$. A 2-norm metric should be used to calculate the distance. Radius overlap should be set to $\mathbf{5 0 \%}$. Table 7.39 below summarizes the AIS parameters.

Table 7.39: AIS setup parameters summary

\begin{tabular}{|l|l|}
\hline Parameter & Value \\
\hline Number of detectors to generate & 2,000 \\
\hline Minimum detector radius & 0.0005 \\
\hline \% of radius allowed to overlap & $50 \%$ \\
\hline N-norm for distance calculation & 2 \\
\hline
\end{tabular}

\subsubsection{Approach One- Full Sensor Data}

The AIS is trained with the AIS_train matrix using the settings listed in Table 7.39 above. It is tested with AIS_test, which is a $4 \times 400$ matrix consisting of 200 undamaged datasets from $c$ A7_trans_E_p, as well as 200 transient damage datasets of the specific damage pattern being tested, also extracted from $c A 7$ trans_E_p. Both matrices have undergone a preliminary scaling stage. Once the generation and detection phases are complete, the AIS's classifications are compared to the expected results to calculate metrics. The performance summary is calculated according to Table 7.7. Table 7.40 details each damage pattern’s outcome, and Table 7.41 summarizes the results.

Subsequently, the AIS is tested using steady damage datasets. The undamaged training dataset remains as the AIS_train matrix. The 4x400 testing matrix AIS_test now extracts its datasets from the $c A 7 \_d a m_{-} E \_p$ matrix instead. Once again, the first 200 
testing datasets represent the undamaged state, while the latter 200 datasets represent the damage pattern being tested. After the data is passed through a scaling stage, the AIS is trained with AIS_train, then tested with AIS_test. The output is then compared to the expected results. Table 7.42 details the steady damage outcomes, and Table 7.43 summarizes the results.

Table 7.40: Full sensor AIS transient results

\begin{tabular}{|c|c|c|}
\hline Damage pattern & $\begin{array}{c}\text { \% Correct } \\
\text { (out of 100) }\end{array}$ & $\begin{array}{c}\text { \% Incorrect } \\
\text { (out of 100) }\end{array}$ \\
\hline 1 (undamaged) & 100.00 & 0.00 \\
\hline 2 & 67.5 & 32.5 \\
\hline 3 & 82.5 & 17.5 \\
\hline 4 & 62 & 38 \\
\hline 5 & 54.5 & 45.5 \\
\hline 6 & 57 & 43 \\
\hline 7 & 36.5 & 63.5 \\
\hline 8 & 61.5 & 38.5 \\
\hline 9 & 56 & 44 \\
\hline
\end{tabular}

Table 7.41: Full sensor AIS transient summary

\begin{tabular}{|c|c|c|}
\hline & $\begin{array}{c}\text { Classified } \\
\text { undamaged }\end{array}$ & $\begin{array}{c}\text { Classified } \\
\text { damaged }\end{array}$ \\
\hline $\begin{array}{c}\text { Actual } \\
\text { undamaged }\end{array}$ & $100 \%$ & $0 \%$ \\
\hline $\begin{array}{c}\text { Actual } \\
\text { damaged }\end{array}$ & $40.31 \%$ & $59.69 \%$ \\
\hline
\end{tabular}

Table 7.42: Full sensor AIS steady damage results

\begin{tabular}{|c|c|c|}
\hline Damage pattern & $\begin{array}{c}\text { \% Correct } \\
\text { (out of 100) }\end{array}$ & $\begin{array}{c}\text { \% Incorrect } \\
\text { (out of 100) }\end{array}$ \\
\hline 1 (undamaged) & 100.00 & 0.00 \\
\hline 2 & 72 & 28 \\
\hline 3 & 66 & 34 \\
\hline 4 & 35.5 & 64.5 \\
\hline 5 & 54.5 & 45.5 \\
\hline 6 & 46.5 & 53.5 \\
\hline 7 & 47 & 53 \\
\hline 8 & 43.5 & 56.5 \\
\hline 9 & 33.5 & 66.5 \\
\hline
\end{tabular}

Table 7.43: Full sensor AIS steady damage summary

\begin{tabular}{|l|l|l|}
\hline & $\begin{array}{l}\text { Classified } \\
\text { undamaged }\end{array}$ & $\begin{array}{l}\text { Classified } \\
\text { damaged }\end{array}$ \\
\hline $\begin{array}{l}\text { Actual } \\
\text { undamaged }\end{array}$ & $100 \%$ & $0 \%$ \\
\hline $\begin{array}{l}\text { Actual } \\
\text { damaged }\end{array}$ & $49.81 \%$ & $50.19 \%$ \\
\hline
\end{tabular}

\subsubsection{Approach Two- Dual Sensor Data}

As the number of input increases, the dimensionality also increases. This increases the complexity of the non-self coverage exponentially. In approach one where the AIS accepts four inputs, the non-self space it attempts to cover with 2,000 detectors is 
simply inadequate. As a result, the algorithm returns many false negatives. An attempt to rectify this issue is presented in approach two.

In this approach, the AIS is set up such that its input complexity is reduced to two. This is accomplished by utilizing only the first and fourth floor sensor data coefficient energy ratios instead of every floors' data. The 2x600 training matrix AIS_train contains 600 undamaged state datasets from the first and fourth floor. The 2x400 testing matrix AIS_test contains 200 undamaged datasets as well as 200 damaged datasets. The damaged datasets are extracted from either $c A 7 \_t r a n s \_E \_p$ or $c A 7 \_d a m \_E \_p$, depending upon the damage pattern and damage category (steady or transient damage). AIS_train and AIS_test both undergo a preliminary scaling stage before being utilized by the AIS.

The AIS is still setup with the same parameters listed in Table 7.39, with the addition of a generation phase interrupt. During the generation phase, if the algorithm fails to produce a valid detector after $\mathbf{5 0 , 0 0 0}$ consecutive attempts, the interrupt code ends the generation phase and proceeds to the detection phase. This value ensures that a dominant amount non-self coverage is met within a reasonable amount of time. Table 7.44 lists how many actual detectors are successfully generated during the generation phase for each test. 
Table 7.44: Detectors generated based on testing matrix

\begin{tabular}{|l|c|c|}
\hline Damage pattern & $\begin{array}{c}\text { Transient damage } \\
\text { testing data }\end{array}$ & $\begin{array}{c}\text { Steady damage } \\
\text { testing data }\end{array}$ \\
\hline 1 (undamaged) & 1964 & 1931 \\
\hline 2 & 729 & 756 \\
\hline 3 & 680 & 499 \\
\hline 4 & 1612 & 1589 \\
\hline 5 & 1586 & 1492 \\
\hline 6 & 1551 & 1660 \\
\hline 7 & 1620 & 1631 \\
\hline 8 & 1707 & 1641 \\
\hline 9 & 1585 & 1599 \\
\hline
\end{tabular}

Once the AIS is trained, it is tested with the specific damage pattern of a damage state, represented by the data AIS_test contains. This is repeated for all damage patterns over all damage states. The results for the transient damage tests are listed Table 7.45 and summarized in Table 7.46. Likewise, the results for steady damage tests are listed in Table 7.47 and summarized in Table 7.48.

Table 7.45: Dual sensor AIS transient results

\begin{tabular}{|c|c|c|}
\hline Damage pattern & $\begin{array}{c}\text { \% Correct } \\
\text { (out of 100) }\end{array}$ & $\begin{array}{c}\text { \% Incorrect } \\
\text { (out of 100) }\end{array}$ \\
\hline 1 (undamaged) & 100.00 & 0.00 \\
\hline 2 & 99.5 & 0.5 \\
\hline 3 & 98 & 2 \\
\hline 4 & 100 & 0 \\
\hline 5 & 97 & 3 \\
\hline 6 & 99.5 & 0.5 \\
\hline 7 & 100 & 0 \\
\hline 8 & 100 & 0 \\
\hline 9 & 95 & 5 \\
\hline
\end{tabular}

Table 7.46: Dual Sensor AIS transient summary

\begin{tabular}{|c|c|c|}
\hline & $\begin{array}{c}\text { Classified } \\
\text { undamaged }\end{array}$ & $\begin{array}{c}\text { Classified } \\
\text { damaged }\end{array}$ \\
\hline $\begin{array}{c}\text { Actual } \\
\text { undamaged }\end{array}$ & $100 \%$ & $0 \%$ \\
\hline $\begin{array}{c}\text { Actual } \\
\text { damaged }\end{array}$ & $1.38 \%$ & $98.62 \%$ \\
\hline
\end{tabular}

Table 7.47: Dual sensor AIS steady damage results

\begin{tabular}{|c|c|c|}
\hline Damage pattern & $\begin{array}{c}\text { \% Correct } \\
\text { (out of 100) }\end{array}$ & $\begin{array}{c}\text { \% Incorrect } \\
\text { (out of 100) }\end{array}$ \\
\hline 1 (undamaged) & 100.00 & 0.00 \\
\hline 2 & 95 & 5 \\
\hline 3 & 96 & 4 \\
\hline 4 & 98.5 & 1.5 \\
\hline 5 & 95.5 & 4.5 \\
\hline 6 & 99.5 & 0.5 \\
\hline 7 & 99.5 & 0.5 \\
\hline 8 & 98.5 & 1.5 \\
\hline 9 & 97.5 & 2.5 \\
\hline
\end{tabular}

Table 7.48: Dual sensor AIS steady damage summary

\begin{tabular}{|c|c|c|}
\hline & $\begin{array}{c}\text { Classified } \\
\text { undamaged }\end{array}$ & $\begin{array}{c}\text { Classified } \\
\text { damaged }\end{array}$ \\
\hline $\begin{array}{c}\text { Actual } \\
\text { undamaged }\end{array}$ & $100 \%$ & $0 \%$ \\
\hline $\begin{array}{c}\text { Actual } \\
\text { damaged }\end{array}$ & $2.5 \%$ & $97.5 \%$ \\
\hline
\end{tabular}


To verify this method, the AIS is retested with data from sensors two and three. All other setup parameters are similar to the ones described above. In summary, the results using sensors two and three are similar to that of using one and two. For more detail, please see Appendix A.

\subsubsection{Summary}

\section{Approach One- Full Sensor Data}

Due to its nature, the AIS algorithm achieves a perfect 100\% undamaged detection rate. In addition, it was able to detect the most severe damage pattern (pattern \#3) with average accuracy of $\mathbf{7 4 . 2 5 \%}$. When operating in its current fashion where it is given inputs from all sensors, the NS falters in classifying the lesser damaged patterns. Examining the data for any damage pattern reveals a that the 4 dimension state space is mostly empty near one end, while the other end is intermingled with both undamaged and damaged points. For the AIS, that translates to a state space where certain areas can be covered with a few detectors with large radii, leaving the remainder which is divided into countless pockets of non-self space. These pockets must be filled in by detectors; however, it would require much more than 2,000 detectors. As a result, during testing, many damaged datasets slip through as undamaged, returning a high number of false positives. The AIS detected more damaged datasets when tested with transient damage compared to steady damage. This is due to the presence of the "spike" in approximation level 7 coefficients when the building became damaged. The spike affected the datasets, but not sufficiently for a significant outcome. Table 7.49 summarizes AIS's approach one results. 
Table 7.49: Approach one AIS result summary

\begin{tabular}{|l|c|c|}
\hline Damage type & Correct undamaged & Correct damaged \\
\hline Transient & $100 \%$ & $59.69 \%$ \\
\hline Steady & $100 \%$ & $50.19 \%$ \\
\hline Average & $\mathbf{1 0 0 \%}$ & $54.94 \%$ \\
\hline
\end{tabular}

\section{Approach Two- Dual Sensor Data}

The attempt to improve the AIS by reducing the state space it must cover ended in promising results. By considering data only from the first and fourth floor sensors, the AIS now has to cover a two dimensional state space instead of four. This is a much more achievable task: the variable overlapping radii NS algorithm accomplished this with an average of $\mathbf{1 , 4 4 8}$ detectors while testing transient damage data, and $\mathbf{1 , 4 2 2}$ detectors while testing steady damage data. See Appendix A for some visualized examples.

In this configuration, the AIS retains its perfect $100 \%$ undamaged state detection rate while vastly improving its damaged state detection accuracy. This is the direct result of the detectors being able to cover a majority of the state space. Again, the AIS performed better detecting transient damage patterns versus steady damage, but in this case, the discrepancy is much smaller. Table 7.50 summarizes AIS's approach two results.

Table 7.50: Approach two AIS result summary

\begin{tabular}{|l|c|c|}
\hline Damage type & Correct undamaged & Correct damaged \\
\hline Transient & $100 \%$ & $98.62 \%$ \\
\hline Steady & $100 \%$ & $97.5 \%$ \\
\hline Average & $\mathbf{1 0 0 \%}$ & $\mathbf{9 8 . 0 6 \%}$ \\
\hline
\end{tabular}




\section{CHAPTER 8 : CONCLUSION \& FURTHER RESEARCH}

The primary goal of this thesis is to evaluate and compare the potential use of artificial neural networks (ANNs) and negative selection (NS) algorithms as viable nondestructive evaluation (NDE) systems for structural health monitoring (SHM). The use of energy ratios based upon discrete wavelet transforms (DWT) is also tested as a potential means to filter and represent damage data.

The DWT was an effective tool for processing the raw acceleration data generated by Datagen. Using a 'db4' mother wavelet, level 7 approximation coefficients as well as energy ratios allowed processing power to be reduced exponentially while revealing promising detail for the ANN and NS algorithm to process upon.

The artificial neural network's success makes it a promising NDE method. The multi-damage neural network demonstrates a 100\%/94.43\% effectiveness in distinguishing between undamaged/damaged states. While it was unable to confidently distinguish between the lesser damage patterns, the network did identify the most severe damage case $78.39 \%$ of the time.

Since it relies upon experimental data rather than meticulously formulated mathematical analysis, the neural network's effectiveness can be crucially dependent upon the training data. This is demonstrated in the change in effectiveness whether the network is tested with native and non-native data. In every situation, the network performed better when tested with native data. The Boolean network's effectiveness suffered most when tested with non-native data. The multi-damage network setup, 
however, proved to be very robust in classifying native as well as non-native data. Table 8.1 summarizes the neural network’s overall average performance.

Table 8.1: Neural network average summary

\begin{tabular}{|l|c|c|}
\hline & Correct Undamaged & Correct Damaged \\
\hline Boolean network approach & $58.83 \%$ & $80.17 \%$ \\
\hline Multi-damage network approach & $100 \%$ & $92.47 \%$ \\
\hline
\end{tabular}

Despite its shortcomings, a neural network is still a formidable tool that should not be overlooked when developing SHM systems. With more effort spent upon optimizing the network, even more impressive results can be expected. Since good training data is paramount in creating an effective neural network, further research should be performed in improving that aspect, whether it is reshaping the training data to be more noise-resistant, or considering integrating feedback loops. Many other neural network parameters remain unexplored in this thesis, and await further development.

While the ANN trumps classical mathematical methods in terms of simplicity and ease in modeling nonlinear systems, the AIS negative selection algorithm takes it one step further. Requiring only “self” data for training, the NS algorithm removes ANN's requirement for training data over the entire detection spectrum. It is capable of distinguishing between undamaged/damaged states more accurately than the neural network, while requiring less diverse sources of data. The AIS also operated effectively with only two out of four sensor datasets.

Testing the AIS with either transient or steady damage has little distinction. Since the AIS does not require damage training data, there is no distinction between native and non-native testing damage data. Also, due to its nature, a properly set-up NS algorithm should rarely if ever return false positive results. This is confirmed in the simulations, 
where the NS algorithm returned $0 \%$ false positives in every damage pattern. Table 8.2 summarizes the AIS’s overall average performance.

Table 8.2: Neural network average summary

\begin{tabular}{|l|c|c|}
\hline & Correct Undamaged & Correct Damaged \\
\hline Full sensor approach & $100 \%$ & $54.94 \%$ \\
\hline Dual sensor approach & $100 \%$ & $98.06 \%$ \\
\hline
\end{tabular}

Detector coverage directly affects the effectiveness of a NS algorithm. While this thesis uses variable radius overlapping detectors to improve coverage, further work should be invested in improving how detectors are generated. Currently, each detector is randomly generated across the entire state space. Varying the state space dynamically to exclude areas already covered by large detectors can speed up the detector generation phase. Also with further research, perhaps the severity and the spatial location of the damage can also be determined. All of these uncharted opportunities make the NS algorithm a remarkable choice that merits further research for use in SHM systems. 


\section{REFERENCES}

[1] H. Sohn, C. R. Farrar, F. Hemez and J. Czarnecki, "A Review of Structural Health Monitoring Literature 1996-2001," Los Alamos National Laboratory, New Mexico, 2002.

[2] C. R. Farrar, S. W. Doebling and D. A. Nix, "Vibration-based Structural Damage Identification," Experimental Modal Analysis, vol. 359, no. 1778, pp. 131-149, 2001.

[3] A. Bulut, A. K. Singh, P. Shin, T. Fountain and H. Jasso, "Real-time Nondestructive Structural Health Monitoring Using Support Vector Machines and Wavelets," Department of Computer Science, UC Santa Barbara, Santa Barbara.

[4] D. Paterson, "Structural Health Monitoring of America's Infrastructure," STRUCTURE, p. 37, May 2014.

[5] G. Fu and A. Moosa, "Structural Damage Diagnosis using High Resolution Images," Structural Safety, vol. 23, no. 4, pp. 281-295, 2001.

[6] S. Saadat, M. N. Noori, G. D. Buckner, T. Furukawa and Y. Suzuki, "Structural health monitoring and damage detection using an intelligent parameter varying (IPV) technique," Elsevier Ltd, 2004.

[7] J. Thomas, D. Kosnik, M. Kotowsky, D. Marron and J. Schofer, "Structural Health Monitoring of Civil Structures," Northwestern University, Evanston, 2008.

[8] A. Belisario-Briceno, S. Zedek, T. Camps, R. Francois and C. Escriba, " SHM Based on Modal Analysis: Accelerometer and Piezoelectric Transducers Instrumentation for Civil Engineering in Heterogeneous Structures," in EWSHM - 7th European Workshop on Structural Health Monitoring, Nantes, 2014.

[9] G. Hearn and R. Testa, "Modal Analysis for Damage Detection in Structures," Journal of Structural Engineering, vol. 117, no. 10, pp. 3042-3063, 1991.

[10] C. S. Huang, S. L. Hung, C. I. Lin and W. C. Su, "A Wavelet-Based Approach to Identifying Structural Modal Parameters from Seismic Response and Free Vibration Data," Computer-Aided Civil and Infrastructure Engineering, no. 20, pp. 408-423, 2005.

[11] M. A. Lotfollahi-Yaghin and M. A. Hesari, "Using Wavelet Analysis in Crack Detection at the Arch Concrete Dam," World Applied Sciences Journal, pp. 691-704, 2008. 
[12] Y. Mizuno, E. Monroig and Y. Fujino, "Wavelet Decomposition-Based Approach for Fast Damage Detection of Civil Structures," Journal of Infrastructure Systems, vol. 14, no. 1, pp. 27-32, 2008.

[13] J. Morlier, M. Bergh and L. Mevel, "Modeshapes recognition using Fourier descriptors: a simple SHM example.," in IMAC XXX Conference and exposition on structural dynamics, Jacksonville, 2011.

[14] Z. Hou, M. Noori and R. S. Amand, "Wavelet Based Approach for Structural Damage Detection," Journal of Engineering Mechanics, vol. 126, no. 7, 2000.

[15] C.-C. Chang and L.-W. Chen, "Vibration damage detection of a Timoshenko Beam by Spatial Wavelet Based Approach," Applied Acoustics, no. 64, pp. 1217-1240, 2003.

[16] Y. Wu and R. Du, "Feature Extraction and Assessment Using Wavelet Packets for Monitoring of Machining Processes," Mechanical Systems and Signal Processing , vol. 10, no. 1, pp. 29-53, 1996.

[17] C. Kai and Y. Hamamatsu, "An Adaptive Hybrid Wavelet Neural Network and its Application," in IEEE International Conference on Robotics and Biomimetics, Shenyang, 2004.

[18] A. Hera and Z. Hou, "Application of Wavelet Approach for ASCE Structural Health," Journal of Engineering Mechanics, pp. 96-104, 2004.

[19] C. Kao and S.-L. Hung, "Detection of Structural Damage via Free Vibration Responses Generated by AApproximating Artificial Neural Networks," Computers \& Structures, no. 81, pp. 2631-2644, 2003.

[20] P. Tsou and M. H. H. Shen, "Structural Damage Detection and Identification using Neural Networks," AIAA Journal, vol. 32, no. 1, pp. 176-183, 1997.

[21] D. A. Sofge, "Structural Health Monitoring Using Neural Network Based Vibration System Identification," in ANZIIS '94 - Australian New Zealand Intelligent Information Systems Conference, Brisbane, 1994.

[22] B. Chen and C. Zang, "Artificial Immune Pattern Recognition for Structure Damage Classification," Computers and Structures, no. 87, pp. 1394-1407, 2009.

[23] M. M. R. Taha, A. Noureldin, J. L. Lucero and T. J. Baca, "Wavelet Transform for Structural Health Monitoring: A Compendium of Uses and Features," Structural Health Monitoring, vol. 5, no. 3, pp. 267-329, 2006.

[24] MATLAB, "Wavelet L Vector," MATLAB, 2004. 
[25] X. G. J. a. G. J. H. Wu, "Use of Neural Networks in Detection of Structural Damage," Computers and Structures, vol. 42, no. 4, pp. 649-659, 1992.

[26] MATLAB, "Levenberg-Marquardt (Trainlm)," 2004.

[27] M. Middlemiss, "Positive and Negative Selection in a Multilayer Artificial Immune System," Department of Information Science, Dunedin, 2006.

[28] L. De Castro and F. Von Zuben, "Artificial Immune Systems: Part I - Basic Theory and Applications," Technical Report, 1999.

[29] Z. Ji and D. Dasgupta, "Applicability Issues of the Real-Valued Negative Selection Algorithms," in Genetic and Evolutionary Computation Conference (GECCO), Seattle, 2006.

[30] E. Johnson, H. Lam, L. Katafygiotis and J. Beck, "Phase 1 IASC-ASCE Structural Health Monitoring Benchmark Problem Using Simulated Data," Journal of Engineering Mechanics, vol. 130, no. 1, pp. 3-15, 2004.

[31] Z. Hou and A. Hera, "Progress of Phase II Study of the ASCE Health Monitoring," in 15th ASCE Engineering Mechanics Conference, New York, 2002.

[32] H. Kim and H. Melhem, "Damage Detection of Structures by Wavelet Analysis," Engineering Structures, no. 26, pp. 347-362, 2004.

[33] Y. Zhou, S. Tang, C. Zang and R. Zhou, "An Artificial Immune Pattern Recognition Approach for Damage Classifications in Structures," in Advances in Information Technology and Industry Applications, Berlin, Springer-Verlag, 2012, pp. 11-77. 


\section{APPENDICES}

\section{Appendix A: Additional Tables and Figures}

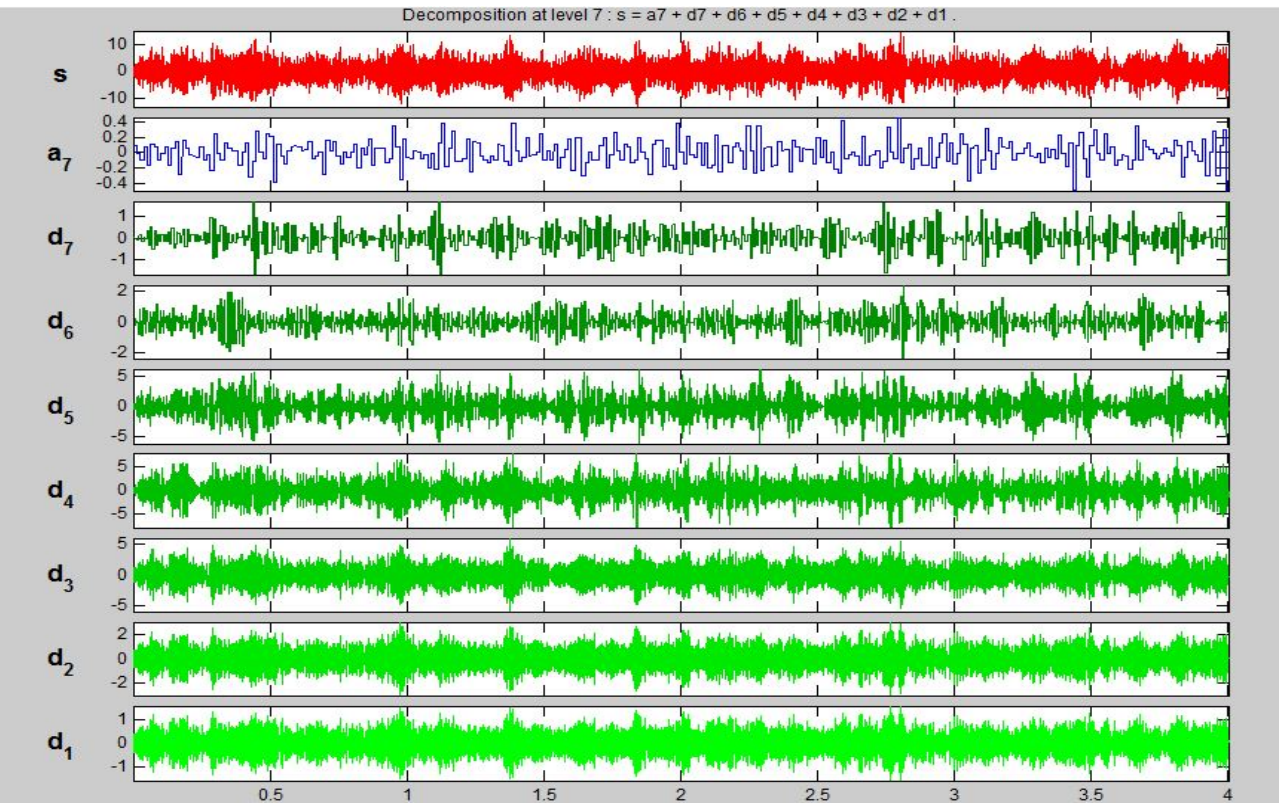

Figure A.1: DWT using 'Haar’ wavelet on sensor \#2, damage pattern \#7

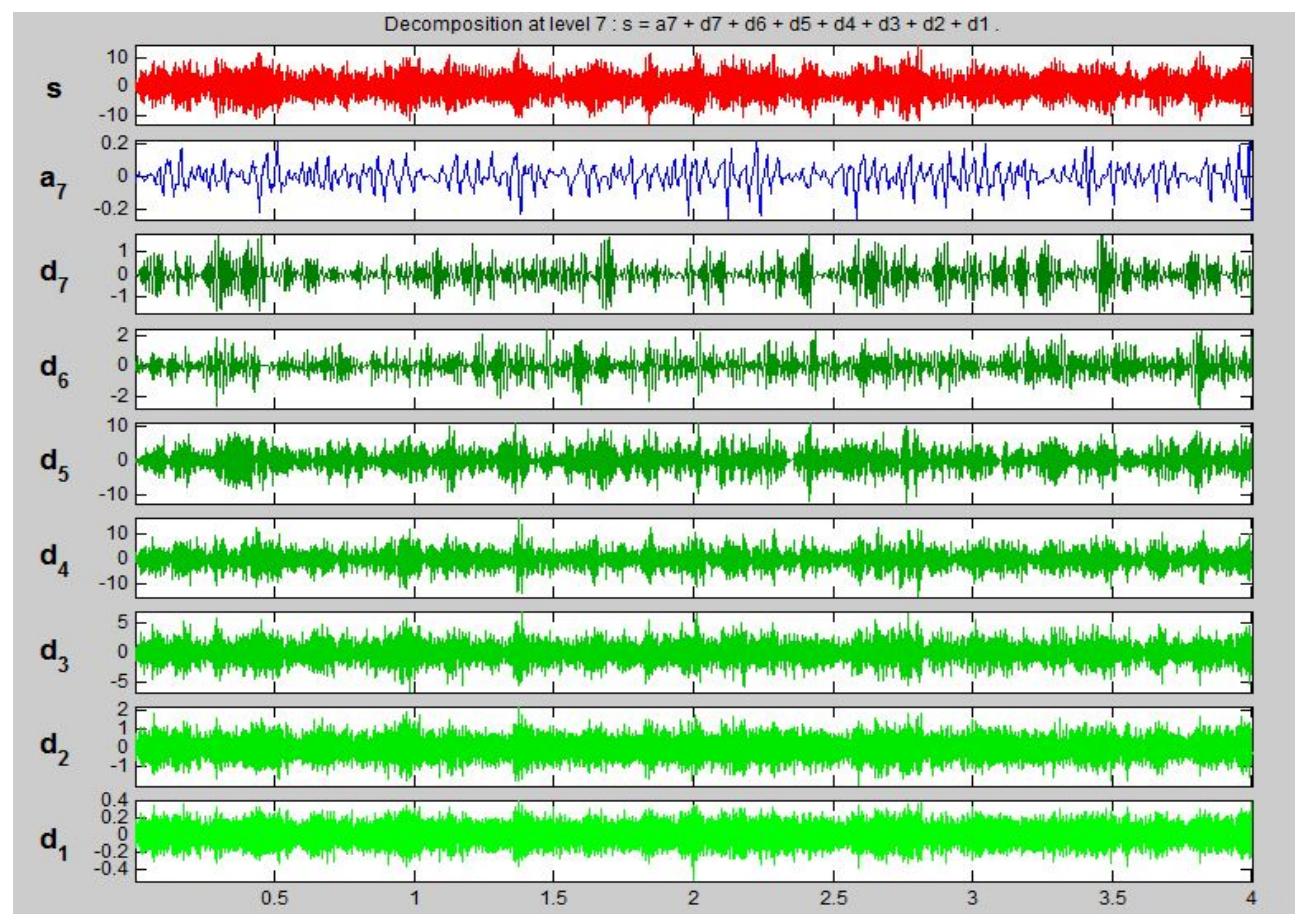

Figure A.2: DWT using 'db2’ wavelet on sensor \#2, damage pattern \#7 


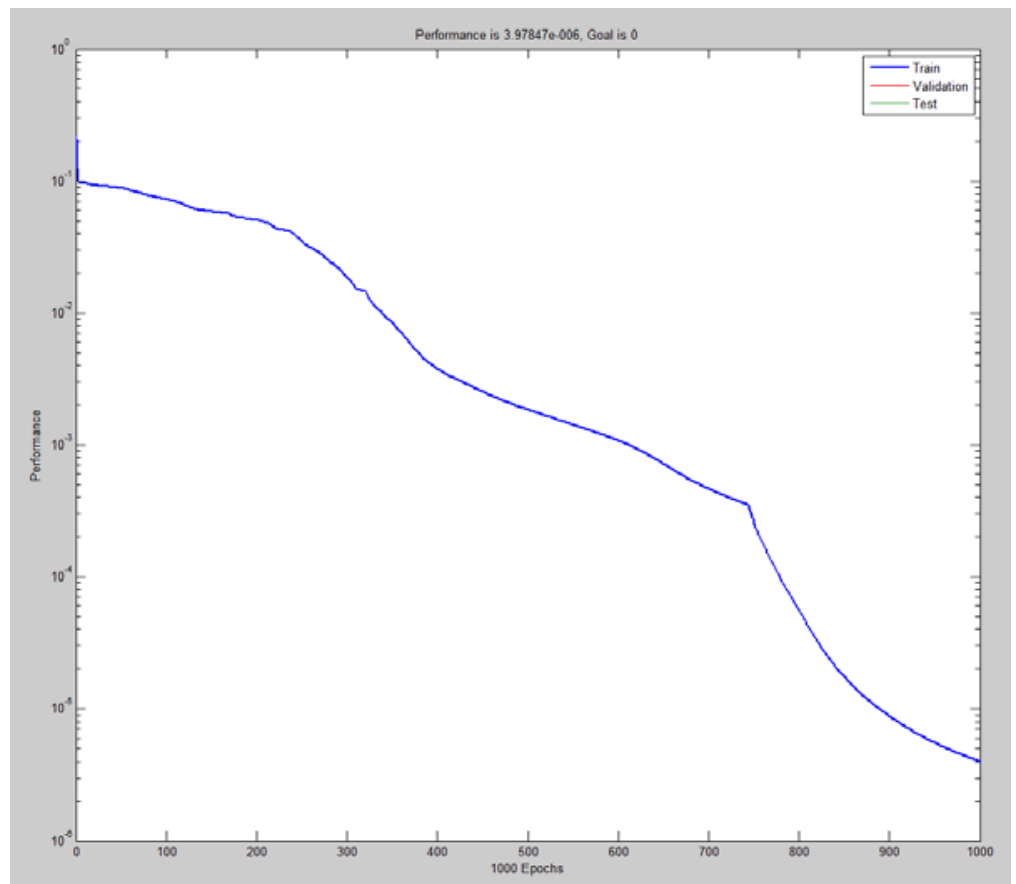

Figure A.3: Neural network net_b1's training MSE curve

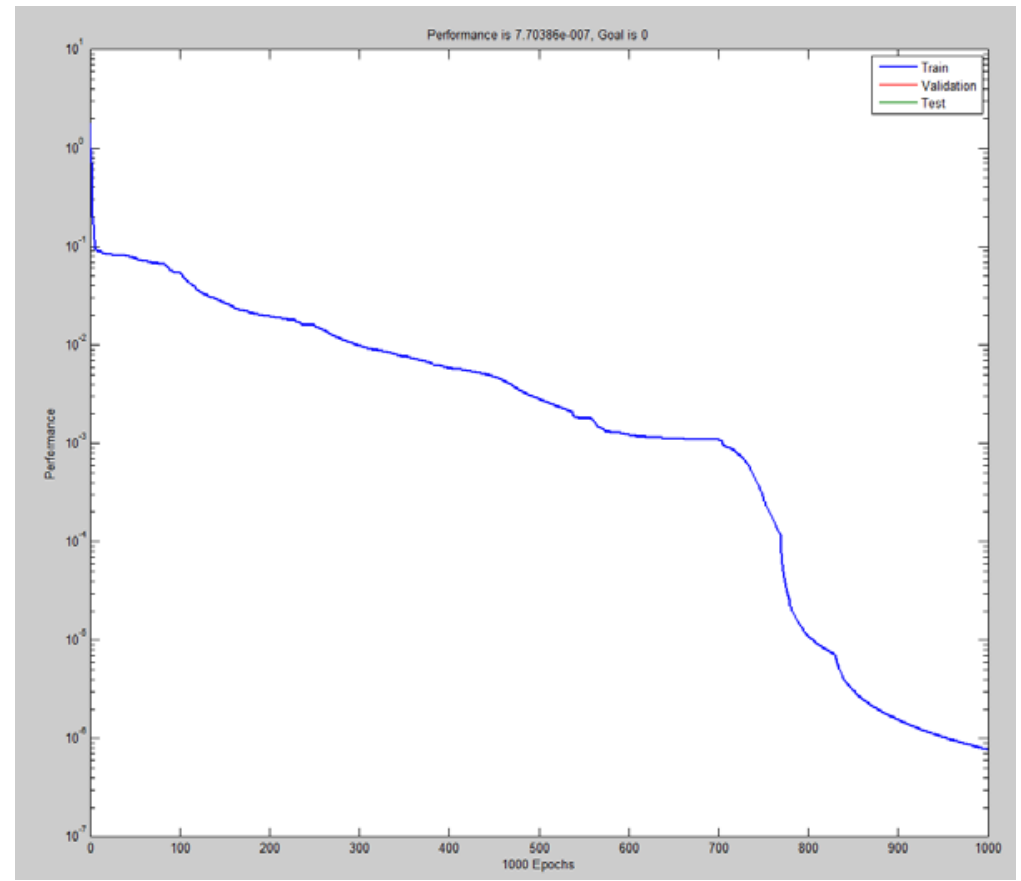

Figure A.4: Neural network net_b2's training MSE curve 


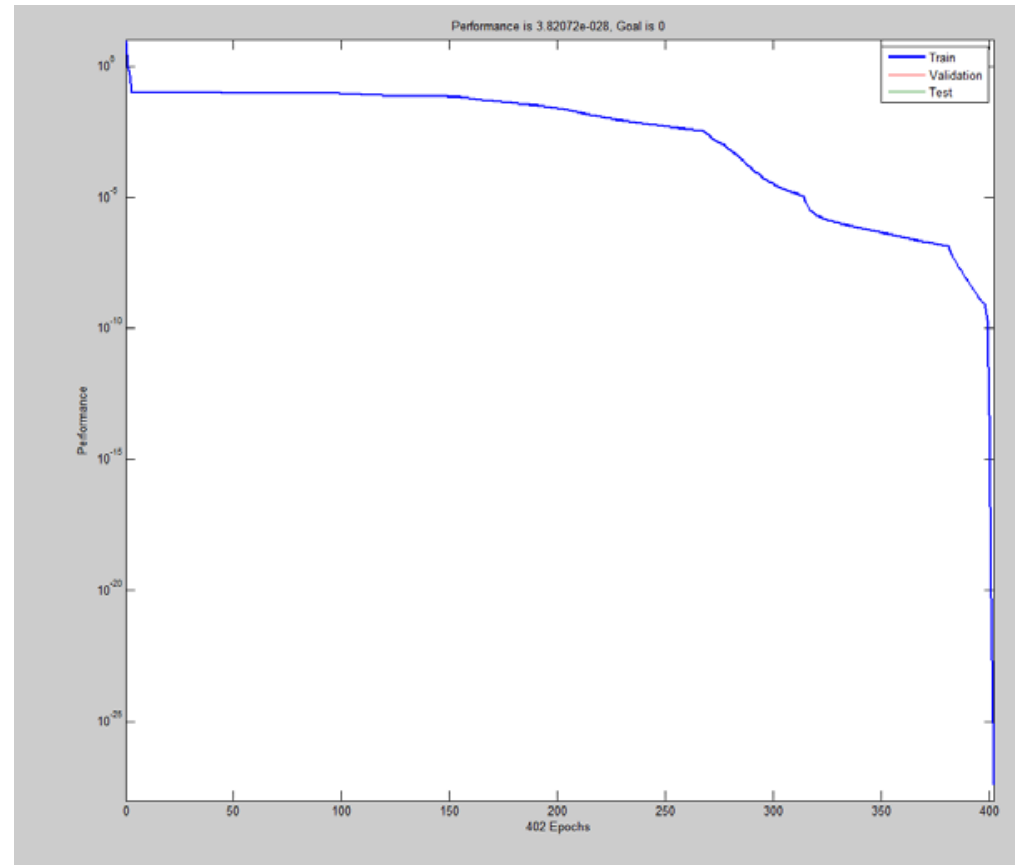

Figure A.5: Neural network net_b1a's training MSE curve 


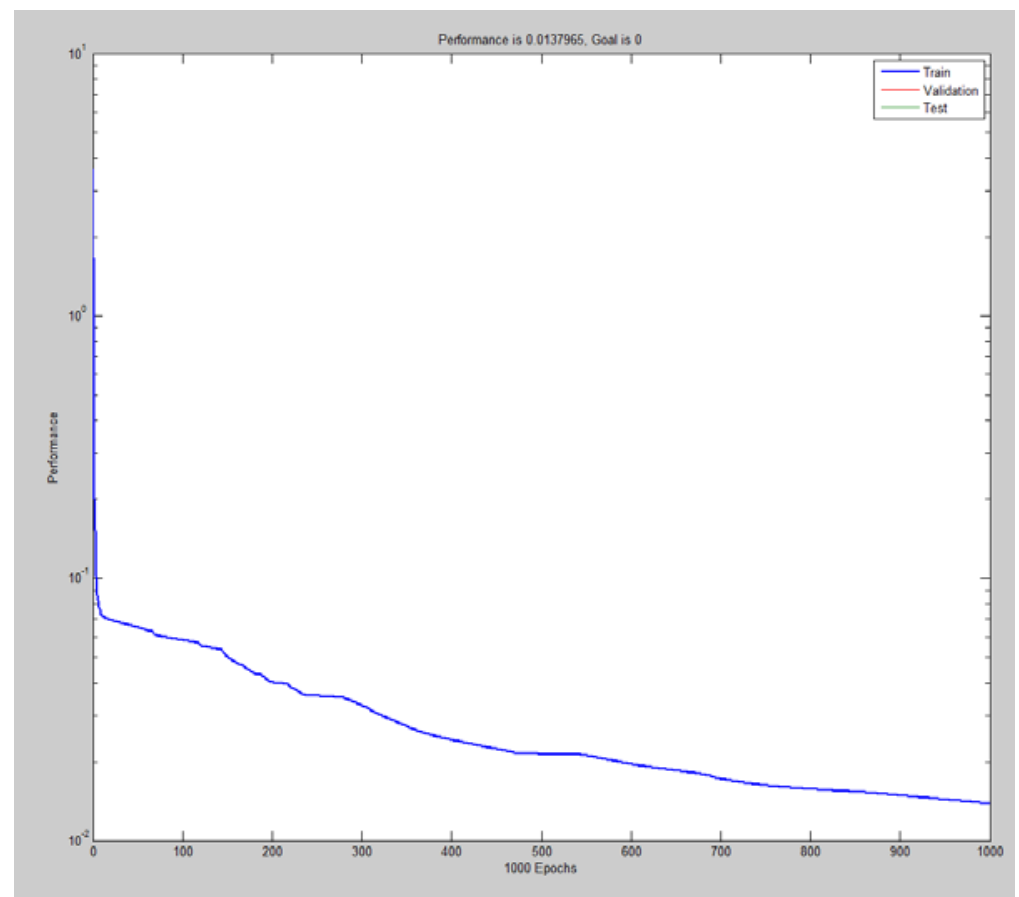

Figure A.6: Neural network net_1shot_1's training MSE curve

Table A.1: Neural network net_1shot_1's transient damage confusion matrix

\begin{tabular}{|c|c|c|c|c|c|c|c|c|c|}
\begin{tabular}{|c|c|c|c|c|c|c|c|} 
Actuar Detecting. \\
Dmg.
\end{tabular} & $\mathbf{1}$ & $\mathbf{2}$ & $\mathbf{3}$ & $\mathbf{4}$ & $\mathbf{5}$ & $\mathbf{6}$ & $\mathbf{7}$ & $\mathbf{8}$ & $\mathbf{9}$ \\
\hline $\mathbf{1}$ & 100.00 & 0.00 & 0.00 & 0.00 & 0.00 & 0.00 & 0.00 & 0.00 & 0.00 \\
\hline $\mathbf{2}$ & 4.93 & 16.14 & 15.70 & 13.45 & 11.21 & 11.21 & 9.42 & 8.52 & 9.42 \\
\hline $\mathbf{3}$ & 6.15 & 27.86 & 31.43 & 8.57 & 5.71 & 5.71 & 0.71 & 8.57 & 11.43 \\
\hline $\mathbf{4}$ & 12.09 & 10.16 & 13.46 & 10.16 & 9.07 & 8.52 & 12.09 & 12.91 & 11.54 \\
\hline $\mathbf{5}$ & 8.36 & 9.60 & 15.17 & 11.76 & 11.76 & 11.76 & 7.74 & 11.15 & 12.69 \\
\hline $\mathbf{6}$ & 7.87 & 14.58 & 15.16 & 11.37 & 11.37 & 11.95 & 7.87 & 9.04 & 10.79 \\
\hline $\mathbf{7}$ & 12.59 & 18.53 & 13.99 & 7.69 & 9.44 & 6.64 & 10.14 & 10.84 & 10.14 \\
\hline $\mathbf{8}$ & 9.70 & 14.85 & 13.64 & 10.30 & 8.48 & 9.70 & 10.30 & 12.12 & 10.91 \\
\hline $\mathbf{9}$ & 9.27 & 12.78 & 12.14 & 10.54 & 9.90 & 12.46 & 10.54 & 9.27 & 13.10 \\
\hline
\end{tabular}

Table A.2: Neural network net_1shot_1's steady damage confusion matrix

\begin{tabular}{|c|c|c|c|c|c|c|c|c|c|}
\hline $\begin{array}{l}\text { Detected } \\
\text { Actual } \\
\text { Dmg. }\end{array}$ & 1 & 2 & 3 & 4 & 5 & 6 & 7 & 8 & 9 \\
\hline 1 & 100.00 & 0.00 & 0.00 & 0.00 & 0.00 & 0.00 & 0.00 & 0.00 & 0.00 \\
\hline 2 & 5.13 & 64.10 & 12.82 & 3.42 & 3.42 & 3.42 & 5.13 & 0.85 & 1.71 \\
\hline 3 & 4.08 & 9.18 & 78.57 & 1.02 & 0.00 & $\begin{array}{l}1.02 \\
\end{array}$ & 2.04 & 4.08 & 0.00 \\
\hline 4 & 3.14 & 8.81 & 9.43 & 46.54 & 4.40 & 6.92 & 8.18 & 6.29 & 6.29 \\
\hline 5 & 6.31 & 7.21 & 9.01 & 5.41 & 41.44 & 9.91 & 12.61 & 7.21 & 0.90 \\
\hline 6 & 9.58 & 7.78 & 7.19 & 7.19 & 10.18 & 46.11 & 3.59 & 5.99 & 2.40 \\
\hline 7 & 6.62 & 9.27 & 6.62 & 3.31 & 3.97 & 5.30 & 49.01 & 9.27 & 6.62 \\
\hline 8 & 7.10 & 8.39 & 10.32 & 3.87 & 4.52 & 3.87 & 6.45 & 49.03 & 6.45 \\
\hline 9 & 6.08 & 5.41 & 8.78 & 6.76 & 5.41 & 4.73 & 6.76 & 5.41 & 50.68 \\
\hline
\end{tabular}




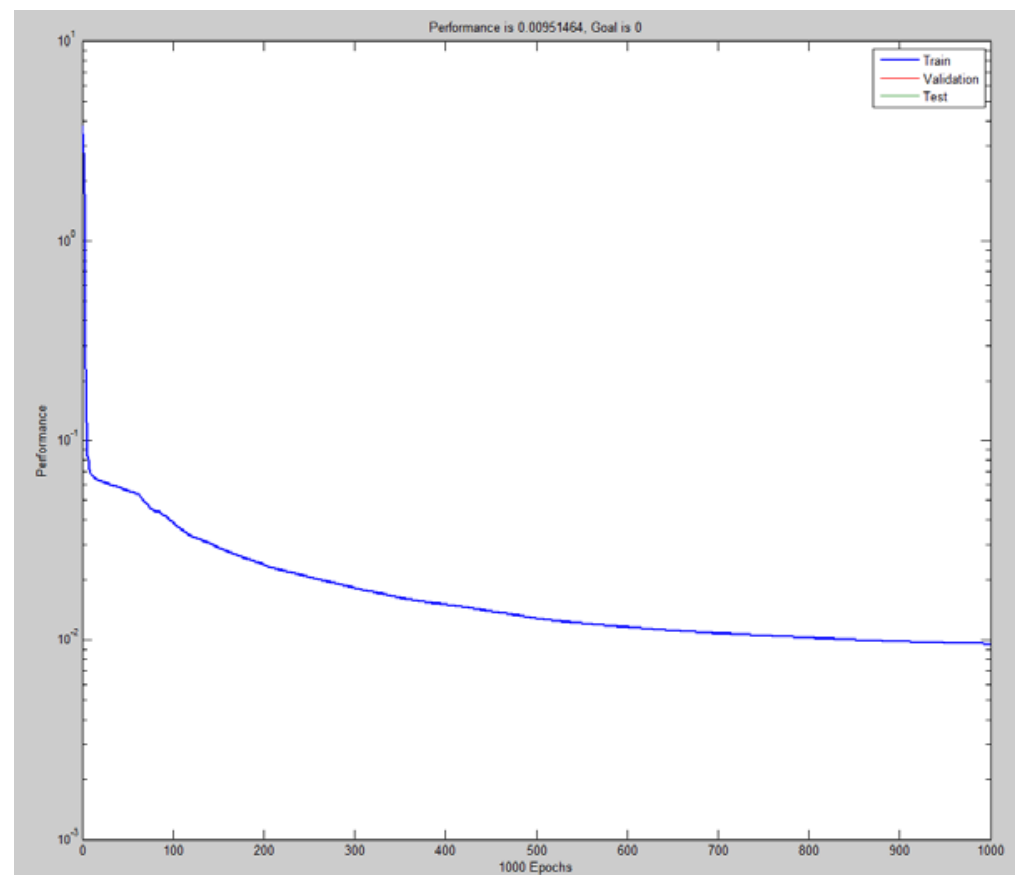

Figure A.7: Neural network net_1shot_2's training MSE curve

Table A.3: Neural network net_1shot_2's transient damage confusion matrix

\begin{tabular}{|c|c|c|c|c|c|c|c|c|c|}
\begin{tabular}{|c|c|c|c|c|c|c|c|} 
Actua- Detecting. \\
Dmg.
\end{tabular} & $\mathbf{1}$ & $\mathbf{2}$ & $\mathbf{3}$ & $\mathbf{4}$ & $\mathbf{5}$ & $\mathbf{6}$ & $\mathbf{7}$ & $\mathbf{8}$ & $\mathbf{9}$ \\
\hline $\mathbf{1}$ & 100.00 & 0.00 & 0.00 & 0.00 & 0.00 & 0.00 & 0.00 & 0.00 & 0.00 \\
\hline $\mathbf{2}$ & 1.37 & 58.90 & 10.96 & 6.16 & 8.22 & 4.11 & 2.74 & 2.74 & 4.79 \\
\hline $\mathbf{3}$ & 0.00 & 12.63 & 75.79 & 2.11 & 1.05 & 2.11 & 0.00 & 3.16 & 3.16 \\
\hline $\mathbf{4}$ & 7.73 & 6.76 & 9.18 & 41.06 & 6.76 & 6.28 & 6.76 & 8.70 & 6.76 \\
\hline $\mathbf{5}$ & 4.94 & 7.41 & 5.56 & 5.56 & 45.68 & 10.49 & 5.56 & 4.32 & 10.49 \\
\hline $\mathbf{6}$ & 4.35 & 10.14 & 12.32 & 3.62 & 5.07 & 43.48 & 6.52 & 6.52 & 7.97 \\
\hline $\mathbf{7}$ & 7.14 & 5.95 & 5.95 & 7.14 & 7.74 & 8.93 & 49.40 & 4.76 & 2.98 \\
\hline $\mathbf{8}$ & 3.01 & 9.64 & 6.63 & 6.63 & 6.02 & 4.82 & 4.22 & 51.20 & 7.83 \\
\hline $\mathbf{9}$ & 5.26 & 9.77 & 9.02 & 5.26 & 3.01 & 1.50 & 4.51 & 5.26 & 56.39 \\
\hline
\end{tabular}

Table A.4: Neural network net_1shot_2's steady damage confusion matrix

\begin{tabular}{|c|c|c|c|c|c|c|c|c|c|}
\hline $\begin{array}{l}\text { Detected } \\
\text { Actua } \\
\text { Dmg. }\end{array}$ & 1 & 2 & 3 & 4 & 5 & 6 & 7 & 8 & 9 \\
\hline 1 & 100.00 & 0.00 & 0.00 & 0.00 & 0.00 & 0.00 & 0.00 & 0.00 & 0.00 \\
\hline 2 & 8.57 & 18.37 & 14.69 & 11.84 & 9.80 & 11.02 & 8.57 & 11.43 & 5.71 \\
\hline 3 & 3.67 & 25.69 & 36.70 & 5.50 & 3.67 & 3.67 & 10.09 & 8.26 & 2.75 \\
\hline 4 & 13.77 & 12.19 & 8.80 & 11.74 & 10.61 & 9.71 & 8.58 & 12.19 & 12.42 \\
\hline 5 & 8.10 & 13.38 & 14.44 & 9.51 & 10.92 & 9.15 & 10.21 & 13.73 & 10.56 \\
\hline 6 & 8.42 & 12.46 & 19.19 & 7.07 & 11.78 & 10.77 & 11.45 & 12.12 & 6.73 \\
\hline 7 & 7.87 & 7.87 & $\begin{array}{l}13.76 \\
\end{array}$ & 8.15 & 10.96 & 11.24 & 15.45 & 11.52 & 13.20 \\
\hline 8 & 14.47 & $\begin{array}{l}14.78 \\
\end{array}$ & 9.12 & 10.06 & 9.12 & 10.38 & 9.75 & 13.21 & 9.12 \\
\hline 9 & 9.54 & 11.84 & 18.75 & 11.84 & 10.86 & 9.21 & 7.24 & 10.53 & 10.20 \\
\hline
\end{tabular}




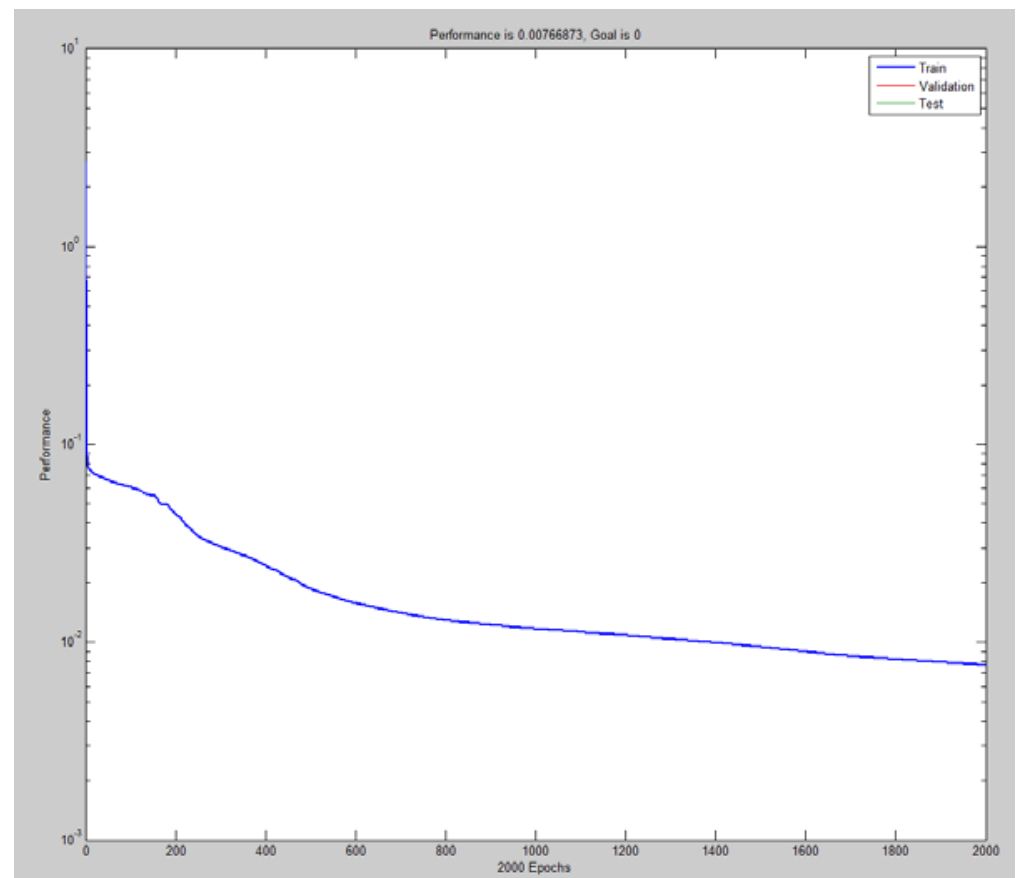

Figure A.8: Neural network net_1shot_1a's training MSE curve

Table A.5: Neural network net_1shot_1a's transient damage confusion matrix

\begin{tabular}{|c|c|c|c|c|c|c|c|c|c|}
\hline \multirow{2}{*}{$\begin{array}{l}\text { Detected } \\
\text { Actuaal Dmg. } \\
\text { Dmg. }\end{array}$} & 1 & 2 & 3 & 4 & 5 & 6 & 7 & 8 & 9 \\
\hline & 100.00 & 0.00 & 0.00 & 0.00 & 0.00 & 0.00 & 0.00 & 0.00 & 0.00 \\
\hline 2 & 4.04 & 17.68 & 16.16 & 10.61 & 12.12 & 13.13 & 6.06 & 9.60 & 10.61 \\
\hline 3 & 2.14 & 27.14 & 32.86 & 10.71 & 6.43 & 5.00 & 5.71 & 6.43 & 3.57 \\
\hline 4 & 11.85 & 11.85 & 8.67 & 12.43 & 11.56 & 10.98 & 13.01 & 9.54 & 10.12 \\
\hline 5 & 9.26 & 11.16 & 11.64 & 10.93 & 12.35 & 11.64 & 9.03 & 12.11 & 11.88 \\
\hline 6 & 11.85 & 14.05 & 13.50 & 10.47 & 7.99 & 9.64 & 11.57 & 10.47 & 10.47 \\
\hline 7 & 10.24 & 13.25 & 13.55 & 10.24 & 9.04 & 9.04 & 9.94 & 10.84 & 13.86 \\
\hline 8 & 7.06 & 13.56 & 9.89 & 12.15 & 12.71 & 12.71 & 7.34 & 14.97 & 9.60 \\
\hline 9 & 12.95 & 12.12 & 17.08 & 10.19 & 8.54 & 9.37 & 11.57 & 9.37 & 8.82 \\
\hline
\end{tabular}

Table A.6: Neural network net_1shot_1a's steady damage confusion matrix

\begin{tabular}{|c|c|c|c|c|c|c|c|c|c|}
\hline \multirow{2}{*}{$\begin{array}{c}\text { Actual } \\
\text { Dmg. Dmg. }\end{array}$} & $\mathbf{1}$ & $\mathbf{2}$ & $\mathbf{3}$ & $\mathbf{4}$ & $\mathbf{5}$ & $\mathbf{6}$ & $\mathbf{7}$ & $\mathbf{8}$ & $\mathbf{9}$ \\
\hline & 100.00 & 0.00 & 0.00 & 0.00 & 0.00 & 0.00 & 0.00 & 0.00 & 0.00 \\
\hline $\mathbf{2}$ & 6.52 & 70.65 & 3.26 & 3.26 & 4.35 & 4.35 & 2.17 & 2.17 & 3.26 \\
\hline $\mathbf{3}$ & 3.03 & 9.09 & 80.81 & 2.02 & 1.01 & 0.00 & 2.02 & 2.02 & 0.00 \\
\hline $\mathbf{4}$ & 5.38 & 12.37 & 5.38 & 40.86 & 10.75 & 6.99 & 7.53 & 3.23 & 7.53 \\
\hline $\mathbf{5}$ & 7.43 & 2.86 & 6.86 & 8.00 & 44.00 & 7.43 & 5.71 & 9.14 & 8.57 \\
\hline $\mathbf{6}$ & 6.25 & 10.80 & 10.23 & 5.11 & 10.23 & 46.02 & 3.41 & 3.98 & 3.98 \\
\hline $\mathbf{7}$ & 2.99 & 5.39 & 6.59 & 7.78 & 8.98 & 6.59 & 46.71 & 9.58 & 5.39 \\
\hline $\mathbf{8}$ & 5.56 & 8.02 & 8.02 & 6.79 & 4.32 & 4.94 & 6.79 & 48.77 & 6.79 \\
\hline $\mathbf{9}$ & 9.19 & 7.57 & 9.73 & 5.95 & 7.57 & 5.41 & 6.49 & 4.32 & 43.78 \\
\hline
\end{tabular}


Table A.7 below lists the outcomes of the AIS parameter survey. The survey is accomplished in four steps, where all parameters are locked and one is changed in an attempt to find an optimal value. The numbers in the damage pattern represent the percentage correctly classified for each individual damage pattern.

Table A.7: AIS setup parameter survey

\begin{tabular}{|c|c|c|c|c|c|c|c|c|c|c|c|c|}
\hline & & & & & & ama & e pa & tern & cor & ectly & cla & ified (\%) \\
\hline N-norm & $\begin{array}{l}\text { Dets to } \\
\text { gen. }\end{array}$ & $\begin{array}{c}\text { Min det. } \\
\text { radius }\end{array}$ & $\begin{array}{l}\text { Radius } \\
\text { Overlap }\end{array}$ & 2 & 3 & 4 & 5 & 6 & 7 & 8 & 9 & $\begin{array}{l}\text { Avg. Accuracy } \\
(\%)\end{array}$ \\
\hline & & & Survey & num & er 0 & dete & tors & & & & & \\
\hline 4 & 100 & 0.001 & $50 \%$ & 36 & 62 & 7 & 6 & 10 & 0 & 7 & 0 & 16 \\
\hline & 250 & & & 41 & 67 & 9 & 8 & 10 & 1 & 7 & 0 & 17.875 \\
\hline & 691 & & & 60 & 78 & 13 & 12 & 15 & 8 & 12 & 6 & 25.5 \\
\hline & & & Survey 2 & orm & inste & $\mathrm{d}$ of & 4-no & & & & & \\
\hline 2 & 100 & 0.001 & $50 \%$ & 25 & 48 & 1 & 1 & 0 & 0 & 0 & 0 & 9.375 \\
\hline & 250 & & & 34 & 52 & 4 & 6 & 7 & 1 & 7 & 0 & 13.875 \\
\hline & 500 & & & 40 & 61 & 7 & 8 & 10 & 2 & 8 & 1 & 17.125 \\
\hline & 750 & & & 41 & 64 & 7 & 8 & 10 & 2 & 8 & 2 & 17.75 \\
\hline & 2000 & & & 55 & 71 & 14 & 15 & 16 & 14 & 14 & 8 & 25.875 \\
\hline & & & vey minin & Im d & etect & $\mathrm{r} \mathrm{rad}$ & us a & owe & & & & \\
\hline 2 & 2000 & 0.0001 & $50 \%$ & 55 & 73 & 14 & 15 & 16 & 13 & 14 & 6 & 25.75 \\
\hline & & 0.0005 & & 55 & 73 & 14 & 15 & 16 & 14 & 14 & 8 & 26.125 \\
\hline & & 0.001 & & 55 & 71 & 14 & 15 & 16 & 14 & 14 & 8 & 25.875 \\
\hline & & 0.005 & & 41 & 65 & 5 & 5 & 9 & 2 & 8 & 2 & 17.125 \\
\hline & & 0.01 & & 39 & 61 & 5 & 5 & 6 & 2 & 8 & 1 & 15.875 \\
\hline & & & Survey d & tecto & ove & lap c & low & & & & & \\
\hline 2 & 2000 & 0.0005 & $0 \%$ & 25 & 48 & 1 & 1 & 0 & 0 & 0 & 0 & 9.375 \\
\hline & & & $50 \%$ & 55 & 73 & 14 & 15 & 16 & 14 & 14 & 8 & 26.125 \\
\hline & & & $75 \%$ & 40 & 68 & 10 & 6 & 15 & 2 & 9 & 2 & 19 \\
\hline & & & $99 \%$ & 35 & 59 & 6 & 5 & 3 & 0 & 4 & 0 & 14 \\
\hline
\end{tabular}


Table A.8: Dual sensor (2\&3) AIS transient damage results

\begin{tabular}{|c|c|c|}
\hline Damage pattern & $\begin{array}{c}\text { \% Correct } \\
\text { (out of 100) }\end{array}$ & $\begin{array}{c}\text { \% Incorrect } \\
\text { (out of 100) }\end{array}$ \\
\hline 1 (undamaged) & 100 & 0 \\
\hline 2 & 92 & 8 \\
\hline 3 & 98.5 & 1.5 \\
\hline 4 & 99 & 1 \\
\hline 5 & 98.5 & 1.5 \\
\hline 6 & 100 & 0 \\
\hline 7 & 95 & 5 \\
\hline 8 & 100 & 0 \\
\hline 9 & 100 & 0 \\
\hline
\end{tabular}

Table A.9: Dual Sensor (2\&3) AIS transient damage summary

\begin{tabular}{|c|c|c|}
\hline & $\begin{array}{c}\text { Classified } \\
\text { undamaged }\end{array}$ & $\begin{array}{c}\text { Classified } \\
\text { damaged }\end{array}$ \\
\hline $\begin{array}{c}\text { Actual } \\
\text { undamaged }\end{array}$ & $100 \%$ & $0 \%$ \\
\hline $\begin{array}{c}\text { Actual } \\
\text { damaged }\end{array}$ & $2.12 \%$ & $97.88 \%$ \\
\hline
\end{tabular}

Table A.10: Dual sensor (2\&3) AIS steady damage results

\begin{tabular}{|c|c|c|}
\hline Damage pattern & $\begin{array}{c}\text { \% Correct } \\
\text { (out of 100) }\end{array}$ & $\begin{array}{c}\text { \% Incorrect } \\
\text { (out of 100) }\end{array}$ \\
\hline 1 (undamaged) & 100 & 0 \\
\hline 2 & 96 & 4 \\
\hline 3 & 90 & 10 \\
\hline 4 & 100 & 0 \\
\hline 5 & 97.5 & 2.5 \\
\hline 6 & 99.5 & 0.5 \\
\hline 7 & 100 & 0 \\
\hline 8 & 95 & 5 \\
\hline 9 & 99 & 1 \\
\hline
\end{tabular}

Table A.11: Dual Sensor (2\&3) AIS steady damage summary

\begin{tabular}{|c|c|c|}
\hline & $\begin{array}{c}\text { Classified } \\
\text { undamaged }\end{array}$ & $\begin{array}{c}\text { Classified } \\
\text { damaged }\end{array}$ \\
\hline $\begin{array}{c}\text { Actual } \\
\text { undamaged }\end{array}$ & $100 \%$ & $0 \%$ \\
\hline $\begin{array}{c}\text { Actual } \\
\text { damaged }\end{array}$ & $2.87 \%$ & $97.13 \%$ \\
\hline
\end{tabular}


Figure A.7 and Figure A.8 below visualize the AIS dual-sensor approach's detection results. Figure A.7 shows the results for testing the AIS with steady damage data from damage pattern 2. Figure A.8 shows the results for testing the AIS with steady damage data from damage pattern 7. For each pair, the following legend applies:

- Black dots denote undamaged training datasets

- Green dots denote undamaged testing datasets

- Red dots denote damaged testing datasets

- Blue circles outline each generated detector's non-self cover space
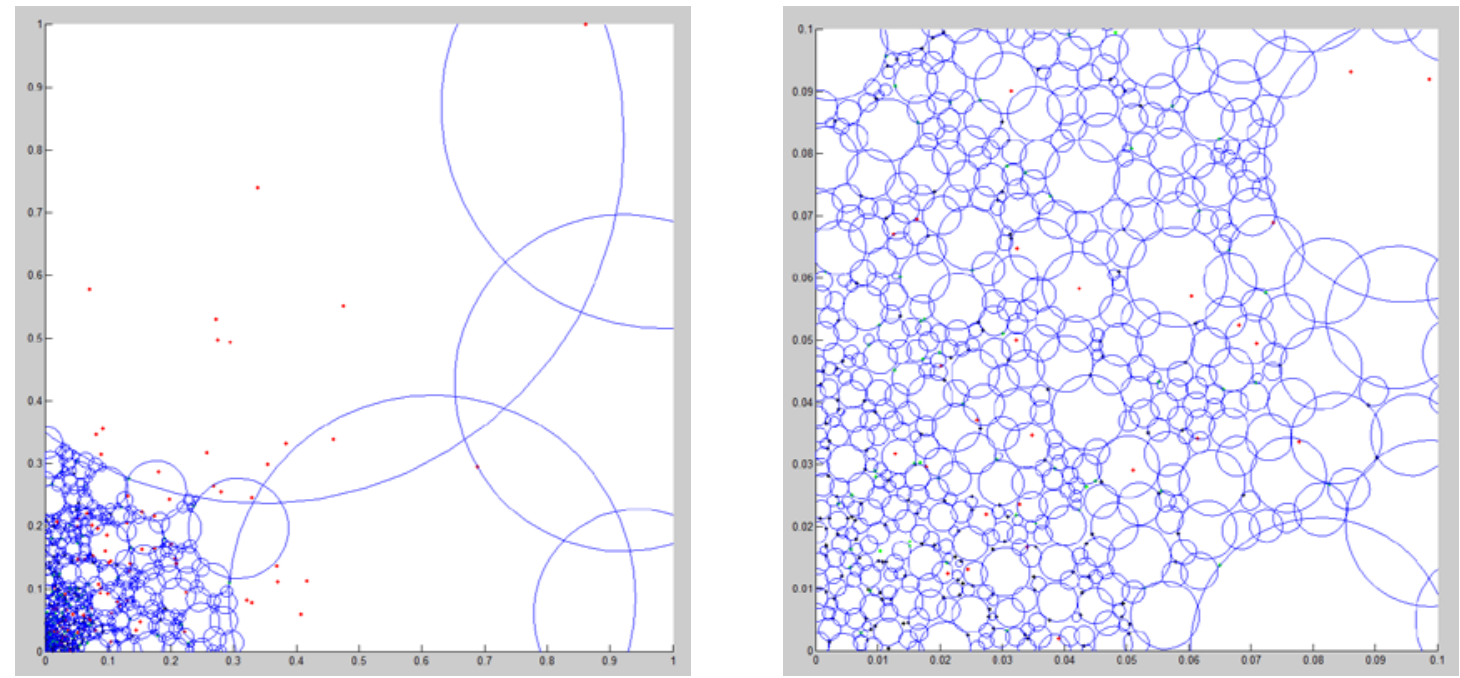

Figure A.9: AIS visualization of damage pattern 2, (b) zoomed in at [0 0.1] [0 0.1]
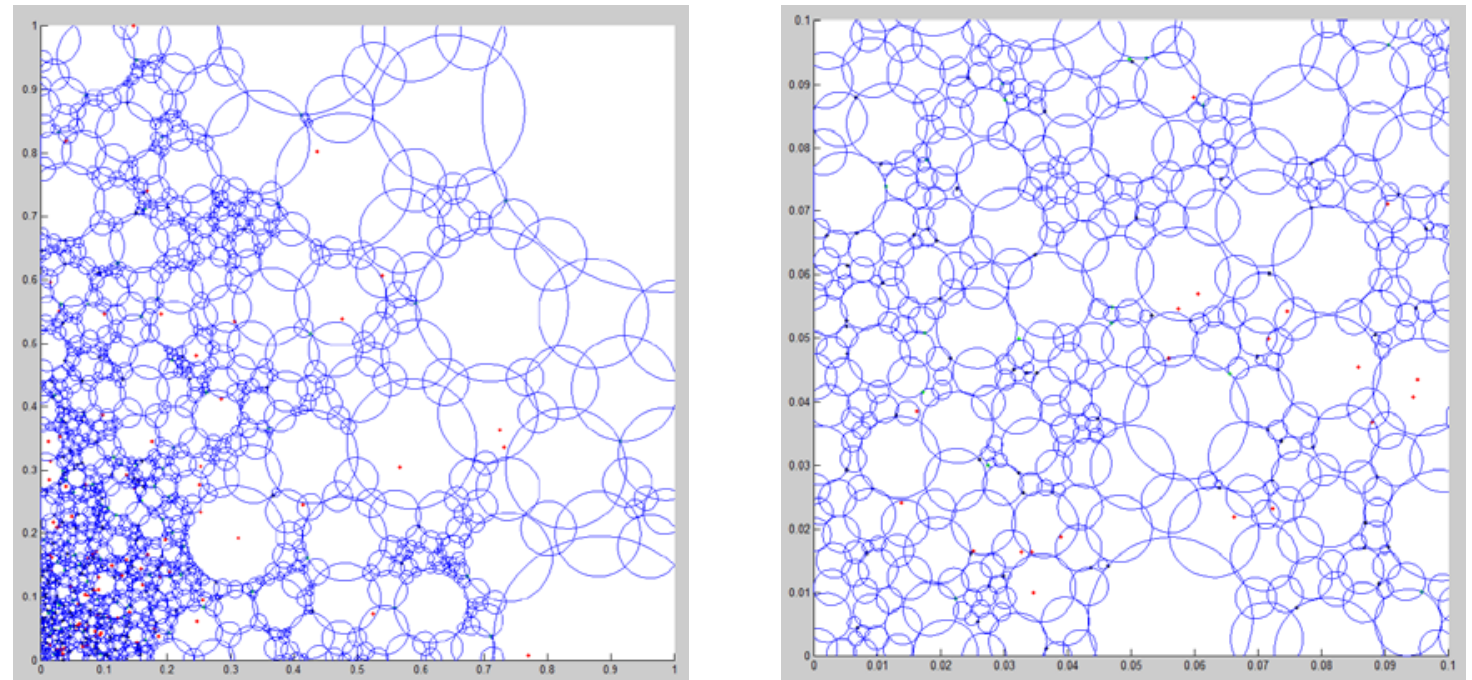

Figure A.10: AIS visualization of damage pattern 7, (b) zoomed in at [0 0.1] [0 0.1] 


\section{Appendix B: MATLAB Code Samples}

\section{Main workflow file}

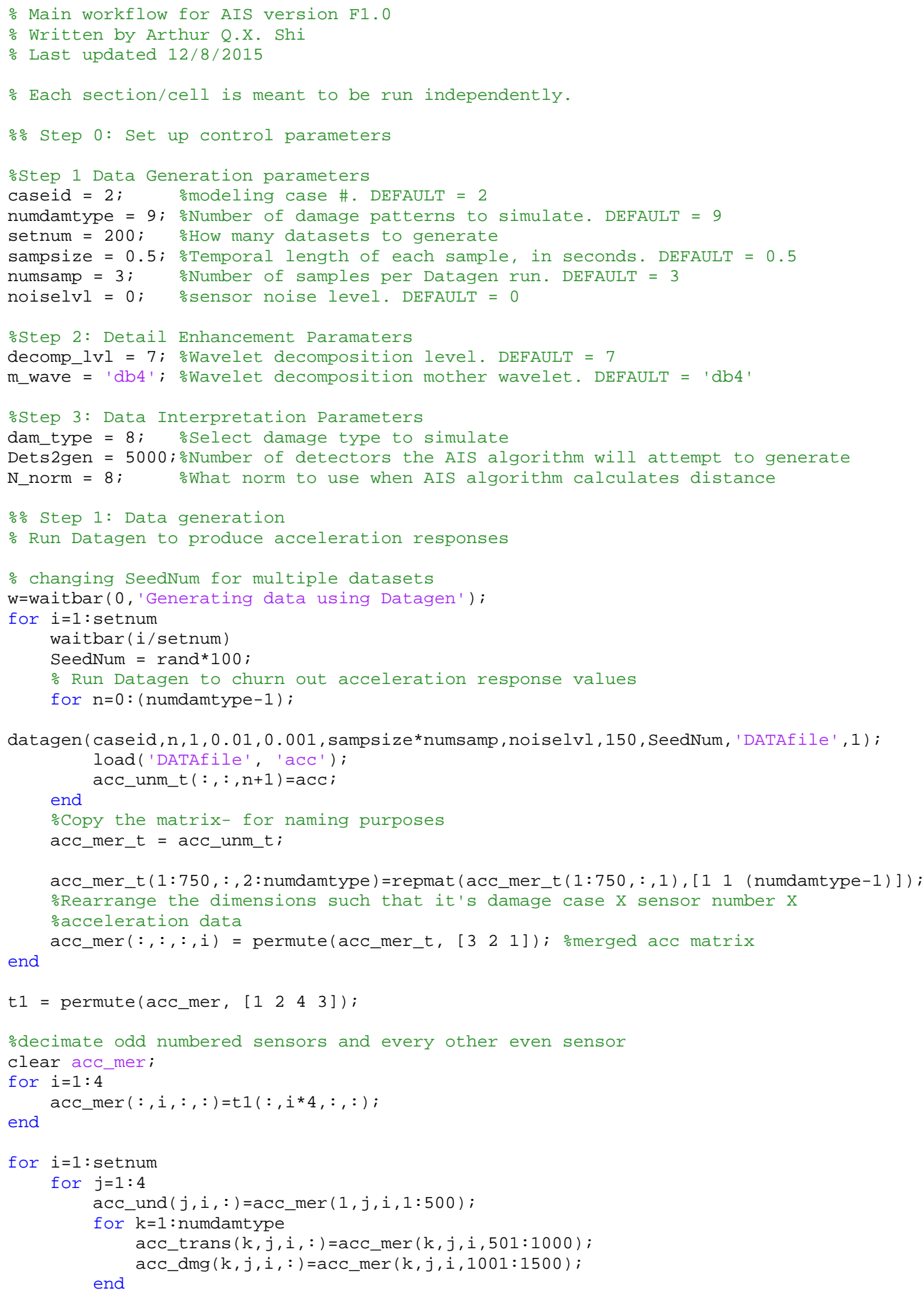


end

close (w)

\% Clear intermediary variables

clear n i j w t1 acc_unm_t acc_mer_t acc_unm acc

\%\% Step 2: Detail enhancement

$\%$ Run discrete wavelet transforms and filtering on acceleration response

\% Also perform energy calculations for target decomposition level

dwtmode('zpd'); \%Set zero padding as the edge padding option

$w=$ waitbar $(\Theta$, 'Calculating wavelet transforms...' $)$;

for $j=1$ : setnum \%Loop through all datasets

waitbar ( $j /$ setnum)

for $i=1: 4$ \%oop through all sensors

\%Calculate DWT for undamaged data

$[C, L]=\operatorname{wavedec}\left(\operatorname{acc} \_\right.$und $(i, j,:)$, decomp_lvl,m_wave $)$ \% \%avelet transform using

settings in step $\odot$

tmp $=\operatorname{appcoef}\left(C, L, m \_\right.$wave $) ;$\%Extract the approximation coefficients

cA7_undam $(i, j,:)=$ tmp; \%Store the coefficients in a matrix

\%Calculate total energy in sample (sum all coefficient energies)

$[E B]=$ wavedec $\left(\right.$ acc_und $(i, j,:), 1, m \_$wave $)$;

$\mathrm{E}=\mathrm{E} . \wedge 2$;

C_undam_E $(i, j)=\operatorname{sum}(E, 2)$;

$\mathrm{t} 1=0$;

\%Calculate energy of specific level

for $\mathrm{h}=1$ : size (cA7_undam, 3 )

end $\mathrm{t} 1=\mathrm{t} 1+\mathrm{cA} 7 \_$undam $(i, j, \mathrm{~h})^{\wedge} 2$;

CA7_undam_E $(i, j)=t 1$;

for $k=1$ :numdamtype \%Loop through all damage patterns (exception: undamaged, since that's only 1 pattern)

\%repeat process for center (transitioning/damaged) chunks

$[C, L]=$ wavedec $\left(\operatorname{acc} \_\operatorname{trans}(k, i, j,:)\right.$, decomp_lvl, m_wave $) ;$ \%Wavelet transform using settings in step $\odot$

tmp $=\operatorname{appcoef}\left(\mathrm{C}, \mathrm{L}, \mathrm{m} \_\right.$wave $) ;$\%xtract the approximation coefficients

cA7_trans $(k, i, j,:)=t m p ; \%$ Store the coefficients in a matrix

\%Calculate total energy in chunk

$[E B]=$ wavedec $\left(\right.$ acc_trans $\left.(k, i, j,:), 1, m \_w a v e\right)$;

$\mathrm{E}=\mathrm{E} \cdot \wedge 2$;

C_trans_E $(k, i, j)=\operatorname{sum}(E, 2)$;

\%repeat process for right (damaged) chunks

$[C, L]=$ wavedec $\left(\operatorname{acc} \_d m g(k, i, j,:)\right.$, decomp_lvl, m_wave $)$; \%Wavelet transform using

settings in step 0

tmp $=\operatorname{appcoef}\left(\mathrm{C}, \mathrm{L}, \mathrm{m} \_\right.$wave $) ;$\%xtract the approximation coefficients

cA7_dam $(k, i, j,:)=$ tmp; \%Store the coefficients in a matrix

\%Calculate total energy in chunk

$\left[E B^{\prime}\right]=$ wavedec $\left(\operatorname{acc} \_d m g(k, i, j,:), 1, m \_\right.$wave $)$;

$\mathrm{E}=\mathrm{E} . \wedge 2$;

C_dam_E $(k, i, j)=\operatorname{sum}(E, 2)$;

\%Calculate energy for the coefficients at the individual level and store them

t2 $=0 ;$

$\mathrm{t} 3=0$;

for $\mathrm{h}=1$ : size (cA7_trans, 4)

$\mathrm{t} 2=\mathrm{t} 2+\mathrm{cA} 7 \mathrm{trans}(\mathrm{k}, \mathrm{i}, \mathrm{j}, \mathrm{h}) \wedge 2$

end t3=t3+cA7_dam $(k, i, j, h)^{\wedge} 2$;

CA7_trans_E $(k, i, j)=t 2$;

cA7_dam_E $(k, i, j)=\mathrm{t} 3$; 


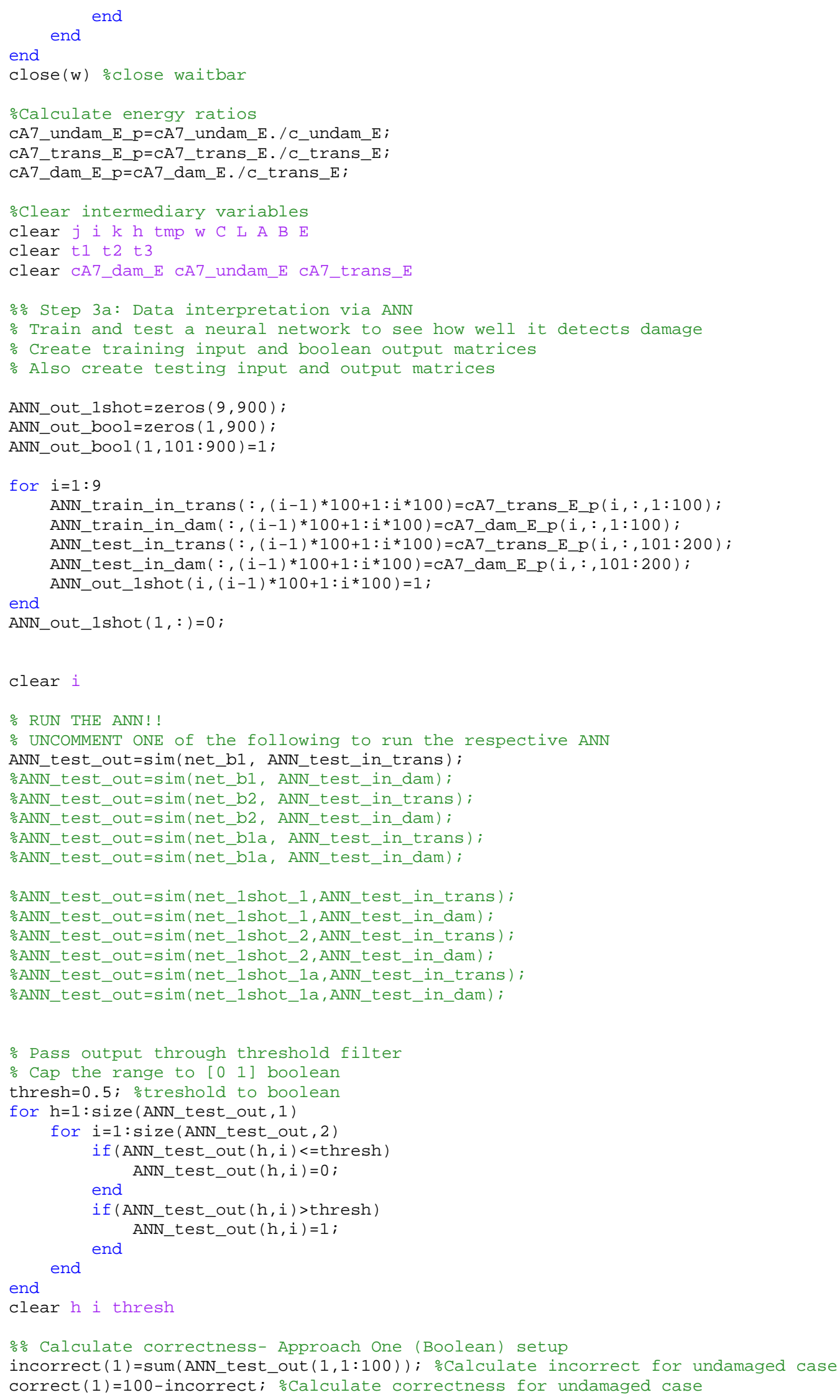




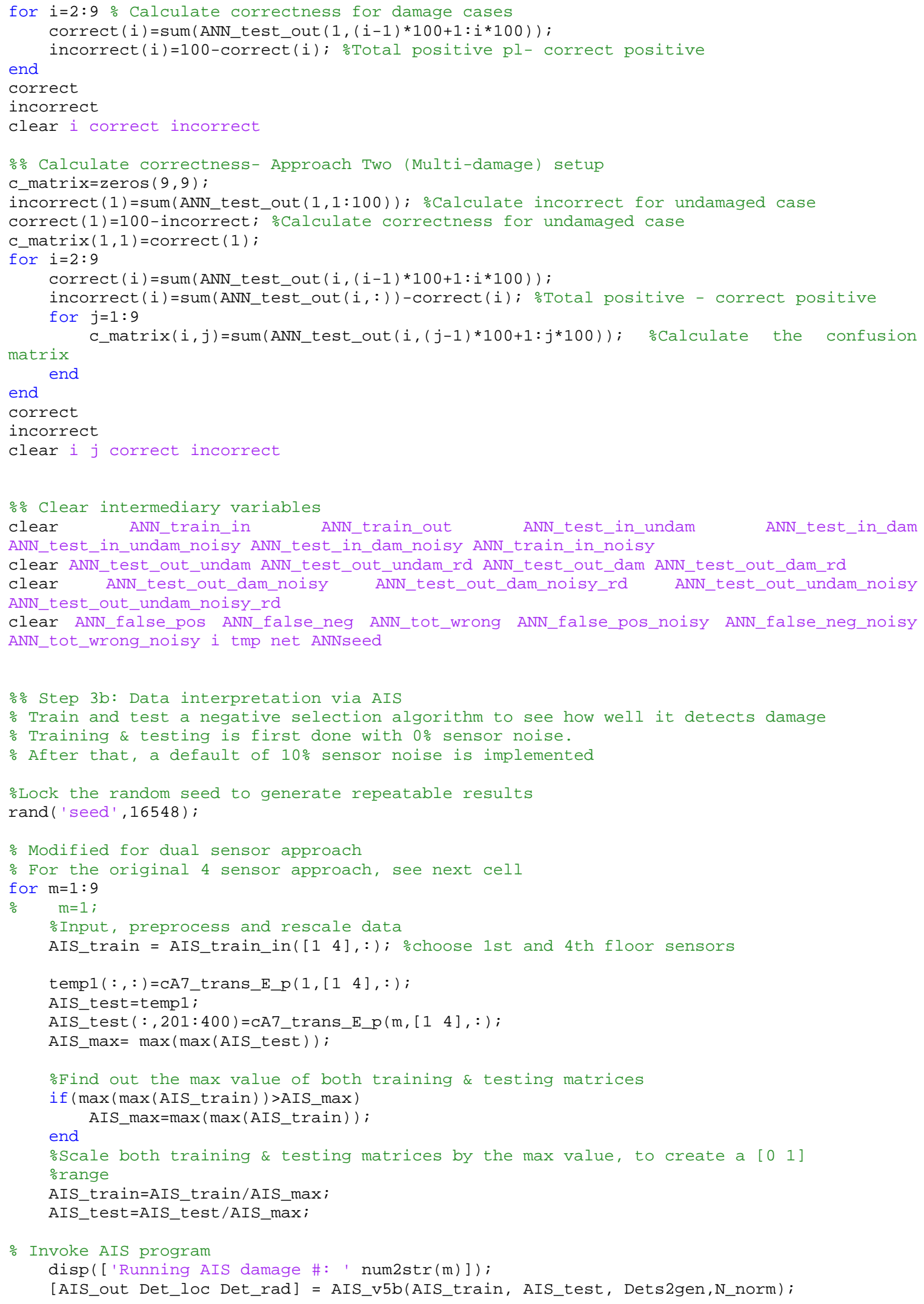


\% Calculate effectiveness metrics- cA7 matrix version

AIS_mat = AIS_out; \%point to AIS output matrix

$\operatorname{self}(1)=\operatorname{sum}($ AIS_mat $(1: 200,:))$;

nonself $(1)=200-\operatorname{self}(1)$

$\operatorname{self}(2)=\operatorname{sum}($ AIS_mat $(201: 400,:))$;

nonself $(2)=200-\operatorname{self}(2)$;

self

nonself

end \%end loop here if non-visualizing

$\%$ visualize training \& testing data

figure

hold on

$\operatorname{axis}\left(\left[\begin{array}{llll}0 & 1 & 0 & 1\end{array}\right]\right)$;

for $i=1$ : size (AIS_train, 2) end

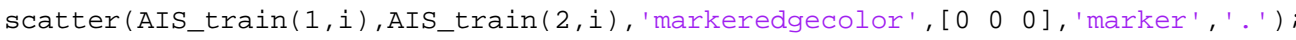

for $i=1: 200$ end scatter(AIS_test(1,i),AIS_test(2,i), 'markeredgecolor', [0 $\left.\begin{array}{lll}0 & 0\end{array}\right]$, 'marker' , '. ');

for $i=201: 400$

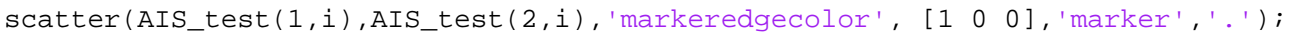
end

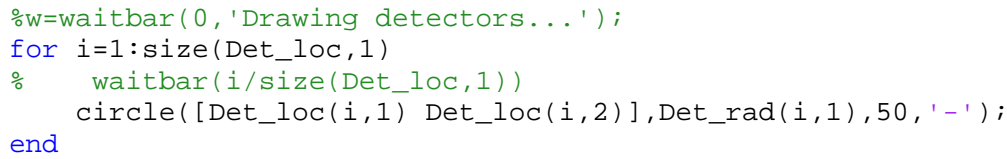




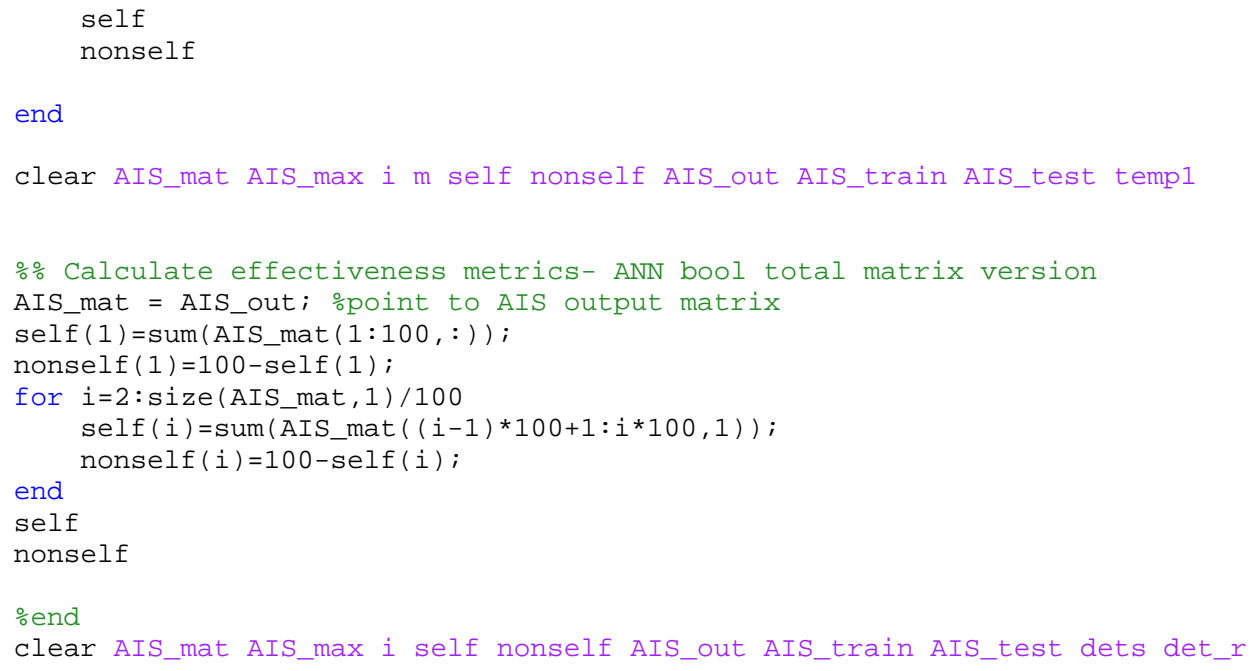




\section{AIS code:}

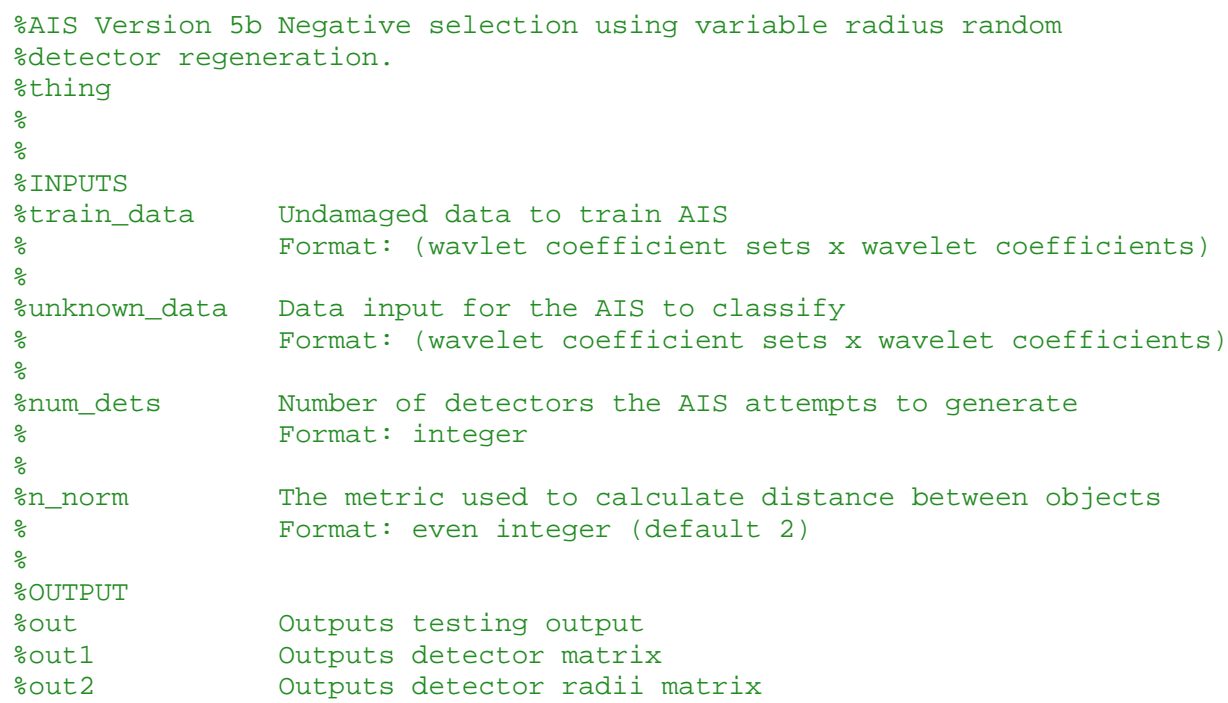




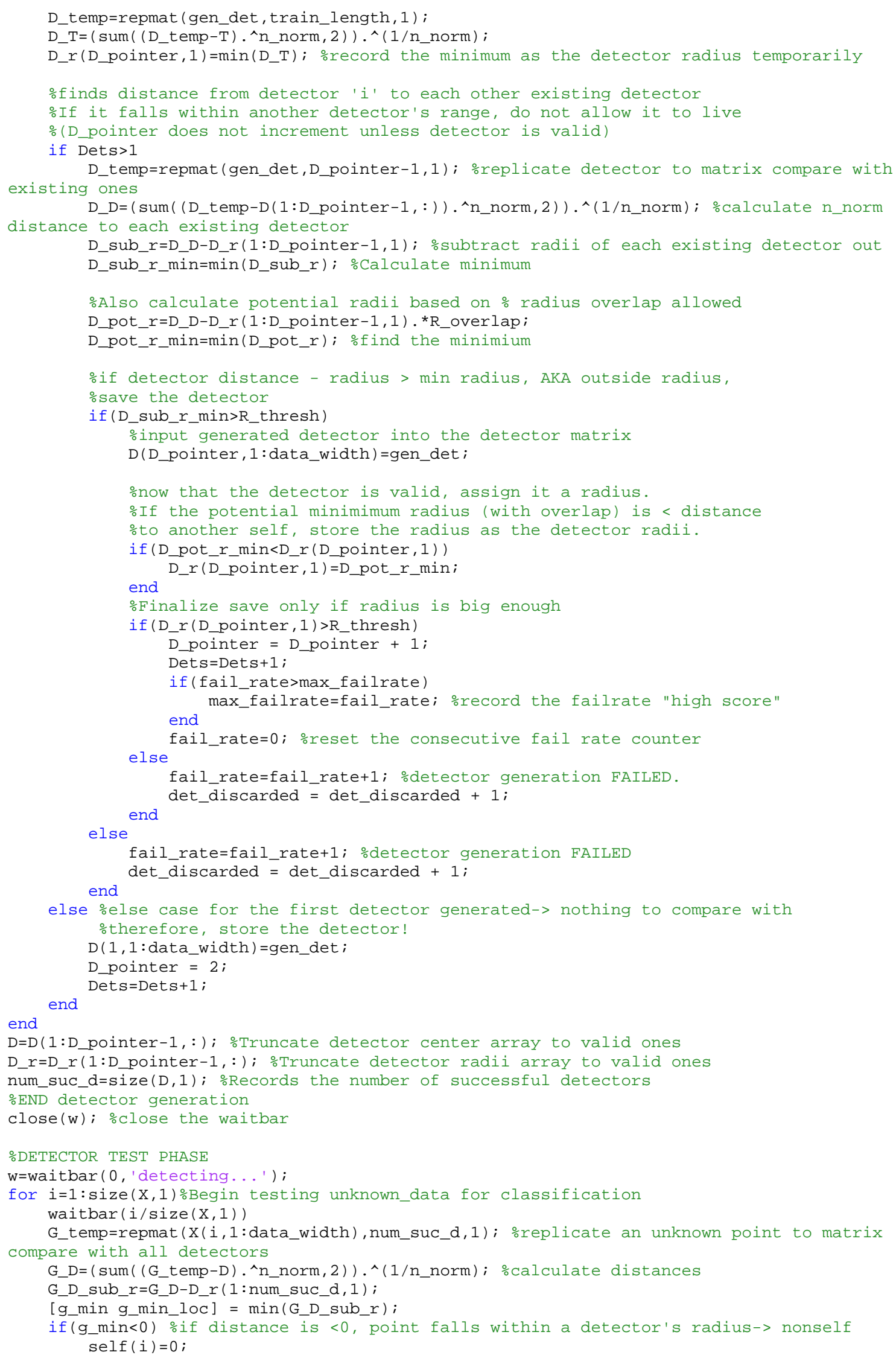




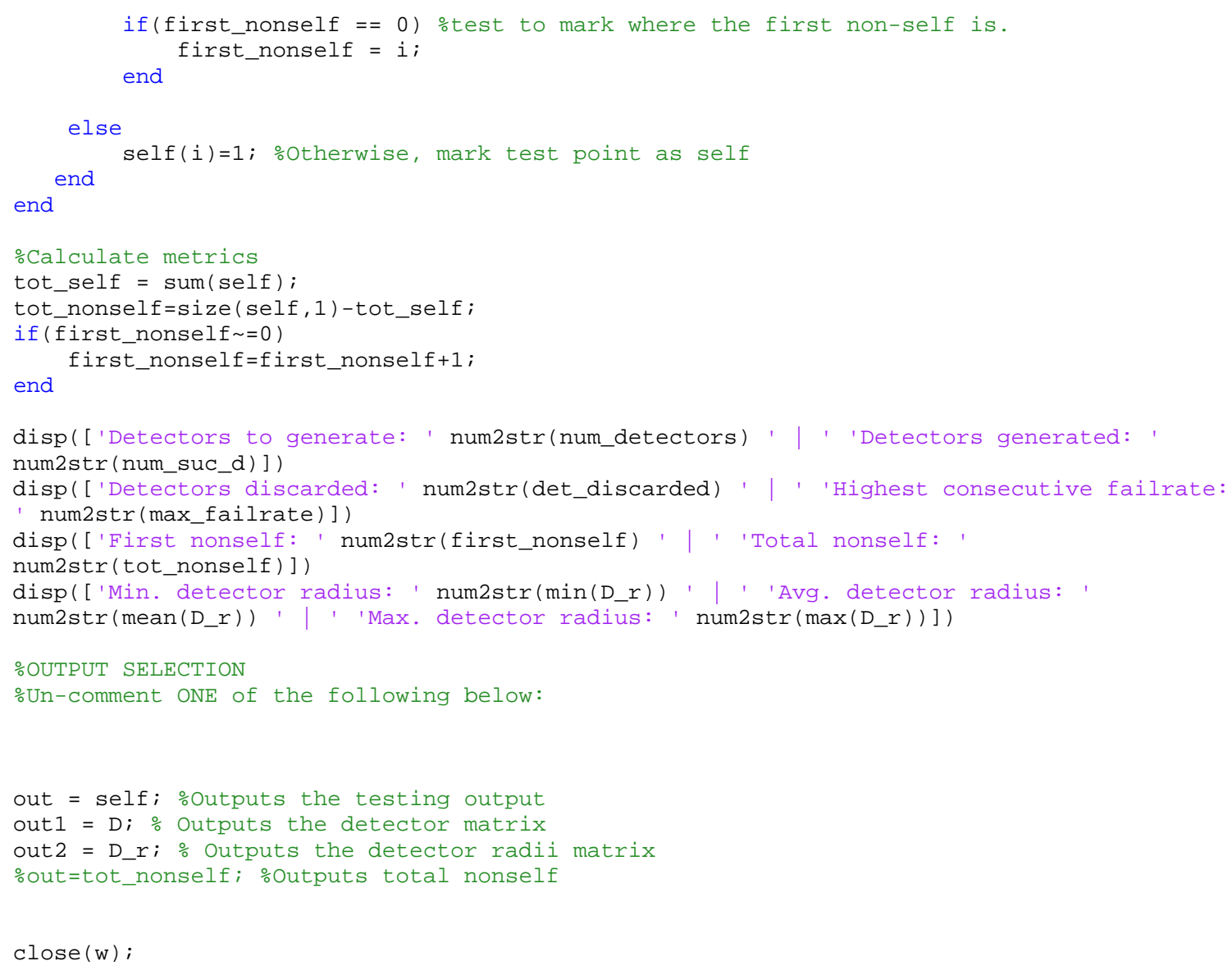

